\title{
Coerência parcial e aplicações
}

\author{
Kim Samejima Mascarenhas Lopes
}

\author{
DissERTAÇÃO APRESENTADA \\ $\mathrm{AO}$ \\ Instituto de Matemática e Estatística \\ DA \\ Universidade DE SÃo PaUlo \\ PARA \\ OBTENÇÃO DO TÍTULO \\ $\mathrm{DE}$ \\ Mestre EM CiÊnCIAS
}

Programa: Estatística

Orientador: Prof. Dr. Pedro Alberto Morettin

São Paulo, Abril de 2009 


\title{
Coerência Parcial e Aplicações
}

\author{
Este exemplar corresponde à redação \\ final da dissertação devidamente corrigida \\ e defendida por Kim Samejima Mascarenhas Lopes \\ e aprovada pela Comissão Julgadora.
}

São Paulo, 24 de Abril de 2009.

Banca Examinadora:

- Prof. Dr. Pedro Alberto Morettin (orientador) - IME-USP

- Prof. Dra. Clélia Maria de Castro Toloi - IME-USP

- Prof. Dr. João Ricardo Sato - Universidade Federal do ABC 


\section{Agradecimentos}

Agradeço, antes de tudo, a Deus, por tudo o que Ele tem feito em minha vida. Agradeço ao meu orientador, Pedro, por toda a compreensão, paciência e atenção que teve por mim durante o desenvolvimento deste trabalho. Agradeço a minha esposa, Melyssa, e peço desculpas por todos os momentos em que tive que me ausentar para estudar. Agradeço ao colega e amigo João Ricardo Sato, que gentilmente cedeu dados de seu próprio eletroencefalograma (EEG) e me auxiliou na aplicação dos conhecimentos nos bancos de dados. Finalmente, agradeço a minha mãe, por todo o sacrifício e dedicação que teve à minha educação, sem a qual não seria possível a conclusão ou mesmo início deste trabalho. 


\section{Resumo}

Neste trabalho foram estudadas algumas formas de relação entre séries temporais multivariadas. Discutiu-se, inicialmente, a função de coerência, uma função análoga a função de correlação(que é dada no domínio do tempo) calculada no domínio da freqüência. Foram estudadas também as funções de coerência parcial e coerência parcial direcionada. A função de coerência parcial mede a relação entre duas componentes de uma série multivariada, isolados os efeitos de outra série. Em linhas gerais, a Coerência Parcial Direcionada pode ser interpredata como a decomposição da coerência parcial a partir de modelos autoregressivos multivariados. Esse conceito pode ser interpretado como uma representação do conceito de causalidade de Granger no domínio da freqüência. Finalmente, foram aplicadas as funções acima em dois conjuntos de dados: um modelo VAR(1) trivariado simulado e dados de medições de eletroencefalograma.

Palavras-chave: Séries Temporais, Espectro cruzado, Coerência, Coerência parcial, Coerência Parcial direcionada. 


\section{Abstract}

In this work we studied relationships between multivariate time series. We discussed the coherence function, a function similar to the correlation function(calculated in time domain) in frequency domain. Next, we discussed partial coherence and partial directed coherence. The partial coherence measures the relationship between two components of a multivariate time series, after removing the influence of another time series. Generally, the partial directed coherence can be interpreted as the decompositioin of the partial coherence from multivariate autoregressive models. We can interpret this function as a representation of the Granger causality concept in frequency domain. Finally, we applied these concepts in two situations: a simulated VAR(1) model and an electroencefalogram database.

Keywords: Time series, Cross Spectrum, Coherence, Partial Coherence, Partial Directed Coherence. 


\section{Sumário}

1 Introdução 1

2 Conceitos Iniciais 2

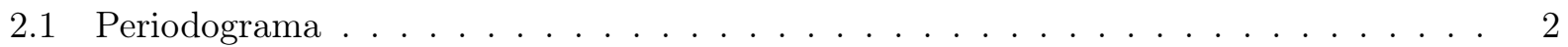

2.2 Função de Covariância Cruzada . . . . . . . . . . . . . . . . . . . 3

2.3 O Espectro Cruzado . . . . . . . . . . . . . . . . . . . . . 4

2.3.1 Estimação do Espectro Cruzado . . . . . . . . . . . . . . . . . . . 4

2.3.2 Distribuição do Periodograma Cruzado Suavizado . . . . . . . . . . . . . . 5

2.4 Coespectro e Espectro de Quadratura . . . . . . . . . . . . . . . . . 5

2.5 Função de Coerência . . . . . . . . . . . . . . . . . . . . . . 6

2.6 Estimação da Coerência . . . . . . . . . . . . . . . . . . . 7

2.6 .1 Distribuição assintótica de $\kappa_{X Y}^{2}(\omega) \ldots \ldots \ldots \ldots \ldots$

2.7 Considerações acerca da Coerência via Análie de Fourier . . . . . . . . . . . . . . 8

3 Coerência Parcial $\quad 9$

3.1 Filtro de Regressão . . . . . . . . . . . . . . . . . . . . . . . . . . . . . . . . 9

3.2 Função de Coerência Parcial . . . . . . . . . . . . . . . . . . . . . . . 12

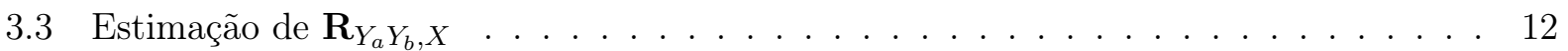

3.4 Distribuição assintótica de $\mathbf{R}_{Y_{a} Y_{b}, X}^{(T)} \ldots \ldots \ldots \ldots \ldots \ldots \ldots$

4 Coerência Parcial Direcionada $\quad 15$

4.1 Coerência Direcionada - Primeiros Conceitos . . . . . . . . . . . . . . . . . 15

4.2 Causalidade de Granger . . . . . . . . . . . . . . . . . . . . 16

4.3 Coerência Parcial Direcionada . . . . . . . . . . . . . . . . . . . . 17

4.4 Distribuição Assintótica . . . . . . . . . . . . . . . . . . . 18

5 Aplicações $\quad 20$

5.1 Resultados a partir de simulações . . . . . . . . . . . . . . . . . . . 20

5.2 Resultados nos dados de EEG . . . . . . . . . . . . . . . . . 25

5.3 Discussão . . . . . . . . . . . . . . . . . . . . . . . . . 34

6 Considerações Finais $\quad 38$ 
A Sintaxe R - Funções utilizadas $\quad 39$

B Gráficos e Figuras de Coerência Parcial e PDC 44

$\begin{array}{ll}\text { Referências Bibliográficas } & 57\end{array}$ 


\section{Capítulo 1}

\section{Introdução}

Dentre os assuntos mais interessantes da estatística aplicada, a relação entre variáveis é uma das mais estudadas. Técnicas de análise de regressão, análise de variância e análise multivariada são amplamente discutidas na literatura. Em séries temporais, particularmente, destacam-se as discussões sobre de modelos de séries temporais multivariados, modelos de cointegração e causalidade. As aplicações são das mais diversas, partindo desde a área médica até a ramos de finanças e economia.

Neste texto, discutiremos a definição e propriedades da função de coerência. Coerência é uma medida de relação entre séries temporais análoga à função de correlação de Pearson, porém, calculada no domínio da freqüência. Discutiremos inicialmente, conceitos essenciais para a definição de tal função.

Isto posto, estudaremos o problema de sua estimação e construção de intervalos de confiança a partir de sua distribuição assintótica. Apresentaremos a forma usual de cálculo da função de coerência, construída a partir da transformada discreta de Fourier da função de covariância cruzada.

No capítulo 2, apresentaremos os primeiros conceitos necessários para o estudo da coerência parcial. Discutiremos o espectro, o espectro cruzado e definiremos a Função de Coerência via transformada de Fourier e estudaremos seus estimadores, distribuições assintóticas e intervalos de confiança.

Discutiremos no capítulo 3, a Coerência Parcial. Sua definição parte da função de coerência calculada a partir dos resíduos de um filtro linear que elimina a correlação com outras séries. Veremos as peculiaridades desta função em relação à função de coerência.

No capítulo 4, partiremos das definições apresentadas por Baccalá e Sameshima (2001) para apresentar o conceito de Coerência Parcial Direcionada.

Finalmente, no capítulo 5, apresentaremos aplicações das funções de coerência estudadas ao longo deste texto. 


\section{Capítulo 2}

\section{Conceitos Iniciais}

Iniciamos nossos estudos apresentando as técnicas usuais de cálculo da coerência através da transformada de Fourier. Para tanto, introduziremos conceitos tais como covariância cruzada, coespectro e espectro de quadratura. Tais definições nos ajudarão na construção da função de coerência e no estudo de suas propriedades.

\subsection{Periodograma}

Seja $\left\{X_{t}\right\}$ série temporal discreta, fracamente estacionária, com função de autocovariância $\gamma_{X X}(u)=\operatorname{cov}\{X(t+u), X(t)\}, t, u \in \mathbb{Z}$.

Definição 1 (Espectro) Supondo,

$$
\sum_{u=-\infty}^{\infty}\left|\gamma_{X X}(u)\right|<\infty
$$

definimos a função densidade espectral, ou simplesmente espectro, de $\left\{X_{t}\right\}$ como:

$$
f_{X X}(\omega)=\frac{1}{2 \pi} \sum_{u=-\infty}^{\infty} \gamma_{X X}(u) e^{-i \omega u},-\pi<\omega<\pi .
$$

Segue-se que $f_{X X}(\omega)$ é real (porque $\gamma_{X X}(u)$ é par), periódico de período $2 \pi$ e uniformemente contínuo.

Definição 2 (Periodograma) Seja $\left\{X_{t}\right\}$ série temporal discreta com espectro $f_{X X}(\omega)$. Considere que temos observados $\left\{X_{0}, \ldots, X_{T-1}\right\}$. Calculemos então a quantidade:

$$
d_{X}^{(T)}(\omega)=\sum_{u=0}^{T-1} X(u) e^{-i \omega u}
$$


chamada Transformada de Fourier Discreta. O periodograma de $\left\{X_{0}, \ldots, X_{T-1}\right\}$ é definido por

$$
\begin{aligned}
I_{X X}^{(T)}(\omega) & :=\frac{1}{2 \pi T}\left|d_{X}^{(T)}(\omega)\right|^{2} \\
& =\frac{1}{2 \pi T}\left|\sum_{u=0}^{T-1} X(u) e^{-i \omega u}\right|^{2} .
\end{aligned}
$$

Note que podemos escrever $\left|d_{X}^{(T)}(\omega)\right|^{2} \operatorname{como} d_{X}^{(T)}(\omega) \times \overline{d_{X}^{(T)}(\omega)}$, onde $\overline{d_{X}^{(T)}(\omega)}$ representa o complexo conjugado de $d_{X}^{(T)}(\omega)$. Dessa forma, (2.3) fica

$$
I_{X X}^{(T)}(\omega)=(2 \pi T)^{-1} d_{X}^{(T)}(\omega) \times \overline{d_{X}^{(T)}(\omega)} .
$$

Vale ressalvar que $I_{X X}^{(T)}(\omega)$ é um estimador de $f_{X X}(\omega)$, mas tem propriedades não muito boas. Por exemplo, sua variância é constante independentemente do tamanho da amostra, sendo portanto um estimador inconsistente. Melhores estimadores podem ser construídos com a suavização de $I_{X X}^{(T)}(\omega)$. Veja, por exemplo, Morettin e Toloi (2006) ou Shumway e Stoffer (2006).

Dessa forma, considere $\mathrm{s}(\mathrm{T})$ um inteiro tal que $2 \pi s(T) / T$ seja próximo de $\omega$. Brillinger (2001) mostra que os $(2 \mathrm{~m}+1)$ valores de $I_{X X}^{(T)}($ dado em $(2.3)), I_{X X}^{(T)}(2 \pi[s(T)+j] / T), j=0, \pm 1, \pm 2, \ldots, \pm m$, são aproximadamente independentes. Dessa forma, a partir destes valores pode-se produzir estimativas aproximadamente independentes de $f_{X X}$, o que sugere um estimador da forma

$$
f_{X X}^{(T)}(\omega)=m^{-1} \sum_{j=1}^{m} I_{X X}^{(T)}\left(\omega+\frac{2 \pi j}{T}\right)
$$

se $\omega=0, \pm 2 \pi, \pm 4 \pi, \ldots$ ou se $\omega=0, \pm \pi, \pm 3 \pi, \ldots$ e T é par e

$$
f_{X X}^{(T)}(\omega)=m^{-1} \sum_{j=1}^{m} I_{X X}^{(T)}\left(\omega-\frac{\pi}{T}+\frac{2 \pi j}{T}\right)
$$

com $\omega= \pm \pi, \pm 3 \pi, \ldots$ e $\mathrm{T}$ é ímpar. Esta estimativa de $f_{X X}(\omega)$ é chamada de Periodograma Suavizado. A partir de tal suavização, consegue-se garantir a conscistência do estimador do periodograma. As estimativas (2.5) e (2.6) são mais facilmente calculadas via FFT(Fast Fourier Transform) do que o caso em que temos uma única expressão para o estimador (veja Brillinger, 2001).

\subsection{Função de Covariância Cruzada}

Suponha que o vetor $\left(X_{t}, Y_{t}\right)^{\prime}$ fracamente estacionário (como descrito em Brillinger, 2001)). Sejam

$$
\begin{aligned}
& \mathbf{E}\left(X_{t}\right)=\mu_{X}, \\
& \mathbf{E}\left(Y_{t}\right)=\mu_{Y},
\end{aligned}
$$




$$
\begin{gathered}
\operatorname{cov}\left(X_{t}, X_{t+k}\right)=\gamma_{X X}(k), \\
\operatorname{cov}\left(Y_{t}, Y_{t+k}\right)=\gamma_{Y Y}(k), \\
\operatorname{cov}\left(X_{t}, Y_{t+k}\right)=\gamma_{X Y}(k),
\end{gathered}
$$

onde a Função de Covariância Cruzada $\gamma_{X Y}(k)$ é definida por:

$$
\gamma_{X Y}(k)=\operatorname{cov}\left(X_{t}, Y_{t+k}\right), k=0, \pm 1, \pm 2, \ldots
$$

É interessante notar que a função (2.7) não é par. Neste sentido, temos que

$$
\gamma_{X Y}(k) \neq \gamma_{X Y}(-k)
$$

mas

$$
\gamma_{X Y}(k)=\gamma_{Y X}(-k)
$$

\subsection{O Espectro Cruzado}

Analogamente à função densidade espectral, definimos o Espectro Cruzado(ou função densidade espectral cruzada) como a transformada de Fourier da função de covariância cruzada(2.7). Assim, supondo-se $\sum_{k}\left|\gamma_{X Y}(k)\right|<\infty$, define-se

$$
f_{X Y}(\omega)=\frac{1}{2 \pi} \sum_{k=-\infty}^{\infty} \gamma_{X Y}(k) e^{-i \omega k},-\pi<\omega<\pi
$$

Note que:

- Por conta de $\gamma_{X Y}($.$) ser uma função não par, f_{X Y}($.$) é uma função complexa;$

- Pela definição acima, temos que $f_{X Y}(\omega)=f_{Y X}(-\omega)=\overline{f_{X Y}(-\omega)}$.

\subsubsection{Estimação do Espectro Cruzado}

Assim como para a função densidade espectral, podemos construir estimadores da função densidade espectral cruzada. Considere a função $d_{X}^{(T)}$ descrita em (2.2). Analogamente, defina $d_{Y}^{(T)}$. Pode-se inicialmete considerar a seguinte estimativa do espectro cruzado, denominada Periodograma Cruzado:

$$
I_{X Y}^{(T)}(\omega)=(2 \pi T)^{-1} d_{X}^{(T)}(\omega) \times \overline{d_{Y}^{(T)}(\omega)} .
$$

Novamente, como o periodograma definido na definição 2, as propriedades do periodograma cruzado são insatisfatórias para considerá-lo um estimador consistente de $f_{X Y}$. Portanto, consideraremos o periodograma cruzado suavizado, dado a seguir:

$$
\left.f_{X Y}^{(T)}(\omega)=(2 m+1)^{-1} \sum_{j=-m}^{m} I_{X Y}^{(T)}\left(\frac{2 \pi[s(T)+j}{T}\right]\right)
$$

onde $\mathrm{s}(\mathrm{T})$ é um inteiro. 


\subsubsection{Distribuição do Periodograma Cruzado Suavizado}

Brillinger(2001) mostra que o espectro cruzado estimado dado em (2.12) possui matriz de covariância aproximadamente dada por

$$
\begin{gathered}
\operatorname{cov}\left(f_{X Y}^{(T)}(\omega), f_{X Y}^{(T)}(\lambda)\right)= \\
\frac{\nu\{\omega-\lambda\} f_{X X}(\omega) f_{Y Y}(-\omega)+\nu\{\omega+\lambda\} f_{X Y}(\omega) f_{Y X}(-\omega)}{2 m+1}+O\left(T^{-1}\right)
\end{gathered}
$$

se $\omega \neq 0(\bmod \pi)$ e

$$
\begin{gathered}
\operatorname{cov}\left(f_{X Y}^{(T)}(\omega), f_{X Y}^{(T)}(\lambda)\right)= \\
\frac{\nu\{\omega-\lambda\} f_{X X}(\omega) f_{Y Y}(-\omega)+\nu\{\omega+\lambda\} f_{X Y}(\omega) f_{Y X}(-\omega)}{2 m}+O\left(T^{-1}\right)
\end{gathered}
$$

se $\omega \equiv 0(\bmod \pi),-\infty<\omega, \lambda<\infty$ e $\nu\{$.$\} é a extensão 2 \pi$-periódica do núcleo de Kronecker, definido por

$$
\delta\{\omega\}=\left\{\begin{array}{l}
1, \omega=0 \\
0, \text { c.c. }
\end{array}\right.
$$

\subsection{Coespectro e Espectro de Quadratura}

Dado que o espectro cruzado é uma função complexa, pode-se decompô-lo em duas partes, uma real e uma imaginária:

$$
\begin{aligned}
f_{X Y}(\omega) & =\frac{1}{2 \pi}\left[\sum_{k} \gamma_{X Y}(k) e^{-i \omega k}\right] \\
& =\frac{1}{2 \pi}\left[\sum_{k} \gamma_{X Y}(k)(\cos (\omega k)-i \sin (\omega k))\right] \\
& =\frac{1}{2 \pi}\left[\sum_{k} \gamma_{X Y}(k) \cos (\omega k)-i \sum_{k}\left(\gamma_{X Y}(k) \sin (\omega k)\right]\right. \\
& =\frac{1}{2 \pi} \sum_{k} \gamma_{X Y}(k) \cos (\omega k)-i \frac{1}{2 \pi} \sum_{k} \gamma_{X Y}(k) \sin (\omega k)= \\
& =c_{X Y}(\omega)-i q_{X Y}(\omega)
\end{aligned}
$$

Dizemos que $c_{X Y}(\omega)$ é o coespectro e $q_{X Y}(\omega)$ é o espetro de quadratura. Podemos ainda reescrever $c_{X Y}($.$) e q_{X Y}($.$) da seguinte maneira:$ 
- Coespectro:

$$
\begin{aligned}
c_{X Y}(\omega) & =\frac{1}{2 \pi} \sum_{k} \gamma_{X Y}(k) \cos (\omega k) \\
& =\frac{1}{2 \pi}\left\{\gamma_{X Y}(0)+\sum_{k=1}^{\infty}\left[\gamma_{X Y}(k)+\gamma_{Y X}(k)\right] \cos (\omega k)\right\}
\end{aligned}
$$

\section{- Espectro de Quadratura:}

$$
\begin{aligned}
q_{X Y}(\omega) & =\frac{1}{2 \pi} \sum_{k} \gamma_{X Y}(k) \sin (\omega k) \\
& =\frac{1}{2 \pi}\left\{\sum_{k=1}^{\infty}\left[\gamma_{X Y}(k)-\gamma_{Y X}(k)\right] \sin (\omega k)\right\}
\end{aligned}
$$

De uma maneira geral, o Coespectro mede o quanto oscilam as duas séries com a mesma fase (ou com sinais opostos, com um shift de fases $1 / 2$ ciclo). O Espectro de Quadratura mede o quanto as séries tendem a oscilar com fases diferindo de $1 / 4$ de ciclo em qualquer direção.

Uma forma alternativa de definir o Espectro Cruzado é dada por

$$
f_{X Y}(\omega)=\alpha_{X Y} e^{i \phi_{X Y}(\omega)}
$$

onde:

$$
\alpha_{X Y}=\sqrt{c_{X Y}^{2}(\omega)+q_{X Y}^{2}(\omega)}
$$

e

$$
\phi_{X Y}(\omega)=\tan ^{-1}\left[\frac{-q_{X Y}(\omega)}{c_{X Y}(\omega)}\right]
$$

\subsection{Função de Coerência}

Finalmente, definimos a Função de Coerência (ou coerência quadrática), como

$$
\kappa_{X Y}^{2}(\omega)=\frac{\left|f_{X Y}(\omega)\right|^{2}}{f_{X X}(\omega) f_{Y Y}(\omega)}
$$

A coerência mede o quadrado da correlação linear entre dois componentes de um processo bivariado na freqüência $(\omega)$ e é análogo ao quadrado do coeficiente de correlação de Pearson, no domínio da freqüência.

Como temos que

$$
\left|f_{X Y}(\omega)\right|^{2} \leq f_{X X}(\omega) \cdot f_{Y Y}(\omega)
$$


obtemos

$$
0 \leq \kappa_{X Y}^{2}(\omega) \leq 1
$$

\subsection{Estimação da Coerência}

Considerando as definições 1 e 2, então temos que um estimador para a coerência pode ser dado como segue. Assim como na estimação do espectro e do espectro cruzado, o uso de um estimador baseado no periodograma não é recomendado, uma vez que a coerência teria valor constante igual a 1. Dessa forma, desde que as quantidades $f_{X X}^{(T)}(\omega), f_{Y Y}^{(T)}(\omega)$ e $f_{X Y}^{(T)}(\omega)$ (respectivamente os periodogramas das séries $\left\{X_{t}\right\}$ e $\left\{Y_{t}\right\}$ e o periodograma cruzado entre as séries $\left\{X_{t}\right\}$ e $\left\{Y_{t}\right\}$ ) estejam bem definidas e sejam não nulos, então a razão

$$
\widehat{\kappa_{X Y}^{2}(\omega)}=\frac{\left|f_{X Y}^{(T)}(\omega)\right|^{2}}{f_{X X}^{(T)}(\omega) f_{Y Y}^{(T)}(\omega)}
$$

é um estimador para $\kappa_{X Y}^{2}(\omega)$ na freqüência $\omega$.

2.6.1 Distribuição assintótica de $\widehat{\kappa_{X Y}^{2}(\omega)}$

Considerando que

$$
\begin{aligned}
\widehat{\kappa_{X Y}^{2}(\omega)} & =\frac{\left|f_{X Y}^{(T)}(\omega)\right|^{2}}{f_{X X}^{(T)}(\omega) f_{Y Y}^{(T)}(\omega)} \Rightarrow \widehat{\kappa_{X Y}(\omega)}=\frac{\left|f_{X Y}^{(T)}(\omega)\right|}{\sqrt{f_{X X}^{(T)}(\omega) f_{Y Y}^{(T)}(\omega)}} \\
& \approx \kappa_{X Y}(\omega)+\frac{a \cdot c_{X Y}(\omega)+b \cdot q_{X Y}(\omega)}{\kappa_{X Y}(\omega) f_{X X}(\omega) f_{Y Y}(\omega)}-\frac{1}{2} \kappa_{X Y}(\omega)\left[\frac{c}{f_{X X}(\omega)}+\frac{d}{f_{Y Y}(\omega)}\right]
\end{aligned}
$$

com $a, b, c$ e $d$ constantes reais e $\mathrm{f}(),. \mathrm{c}($.$) e \mathrm{q}($.$) definidos como antes(veja a definição 1$, equações $(2.14)$ e $(2.15))$.

Mas em Brillinger(2001) temos:

Teorema 1 Seja $\boldsymbol{X}(t), t=0, \pm 1, \pm 2, \ldots$, vetor $r$-dimensional satisfazendo condições de estacionariedade e sendo $B_{T} \rightarrow 0, B_{T} T \stackrel{T \rightarrow \infty}{\rightarrow} \infty$, se $\widehat{\kappa_{X Y}(\omega)}$ é dado como em (2.21) então

$$
\mathbb{E}\left(\widehat{\kappa_{X Y}(\omega)}\right)=\kappa_{X Y}(\omega)+\mathscr{O}\left(B_{T}\right)+\mathscr{O}\left(B_{T}^{-1} T^{-1}\right)
$$

Então, temos que:

$$
\mathbb{E}\left(\widehat{\kappa_{X Y}(\omega)}\right) \approx \kappa_{X Y}(\omega)
$$


portanto é aproximadamente não viesado. Pode-se ainda provar que

$$
\operatorname{Var}\left(\widehat{\kappa_{X Y}(\omega)}\right) \approx \frac{g^{2}}{2}\left\{1-\kappa_{X Y}^{2}(\omega)\right\}
$$

onde:

- $g^{2}=\frac{n^{\prime}}{n} \frac{U_{4}}{U_{2}^{2}} \sum g_{u}^{2}$

- $\frac{U_{4}}{U_{2}^{2}} \approx \frac{128-93 p}{2(8-5 p)^{2}}$

- $p=\frac{n^{\prime}}{n} \mathrm{e}$

- $\left(g_{u}\right)$ denota os pesos utilizados para a suavização.

$\operatorname{Bloomfield}(2000)$ mostra ainda que $\operatorname{cov}\left(\widehat{\phi_{X Y}(\omega)}, \widehat{\kappa_{X Y}(\omega)}\right) \approx 0$. No entanto, vemos que a variância de $\widehat{\kappa_{X Y}(\omega)}$ depende de $\kappa_{X Y}(\omega)$. Uma alternativa é, neste caso, trabalhar com a transformação $\operatorname{arctanh}($.$) . Com isso, teremos:$

$$
\begin{gathered}
\left.E\left(\operatorname{arctanh}\left(\widehat{\kappa_{X Y}(\omega}\right)\right)\right) \approx \operatorname{arctanh}\left(\kappa_{X Y}(\omega)\right), \\
\operatorname{Var}\left(\operatorname{arctanh}\left(\widehat{\kappa_{X Y}(\omega)}\right)\right) \approx \frac{g^{2}}{2} .
\end{gathered}
$$

Portanto, supondo normalidade assintótica de $\operatorname{arctanh}\left(\widehat{\kappa_{X Y}(\omega)}\right)$, então temos que um possível intervalo de confiança para $\widehat{\kappa_{X Y}(\omega)}$ pode ser construído a partir de:

$$
\begin{aligned}
I C[\kappa ; 0,95] & \equiv \operatorname{arctanh}\left(\widehat{\kappa_{X Y}(\omega)}\right) \pm 1,96 \sqrt{\frac{g^{2}}{2}} \\
& =\frac{1}{2} \ln \left[\frac{1+\widehat{\kappa_{X Y}(\omega)}}{1-\widehat{\kappa_{X Y}(\omega)}} \pm 1,96 \sqrt{\frac{g^{2}}{2}}\right]
\end{aligned}
$$

\subsection{Considerações acerca da Coerência via Análie de Fourier}

- Quando a fase é indefinida, o espectro cruzado não existe. De maneira semelhante, para valores muito pequenos na fase, o espectro cruzado é pequeno.

- O viés associado a $\widehat{\kappa_{X Y}(\omega)}$ é de $\frac{g^{2}}{4} \cdot \frac{\left(1-\kappa_{X Y}(\omega)\right)^{2}}{\kappa_{X Y}(\omega)}$, que é sempre positivo.

- Estimativas da fase podem se mostrar imprecisas para valores pequenos de coerência.

- A coerência estimada pode não ter valores entre 0 e 1 , e pode ter viés próximo a $1 / 2$ nos casos que a quantidade de observações das séries é pequena. 


\section{Capítulo 3}

\section{Coerência Parcial}

No capítulo anterior, definimos a função de coerência e discutimos suas principais propriedades. Nete capítulo, introduziremos o conceito de coerência parcial, calculada como a relação entre duas séries após a remoção do efeito linear entre elas. Para tanto, apresentaremos uma notação matricial e a partir daí, construíremos a coerência parcial através de uma filtragem.

\subsection{Filtro de Regressão}

Seja um vetor de dimensão $(r+s), r, s \in \mathbb{Z}$, contendo duas séries multivariadas $\mathbf{X}(t)$ e $\mathbf{Y}(t)$, $t \in \mathbb{Z}$, de dimensões $r$ e $s$, respectivamente,

$$
\left[\begin{array}{l}
\mathbf{X}(t) \\
\mathbf{Y}(t)
\end{array}\right]
$$

Suponha que cada série de (3.1) satisfaz as condições usuais de estacionariedade e assuma

$$
\begin{gathered}
\mathbb{E}[\mathbf{X}(t)]=\boldsymbol{\mu}_{X}, \\
\mathbb{E}[\mathbf{Y}(t)]=\boldsymbol{\mu}_{Y}, \\
\operatorname{Cov}[\mathbf{X}(t), \mathbf{X}(t+u)]=\mathbf{c}_{X X}(u), \\
\operatorname{Cov}[\mathbf{X}(t), \mathbf{Y}(t+u)]=\mathbf{c}_{X Y}(u), \\
\operatorname{Cov}[\mathbf{Y}(t), \mathbf{Y}(t+u)]=\mathbf{c}_{Y Y}(u) .
\end{gathered}
$$

Conseqüentemente, os espectros são definidos por

$$
\begin{aligned}
\mathbf{f}_{X X}(\omega) & =(2 \pi)^{-1} \sum_{u} \mathbf{c}_{X X} e^{-i u \omega} \\
\mathbf{f}_{X Y}(\omega) & =(2 \pi)^{-1} \sum_{u} \mathbf{c}_{X Y} e^{-i u \omega}, \\
\mathbf{f}_{Y Y}(\omega) & =(2 \pi)^{-1} \sum_{u} \mathbf{c}_{Y Y} e^{-i u \omega} .
\end{aligned}
$$

Buscamos um filtro $s \times r$ dimensional a e um vetor $s$ dimensional $\mathbf{b}$ tal que a quantidade

$$
\mathbf{b}+\sum_{u} \mathbf{a}(t-u) \mathbf{X}(u)
$$


seja um valor próximo de $\mathbf{Y}(t)$.

Considere agora a seguinte função

$$
\mathbb{E}\left[(\mathbf{Y}-\mathbf{b}-\mathbf{a X})(\mathbf{Y}-\mathbf{b}-\mathbf{a X})^{\prime}\right]
$$

Brillinger(2001) sugere a seguinte escolha como minimização para a expressão (3.6) acima:

$$
\begin{aligned}
\mathbf{b} & =\boldsymbol{\mu}_{Y}-\mathbf{c}_{Y X} \mathbf{c}_{X X}^{-1} \boldsymbol{\mu}_{X}, \\
\mathbf{a} & =\mathbf{c}_{Y X} \mathbf{c}_{X X}^{-1}
\end{aligned}
$$

com os quais a expressão (3.6) vale

$$
\mathbf{c}_{Y Y}-\mathbf{c}_{Y X} \mathbf{c}_{X X}^{-1} \mathbf{c}_{X Y}
$$

O filtro a é chamado de coeficiente de regressão de $\boldsymbol{Y}$ em $\boldsymbol{X}$.

A expressão

$$
\boldsymbol{\mu}_{Y}+\mathbf{c}_{Y X} \mathbf{c}_{X X}^{-1}\left(\mathbf{X}-\boldsymbol{\mu}_{X}\right)
$$

é chamada de melhor preditor linear de $\boldsymbol{Y}$ baseado em $\boldsymbol{X}$. Podemos, a partir daí, definir o erro cometido pela estimação como

$$
\boldsymbol{\epsilon}=\mathbf{Y}-\boldsymbol{\mu}_{Y}-\mathbf{c}_{Y X} \mathbf{c}_{X X}^{-1}\left(\mathbf{X}-\boldsymbol{\mu}_{X}\right)
$$

Esta expressão representa o resíduo gerado pela aproximação de $\mathbf{Y}$ pela melhor função linear de $\mathbf{X}$. A matriz de covariância de $\epsilon$ é dada por

$$
\mathbf{c}_{\epsilon \epsilon}=\mathbf{c}_{Y Y}-\mathbf{c}_{Y X} \mathbf{c}_{X X}^{-1} \mathbf{c}_{X Y}
$$

Note que este valor é equivalente à expressão (3.8), para a qual (3.6) tem valor mínimo. Assim, dadas duas componentes de $\mathbf{Y}, Y_{j}$ e $Y_{k}$, a covariância entre $\epsilon_{j}$ e $\epsilon_{k}$ é chamada de covariância parcial de $Y_{j}$ e $Y_{k}$. Pode-se interpretá-la como a relação linear entre $Y_{j}$ e $Y_{k}$ após a remoção do efeito de $\mathbf{X}$. Analogamente, a correlação entre $\epsilon_{j}$ e $\epsilon_{k}$ é denominada de correlação parcial de $Y_{j}$ e $Y_{k}$ após a remoção do efeito de $\mathbf{X}$.

Voltemos agora para a equação (3.5). Buscamos $\mathbf{a}_{s \times r}$ e $\mathbf{b}_{s \times 1}$ tais que (3.5) seja uma boa aproximação para $\mathbf{Y}(t)$. Considere, para tanto a seguinte matriz

$$
\mathbb{E}\left\{\left[\mathbf{Y}(t)-\mathbf{b}-\sum_{u} \mathbf{a}(t-u) \mathbf{X}(u)\right]\left[\mathbf{Y}(t)-\mathbf{b}-\sum_{u} \mathbf{a}(t-u) \mathbf{X}(u)\right]^{\prime}\right\}
$$


Então temos o resultado seguinte ${ }^{1}$ :

Teorema 2 Considere uma série temporal multivariada de dimensão $(r+s)$ fracamente estacionária, como descrita em (3.1), com médias e funções de covariâncias respectivamente como em (3.2) $e$ (3.3). Suponha $\mathbf{c}_{X X}(u)$ e $\mathbf{c}_{Y Y}(u)$ absolutamente somáveis e suponha que $\mathbf{f}_{X X}(\omega)$ dado em (3.4) é não singular, com $-\infty<\omega<\infty$. Então os valores de $\boldsymbol{b}$ e $\boldsymbol{a}(t)$ que minimizam (3.12) são dados por

$$
\boldsymbol{b}=\boldsymbol{\mu}_{y}-\left(\sum_{u} \boldsymbol{a}(u)\right) \boldsymbol{\mu}_{x}=\boldsymbol{\mu}_{y}-\boldsymbol{A}(0) \boldsymbol{\mu}_{x}
$$

$e$

$$
\boldsymbol{a}(u)=(2 \pi)^{-1} \int_{0}^{2 \pi} \boldsymbol{A}(\alpha) e^{i u \alpha} d \alpha
$$

com

$$
\boldsymbol{A}(\omega)=\mathbf{f}_{Y X}(\omega) \mathbf{f}_{X X}(\omega)^{-1}
$$

O filtro $\{\boldsymbol{a}(u)\}$ é absolutamente somável e o minimo alcançado é

$$
\int_{0}^{2 \pi}\left[\mathbf{f}_{Y Y}(\alpha)-\mathbf{f}_{Y X}(\alpha) \mathbf{f}_{X X}(\alpha)^{-1} \mathbf{f}_{X Y}(\alpha)\right] d \alpha .
$$

Conseqüentemente, temos que a série de erros resultante da aproximação de $\mathbf{Y}(t)$ por $\mathbf{X}(t)$ é dada por

$$
\boldsymbol{\epsilon}(t)=\mathbf{Y}(t)-\mathbf{b} \sum_{u} \mathbf{a}(t-u) \mathbf{X}(u)
$$

$t=0, \pm 1, \pm 2, \ldots$ A série $\boldsymbol{\epsilon}(\mathrm{t})$ tem média zero e matriz de densidade espectral dada por

$$
\mathbf{f}_{\epsilon \epsilon}(\omega)=\mathbf{f}_{Y Y}(\omega) \cdot-\mathbf{f}_{Y X}(\omega) \mathbf{f}_{X X}(\omega)^{-1} \mathbf{f}_{X Y}(\omega)
$$

A expressão (3.19) é chamada de espectro residual e pode ser reescrita como

$$
\mathbf{f}_{\epsilon \epsilon}(\omega)=\mathbf{f}_{Y Y}(\omega)^{\frac{1}{2}}\left[\mathbf{I}-\mathbf{f}_{Y Y}(\omega)^{-\frac{1}{2}} \mathbf{f}_{Y X}(\omega) \mathbf{f}_{X X}(\omega)^{-1} \mathbf{f}_{X Y}(\omega) \mathbf{f}_{Y Y}(\omega)^{-\frac{1}{2}}\right] \mathbf{f}_{Y Y}(\omega)^{\frac{1}{2}}
$$

e portanto podemos considerar como medida de associação entre $\mathbf{Y}(t)$ e $\mathbf{X}(t)$ a seguinte expressão,

$$
\mathbf{f}_{Y Y}(\omega)^{-\frac{1}{2}} \mathbf{f}_{Y X}(\omega) \mathbf{f}_{X X}(\omega)^{-1} \mathbf{f}_{X Y}(\omega) \mathbf{f}_{Y Y}(\omega)^{-\frac{1}{2}}
$$

\footnotetext{
${ }^{1}$ Veja Brillinger(2001).
} 
No caso em que $\mathrm{s}=1,(3.21)$ é chamada de coerência múltipla e é definida por

$$
\left|K_{X Y}^{2}(\omega)\right|=\frac{\mathbf{f}_{Y X}(\omega) \mathbf{f}_{X X}^{-1}(\omega) \mathbf{f}_{X Y}(\omega)}{f_{Y Y}(\omega)}
$$

e é uma generalização do caso $\mathrm{r}=\mathrm{s}=1$, apresentado em (2.17).

\subsection{Função de Coerência Parcial}

Dessa maneira, considerando o a-ésimo e o b-ésimo componentes de $\boldsymbol{\epsilon}(\mathrm{t}), \epsilon_{a}(\mathrm{t})$ e $\epsilon_{b}(\mathrm{t})$, seu espectro cruzado pode ser interpretado como o espectro parcial cruzado de $Y_{a}(\mathrm{t})$ e $Y_{b}(\mathrm{t})$, a-ésimo e o b-ésimo componentes de $\mathbf{Y}(t)$, após removidos os efeitos de $\mathbf{X}(t)$. Considerando a discussão da seção anterior, podemos calcular o Espectro Cruzado Parcial da seguinte maneira:

$$
f_{Y_{a} Y_{b}, X}(\omega)=f_{Y_{a}, Y_{b}}(\omega)-\mathbf{f}_{Y_{a}, X}(\omega) \mathbf{f}_{X X}(\omega)^{-1} \mathbf{f}_{X, Y_{b}}(\omega)=f_{\epsilon_{a}, \epsilon_{b}}(\omega) .
$$

Note que a definição é a mesma apresentada em (3.19). A coerência entre esses elementos $\epsilon_{a}(\mathrm{t})$ e $\epsilon_{b}(\mathrm{t})$ é chamada de Coerência Parcial entre $Y_{a}(t)$ e $Y_{b}(t)$, após removido o efeito de $\mathbf{X}(t)$ e é portanto dada por

$$
\mathbf{R}_{Y_{a} Y_{b}, X}(\omega)=\frac{f_{Y_{a} Y_{b}, X}(\omega)}{\left[f_{Y_{a} Y_{a}, X}(\omega) f_{Y_{b} Y_{b}, X}(\omega)\right]^{1 / 2}}
$$

\subsection{Estimação de $\mathbf{R}_{Y_{a} Y_{b}, X}$}

Considere que temos observados o vetor $(\mathrm{r}+\mathrm{s})$-dimensional dado em (3.1) para $\mathrm{t}=0, \ldots, \mathrm{T}-1$. Calculando sua transformada discreta de Fourier,temos que

$$
\left[\begin{array}{c}
\mathbf{d}_{X}^{(T)}(\omega) \\
\mathbf{d}_{Y}^{(T)}(\omega)
\end{array}\right]=\sum_{t=0}^{T-1} e^{-i \omega t}\left[\begin{array}{c}
\mathbf{X}(t) \\
\mathbf{Y}(t)
\end{array}\right]
$$

$\omega \in \mathbb{R}$.

Sabemos $^{2}$ que para $\mathrm{T}$ suficientemente grande e $\omega \neq 0,(3.25)$ tem distribuição assintótica dada por

$$
\left[\begin{array}{l}
\mathbf{d}_{X}^{(T)}(\omega) \\
\mathbf{d}_{Y}^{(T)}(\omega)
\end{array}\right] \sim N_{r+s}^{c}\left(0,2 \pi T\left[\begin{array}{cc}
\mathbf{f}_{X X}(\omega) & \mathbf{f}_{X Y}(\omega) \\
\mathbf{f}_{Y X}(\omega) & \mathbf{f}_{Y Y}(\omega)
\end{array}\right]\right)
$$

Com isso, definimos a matriz de periodograma cruzados

$$
\mathbf{I}_{X Y}^{T}(\omega)=(2 \pi T)^{-1} \mathbf{d}_{X}^{(T)}(\omega)\left(\overline{\mathbf{d}_{Y}^{(T)}(\omega)}\right)^{\tau},
$$

com definições análogas para $\mathbf{I}_{X X}^{T}(\omega)$ e $\mathbf{I}_{Y Y}^{T}(\omega)$.

\footnotetext{
${ }^{2}$ Veja Brillinger (2001)
} 
Considere agora uma família de pesos $2 \pi$-periódica, $W^{(T)}(\alpha),-\infty<\alpha<\infty, \mathrm{T}=1,2, \ldots$, cujos pesos estão arranjados de maneira adequada para estimar (3.4). Considere ainda uma seqüência de parâmetros de escala $B_{T}>0, B_{T} \rightarrow 0, B_{T} T^{T \rightarrow \infty} \rightarrow \infty$ e uma função $W(\alpha)$ que goza das seguintes propriedades:

$$
\begin{gathered}
\int_{-\infty}^{\infty} W(\alpha) d \alpha=1, \\
\int_{-\infty}^{\infty}|W(\alpha)| d \alpha<\infty .
\end{gathered}
$$

Pode-se construir ${ }^{3} W^{(T)}(\alpha)$ a partir de

$$
W^{(T)}(\alpha)=\sum_{j=-\infty}^{\infty} B_{T}^{-1} W\left(B_{T}^{-1}[\alpha+2 \pi j]\right),-\infty<\alpha<\infty
$$

Finalmente, estimamos a matriz

$$
\left[\begin{array}{ll}
\mathbf{f}_{X X}(\omega) & \mathbf{f}_{X Y}(\omega) \\
\mathbf{f}_{Y X}(\omega) & \mathbf{f}_{Y Y}(\omega)
\end{array}\right]
$$

por

$$
\left[\begin{array}{cc}
\mathbf{f}_{X X}^{(T)}(\omega) & \mathbf{f}_{X Y}^{(T)}(\omega) \\
\mathbf{f}_{Y X}^{(T)}(\omega) & \mathbf{f}_{Y Y}^{(T)}(\omega)
\end{array}\right]=(2 \pi T)^{-1} \sum_{s=0}^{T-1} W^{(T)}\left(\omega-\frac{2 \pi s}{T}\right)\left[\begin{array}{cc}
\mathbf{I}_{X X}^{(T)}\left(\frac{2 \pi s}{T}\right) & \mathbf{I}_{X Y}^{(T)}\left(\frac{2 \pi s}{T}\right) \\
\mathbf{I}_{Y X}^{(T)}\left(\frac{2 \pi s}{T}\right) & \mathbf{I}_{Y Y}^{(T)}\left(\frac{2 \pi s}{T}\right)
\end{array}\right]
$$

A partir dessas estimativas, podemos então estimar a matriz de densidade espectral residual por

$$
\mathbf{f}_{\epsilon \epsilon}^{(T)}(\omega)=\mathbf{f}_{Y Y}^{(T)}(\omega)-\mathbf{f}_{Y X}^{(T)}(\omega) \mathbf{f}_{X X}^{(T)}(\omega)^{-1} \mathbf{f}_{X Y}^{(T)}(\omega) .
$$

O estimador da coerência parcial considerando (3.29), é dado por

$$
\mathbf{R}_{Y_{a} Y_{b}, X}^{(T)}(\omega)=\frac{f_{\epsilon_{a} \epsilon_{b}}^{(T)}(\omega)}{\left[f_{\epsilon_{a} \epsilon_{a}}^{(T)}(\omega) f_{\epsilon_{b} \epsilon_{b}}^{(T)}(\omega)\right]^{1 / 2}}
$$

\subsection{Distribuição assintótica de $\mathbf{R}_{Y_{a} Y_{b}, X}^{(T)}$}

Considerando a matriz (3.1) com as suposições feitas anteriormente e assumindo que $\mathbf{f}_{X X}\left(\omega^{(l)}\right), l=$ $1, \ldots, L$, não-singular, então temos que $\mathbf{R}_{Y_{a} Y_{b}, X}^{(T)}(\omega)$ tem distribuição assintótica normal multivariada com estrutura de covariância dada por

\footnotetext{
${ }^{3}$ Veja Brillinger (2001), p.147
} 


$$
\begin{aligned}
\operatorname{cov}\left(\left|\mathbf{R}_{Y_{a} Y_{b}, X}^{(T)}(\omega)\right|^{2},\left|\mathbf{R}_{Y_{a} Y_{b}, X}^{(T)}(\lambda)\right|^{2}\right) & \\
& {[\nu\{\omega-\lambda\}+\nu\{\omega+\lambda\}]\left|\mathbf{R}_{Y_{a} Y_{b}, X}^{(T)}(\omega)\right|^{2} \times } \\
& {\left[1-\left.\mathbf{R}_{Y_{a} Y_{b}, X}^{(T)}(\omega)\right|^{2}\right]^{2} 4 \pi \int W(\alpha)^{2} d \alpha B_{T^{-1}} T^{-1}+O\left(B_{T^{-2}} T^{-2}\right) . }
\end{aligned}
$$

Para um dado J, as variáveis $\mathbf{R}_{Y_{a} Y_{b}, X}^{(T)}\left(\omega_{1}\right), \ldots, \mathbf{R}_{Y_{a} Y_{b}, X}^{(T)}\left(\omega_{J}\right)$ são assintóticamente conjuntamente normais com estrutura de covariância dada em (3.32) para $1 \leq a<b \leq r$. 


\section{Capítulo 4}

\section{Coerência Parcial Direcionada}

A partir de estudos de Saito e Harashima (1981) sobre a Coerência Direcionada (DC), Baccalá e Sameshima(2001) definem Coerência Parcial Direcionada (PDC). Veremos, a partir de resultados de Takahashi et al. (2007) que há uma relação direta entre a existência de causalidade de Granger e a PDC. Para tanto, definiremos brevemente causalidade de Granger mas não nos atentaremos a discutir tal conceito ${ }^{1}$. Em linhas gerais, a Coerência Parcial Direcionada é a decomposição da coerência parcial a partir de modelos autoregressivos multivariados. Esse conceito pode ser interpretado como uma representação do conceito de causalidade de Granger no domínio da freqüência.

\subsection{Coerência Direcionada - Primeiros Conceitos}

Há muitas limitações quando tratamos de interpretação das funções de coerência descritas até agora. Segundo Baccalá e Sameshima(2001), podemos com as funções de coerência e coerência parcial, ter indícios das relações entre as séries temporais em estudo. Por conta disso, Saito e Harashima (1981) propõem como medida de relação entre séries temporais, a função de coerência direcionada. Esta função nos dá não apenas a idéia de sincronia entre as séries de tempo, mas também indícios de uma conexão funcional entre elas. Neste contexto, eles descrevem a idéia de coerência direcionada como uma função que não apenas nos conta sobre a sincronia das séries em estudo mas também a conexão funcional entre as séries. É possível, portanto, dar importância a relações estruturais relativas, por meio de decomposições da interação das séries em aspectos feedback e feedfoward de maneira unívoca.

Considere assim a matriz de espectros e espectros cruzados

$$
\mathbf{f}(\omega)=\left[\begin{array}{cccc}
f_{11}(\omega) & f_{12}(\omega) & \ldots & f_{1 N}(\omega) \\
f_{21}(\omega) & f_{22}(\omega) & \ldots & f_{2 N}(\omega) \\
\vdots & \vdots & \ddots & \vdots \\
f_{N 1}(\omega) & f_{N 2}(\omega) & \ldots & f_{N N}(\omega)
\end{array}\right]
$$

onde $f_{i j}(\omega), i, j=1, \ldots, N$ são definidos como em (2.10). Temos então que a matriz (4.1) pode ser

\footnotetext{
${ }^{1}$ Mais detalhes podem ser vistos em Morettin (2008).
} 
decomposta na forma

$$
\mathbf{f}(\omega)=\mathbf{H}(\omega) \boldsymbol{\Sigma} \mathbf{H}^{H}(\omega)
$$

onde $\mathbf{H}^{H}$ (.) representa a matriz hermitiana transposta de $\mathbf{H}($.$) e \boldsymbol{\Sigma}$ é a matriz de covariância $\left\{\sigma_{i j}, i, j=1, \ldots, N\right\}$. Para definirmos a matriz $\mathbf{H}$, considere $X_{1}(t), \ldots, X_{N}(t)$ séries temporais conjuntamente estacionárias de tal maneira que tenhamos uma aproximação por meio de modelos autoregressivos

$$
\left[\begin{array}{c}
X_{1}(t) \\
\vdots \\
X_{N}(t)
\end{array}\right]=\sum_{r=1}^{p} \mathbf{A}_{r}\left[\begin{array}{c}
X_{1}(t-r) \\
\vdots \\
X_{N}(t-r)
\end{array}\right]+\left[\begin{array}{c}
w_{1}(t) \\
\vdots \\
w_{N}(t)
\end{array}\right]
$$

na qual $w_{1}(t), \ldots, w_{N}(t)$ sejam ruídos brancos.

A matriz $\mathbf{H}(\omega)$ é dada por

$$
\mathbf{H}(\omega)=\overline{\mathbf{A}}^{-1}(\omega)=(\mathbf{I}-\mathbf{A}(\omega))^{-1}
$$

$$
\begin{gathered}
\mathbf{A}(\omega)=\left.\sum_{r=1}^{p} \mathbf{A}_{r} z^{-r}\right|_{z=e^{-i 2 \pi \omega}}= \\
=\sum_{r=1}^{p} \mathbf{A}_{r} e^{i 2 \pi \omega r} \\
\mathbf{A}_{r}=\left[\begin{array}{cccc}
a_{11}(r) & a_{12}(r) & \ldots & a_{1 N}(r) \\
\vdots & \vdots & \ddots & \vdots \\
a_{N 1}(r) & a_{N 2}(r) & \ldots & a_{N N}(r)
\end{array}\right]
\end{gathered}
$$

onde $a_{i j}(r)$ são os coeficientes que representam o efeito de interação linear de $x_{j}(t-r)$ com $x_{i}(t)$, $i, j=1, \ldots, N$.

\subsection{Causalidade de Granger}

Sejam $\mathrm{X}(\mathrm{t})$ e $\mathrm{Y}(\mathrm{t})$ duas séries temporais. Dizemos que $\mathrm{X}(\mathrm{t})$ Granger-causa $\mathrm{Y}(\mathrm{t})$ se a informação contida no passado de $\mathrm{X}(\mathrm{t})$ aumenta nossa precisão em relação a previsões sobre o presente e futuro de $\mathrm{Y}(\mathrm{t})$. Essa relação entre as séries $\mathrm{X}(\mathrm{t})$ e $\mathrm{Y}(\mathrm{t})$ é unidirecional, o que sugere uma relação de conectividade entre as duas séries em questão. Para nós, duas delas são particularmente interessantes:

Seja $\mathscr{U}(t)$ toda a informação acumulada no universo de estudo até o instante t. Dessa maneira, $\mathscr{U}(t)-Y(t)$ denota toda a essa informação exceto aquela contida em $Y(t)$. Considere ainda $X(t)$ 
e $Y(t)$ duas séries temporais contidas em $\mathscr{U}(t)$. Defina $\overline{X_{t}}:=\left\{X_{s}: s<t\right\}, \overline{\overline{X_{t}}}=\left\{X_{s}: s \leq t\right\}$. Analogamente, defina $\overline{\overline{Y_{t}}}$ e $\overline{Y_{t}}$. Seja ainda $P_{t}(Y \mid \mathscr{U})$ o preditor do EQM mínimo de $Y_{t}$, dada a informação contida em $\mathscr{U}$ e $\sigma^{2}(Y \mid \mathscr{U})$ seu correspondente EQM.

Definição 3 (Causalidade) Se $\sigma^{2}(Y(t) \mid \overline{\mathscr{U}(t)})<\sigma^{2}\left(Y(t) \mid \overline{\mathscr{U}_{t}}-\overline{X_{t}}\right)$ então dizemos que $X(t)$ "Grangercausa" $^{\prime \prime} Y(t)$.

Definição 4 (Causalidade Instantânea) Considere $\mathscr{U}(t), Y(t), X(T)$ e $Y(t)$ como na definição 3. Se $\sigma^{2}\left(Y(t) \mid \overline{U_{t}}, \overline{\overline{X_{t}}}\right)<\sigma\left(Y(t) \mid \overline{\left.U_{t}\right)}\right.$ então dizemos que há relação de causalidade instantânea de $X(t)$ para $Y(t)$. Em outras palavras, o presente de $Y(t)$ é melhor predito se o presente de $X(t) e ́$ incluído no modelo de predição.

É usual na literatura ${ }^{2}$ apresentar testes para a existência da causalidade de Granger relacionados com os coeficientes apresentados em um modelo linear. Considere, portanto, a equação (4.3). Testar a causalidade de Granger é equivalente a testar:

$$
H: a_{i j}(r)=0, \forall r \in\{1, \ldots, p\}
$$

Segundo Sato et. al. (2009), embora conceitualmente interessante, a causalidade de Granger tem sua forma original definida no dominio do tempo, o que não nos permite discernir a cerca de características dos sinais no dominio da freqüência. E ainda que não é clara a maneira de definir a causalidade de Granger no contexto multivariado para se obter uma normalização poderosa e adequada para comparações entre os valores.

\subsection{Coerência Parcial Direcionada}

Considere a função de coerência parcial definida em (3.24). Podemos reescrevê-la em função de $A_{r}$ e $\boldsymbol{\Sigma}$ como se segue:

$$
\mathbf{R}_{Y_{i} Y_{j}, X}(\omega)=\frac{\overline{\mathbf{a}}_{i}^{H}(\omega) \boldsymbol{\Sigma}^{-1} \overline{\mathbf{a}}_{j}(\omega)}{\sqrt{\left(\overline{\mathbf{a}}_{i}^{H}(\omega) \boldsymbol{\Sigma}^{-1} \overline{\mathbf{a}}_{i}(\omega)\right)\left(\overline{\mathbf{a}}_{j}^{H}(\omega) \boldsymbol{\Sigma}^{-1} \overline{\mathbf{a}}_{j}(\omega)\right)}}
$$

onde $\boldsymbol{\Sigma}$ é a matriz de covariância do erro cometido na predição associada ao modelo dado em (4.3) e $\overline{\mathbf{a}}_{k}(\omega)$ representa a k-ésima coluna da matriz $\overline{\mathbf{A}}(\omega)$.

Definição $5 O$ Fator de Coerência Parcial Direcionada entre duas séries temporais $X_{i}(t)$ e $X_{j}(t)$ é dado por

$$
\pi_{i j}(\omega):=\frac{\bar{A}_{i j}(\omega)}{\sqrt{\overline{\boldsymbol{a}}_{j}^{H}(\omega) \boldsymbol{\Sigma}^{-1} \overline{\boldsymbol{a}}_{j}(\omega)}},
$$

onde $\bar{A}_{i j}(\omega)$ é o i,j-ésimo elemento de $\overline{\boldsymbol{A}}(\omega), i, j=1 \ldots, N$.

\footnotetext{
${ }^{2}$ Veja, por exemplo, Takahashi et. al. (2007)
} 
É imediato a partir desta definição que (4.8) fica

$$
\mathbf{R}_{Y_{i} Y_{j}, X}(\omega)=\pi_{i}^{H}(\omega) \boldsymbol{\Sigma}^{-1} \pi_{j}(\omega)
$$

onde $\pi_{i}(\omega):=\left[\pi_{1 i}(\omega), \ldots, \pi_{N i}(\omega)\right]^{\prime}$.

Como o denominador de (4.9) depende de $\boldsymbol{\Sigma}$, a coerência parcial confunde efeitos de causalidade de Granger e causalidade instantânea de Granger. Para eliminar este efeito instantâneo, define-se a Coerência Parcial Direcionada como

$$
\bar{\pi}_{i j}(\omega):=\frac{\bar{A}_{i j}(\omega)}{\sqrt{\overline{\mathbf{a}}_{j}^{H}(\omega) \overline{\mathbf{a}}_{j}(\omega)}},
$$

a qual considera apenas causalidade "não-instantânea"de Granger, já que relacionamentos entre observações presentes de $X_{i}(t)$ são descritos exclusivamente pelas correlações entre os processos $w_{i}(t)$ de (4.3). Suprimindo $\boldsymbol{\Sigma}$ de (4.10), focamos nosso objeto de estudo na relação entre os valores passados das séries $X_{j}(t)$ e o presente e futuro das séries $X_{i}(t)$.

Note que $\bar{A}_{i j}(\omega)$ pode ser escrito como:

$$
\bar{A}_{i j}(\omega)=\left\{\begin{array}{lc}
1-\sum_{r=1}^{p} a_{i j}(r) e^{-i 2 \pi \omega r} & , \text { se } \mathrm{i}=\mathrm{j} \\
-\sum_{r=1}^{p} a_{i j}(r) e^{-i 2 \pi \omega r} & , \text { caso contrário }
\end{array}\right.
$$

Por conta dessa representação, dizemos que a equação (4.11) é a representação da causalidade de Granger no domínio da freqüência ${ }^{3}$. Segundo Takahashi et. al. (2007), isso se deve ao fato de (4.7) ser verificada se e somente se $\bar{\pi}_{i j}(\omega) \equiv 0$ para todas as frequências de amostragem, $\omega \in[-0.5,0.5]$.

\subsection{Distribuição Assintótica}

Takahashi et. al. (2007) apresentam resultados assintóticos a cerca da coerência parcial direcionada definida em (4.11). Considere a seguinte notação:

$$
\bar{\alpha}(\omega)=\operatorname{vec}(\mathbf{I}) \sum_{r=1}^{p} \operatorname{vec}\left(\mathbf{A}_{r}\right) e^{-i 2 \pi \omega r},
$$

onde I é a matriz identidade de dimensão N, e $\operatorname{vec}\left(\boldsymbol{A}_{r}\right)$ indica a transformação (empilhamento) da matriz $\mathbf{A}_{N \times N}$ no vetor $\mathbf{A}_{N^{2} \times 1}$. Analogamente, vec(I) representa a transformação da matriz $\mathbf{I}_{N \times N}$ no vetor $\mathbf{I}_{N^{2} \times 1}$. Considere ainda

$$
a(\omega)=\left[\begin{array}{c}
\operatorname{Re}(\bar{\alpha}(\omega)) \\
\operatorname{Im}(\bar{\alpha}(\omega))
\end{array}\right]
$$

\footnotetext{
${ }^{3}$ Veja Takahashi et. al. (2007)
} 
Com isso, Takahashi et. al. (2007) provam os seguintes resultados:

Lema 1 Para um processo $\operatorname{VAR}(p)$ gaussiano estacionário estável definido pelo modelo (4.3), o estimador de máxima verosimilhança de a é consistente e

$$
\sqrt{n}(\hat{\boldsymbol{a}}(\omega)-\boldsymbol{a}(\omega)) \stackrel{d}{\rightarrow} \mathscr{N}(0, \bar{\Omega}(\omega))
$$

A forma exata da matriz $\bar{\Omega}(\omega)$ é dada em Takahashi et. al. (2007a), salientando somente o fato de ser uma matriz simétrica positiva semi-definida.

Proposição 3 Para um processo gaussiano estacionário estável, o estimador de máxima verossimilhança $\left|\hat{\pi}_{i j}(\omega)\right|^{2}$ é consistente e assintóticamente normal se $\left|\pi_{i j}(\omega)\right|^{2} \neq 0$, i.e.,

$$
\sqrt{n} \gamma^{-1}\left(\hat{\boldsymbol{a}}\left(\left|\hat{\pi}_{i j}(\omega)\right|^{2}-\left|\pi_{i j}(\omega)\right|^{2}\right)\right) \stackrel{d}{\rightarrow} \mathscr{N}(0,1)
$$

onde

$$
\begin{gathered}
\gamma^{2}(\overline{\mathbf{a}})=\mathbf{G}(\hat{\mathbf{a}})^{\prime} \hat{\bar{\Omega}} \mathbf{G}(\hat{\mathbf{a}}), \\
\mathbf{G}(\hat{\mathbf{a}})=2\left(\mathbf{I}_{i j}^{c} \hat{\mathbf{a}}\right)\left(\hat{\mathbf{a}}^{\prime} \mathbf{I}_{j}^{c} \hat{\mathbf{a}}\right)^{-1}-2\left(\mathbf{I}_{j}^{c} \hat{\mathbf{a}}\right)\left(\hat{\mathbf{a}}^{\prime} \mathbf{I}_{j}^{c} \hat{\mathbf{a}}\right)^{-2}\left(\hat{\mathbf{a}}^{\prime} \mathbf{I}_{i j}^{c} \hat{\mathbf{a}}\right), \\
\mathbf{I}_{i j}^{c}=\left[\begin{array}{cc}
\mathbf{I}_{i j} & 0 \\
0 & \mathbf{I}_{i j}
\end{array}\right], \\
\mathbf{I}_{j}^{c}=\left[\begin{array}{cc}
\mathbf{I}_{j} & 0 \\
0 & \mathbf{I}_{j}
\end{array}\right],
\end{gathered}
$$

onde a matriz $\mathbf{I}_{i j}$ de dimensão $N^{2} \times N^{2}$ é composta de zeros, exceto valores das coordenadas $(\mathrm{l}, \mathrm{m})=((\mathrm{j}-1) \mathrm{N}+\mathrm{i},(\mathrm{j}-1) \mathrm{N}+\mathrm{i})$, que são iguais a 1. Analogamente, os blocos $\mathbf{I}_{j}$ tem dimensão $N^{2} \times N^{2}$ com valores zero, exceto nas coordenadas $(1, m):(j-1) N+1 \leq l=m \leq j N$, os quais são iguais a 1 . Detalhes podem ser encontrados em Takahashi et. al. (2007) e Takahashi et. al. (2007a). 


\section{Capítulo 5}

\section{Aplicações}

Neste capítulo, simularemos um VAR(p) trivariado, processo auto-regressivo multivariado e a partir das funções estudadas, aplicaremos os conceitos de coerência, coerência parcial e coerência parcial direcionada para estudar o relacionamento entre as séries que compõem o VAR(p). Utilizaremos também dados de medições de eletroencefalograma (EEG) em estado de vigília.

\subsection{Resultados a partir de simulações}

Utilizando o pacote mAr e sua função mAr.sim (desenvolvido por Barbosa, 2008) simulamos 10.000 observações do seguinte processo:

$$
\left[\begin{array}{l}
X_{1}(t) \\
X_{2}(t) \\
X_{3}(t)
\end{array}\right]=\left[\begin{array}{c}
0.4 \\
-0.17 \\
-0.25
\end{array}\right]+\left[\begin{array}{ccc}
0.25 & 0.3 & 0.2 \\
-0.5 & -0.16 & -0.1 \\
0.3 & 0.1 & 0.15
\end{array}\right]\left[\begin{array}{c}
X_{1}(t-1) \\
X_{2}(t-1) \\
X_{3}(t-1)
\end{array}\right]+\epsilon_{3}(t)
$$

em que

$$
\epsilon_{\mathbf{3}} \sim R B_{3}(\mathbf{0}, \boldsymbol{\Sigma}), \boldsymbol{\Sigma}=\left[\begin{array}{ccc}
1 & 0.2 & 0.1 \\
0.2 & 1 & 0.1 \\
0.1 & 0.1 & 1
\end{array}\right]
$$

Nas figuras 5.1, 5.2 e 5.3 temos respectivamente (a) o gráfico da série, (b) o histograma dos dados, (c) a função de auto-correlação e (d) a função de auto-correlação parcial para as séries $X_{1}(t), X_{2}(t)$ e $X_{3}(t)$ do processo descrito em (5.1).

O gráfico apresentado na figura 5.4 apresenta as coerências entre as séries $X_{1}(t), X_{2}(t)$ e $X_{3}(t)$. Note que há uma maior dependência entre as séries $X_{1}(t)$ e $X_{2}(t)$, principalmente nas frequências abaixo de 0.25. As funções de coerência para $X_{1}(t)$ e $X_{3}(t)$ e $X_{2}(t)$ e $X_{3}(t)$ mostram-se em geral mais baixas que 0.4 , o que mostra uma baixa influência de $X_{3}(t)$.

Temos nas figuras 5.5, 5.6 e 5.7, respectivamente, representando o módulo da coerências parciais entre $X_{1}(t)$ e $X_{2}(t), X_{1}(t)$ e $X_{3}(t)$ e $X_{2}(t)$ e $X_{3}(t)$. As funções utilizadas na estimação da curva foram baseadas em Bacallá et al.(2006) e os programas utilizados encontram-se no apêndice A(sintaxe R).

Na figura 5.8, 5.9 e 5.10 temos o quadrado do módulo das PDCs entre as séries simuladas. Temos respectivamente as relações entre $X_{1}(t)$ e $X_{2}(t), X_{1}(t)$ e $X_{3}(t)$ e $X_{2}(t)$ e $X_{3}(t)$. Para o cálculo da 

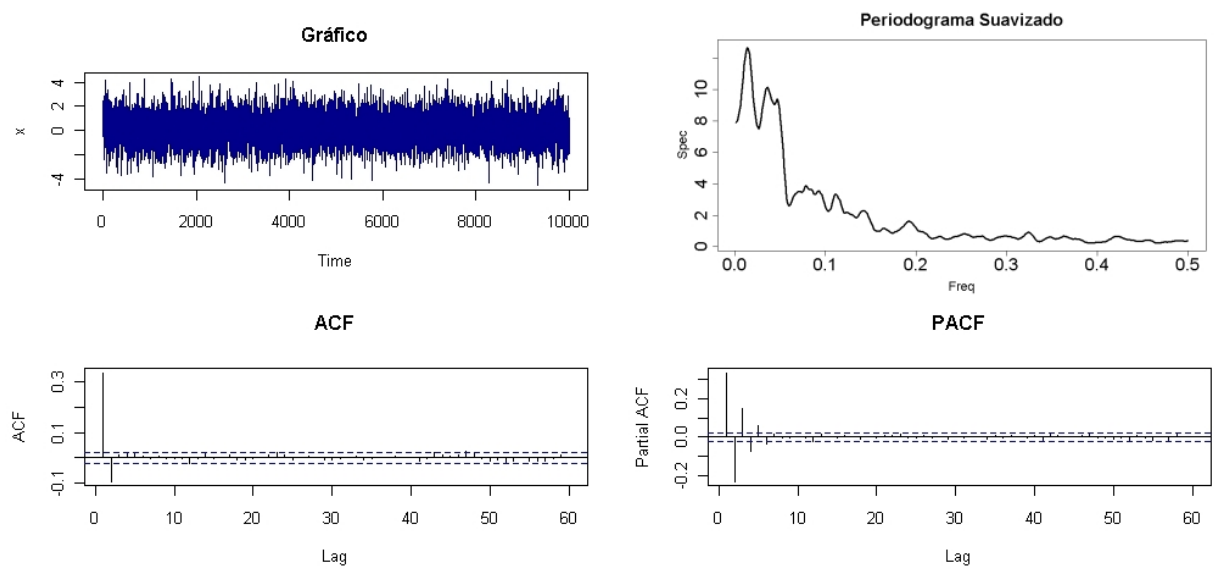

Figura 5.1: Gráficos da série $X_{1}(t)$.
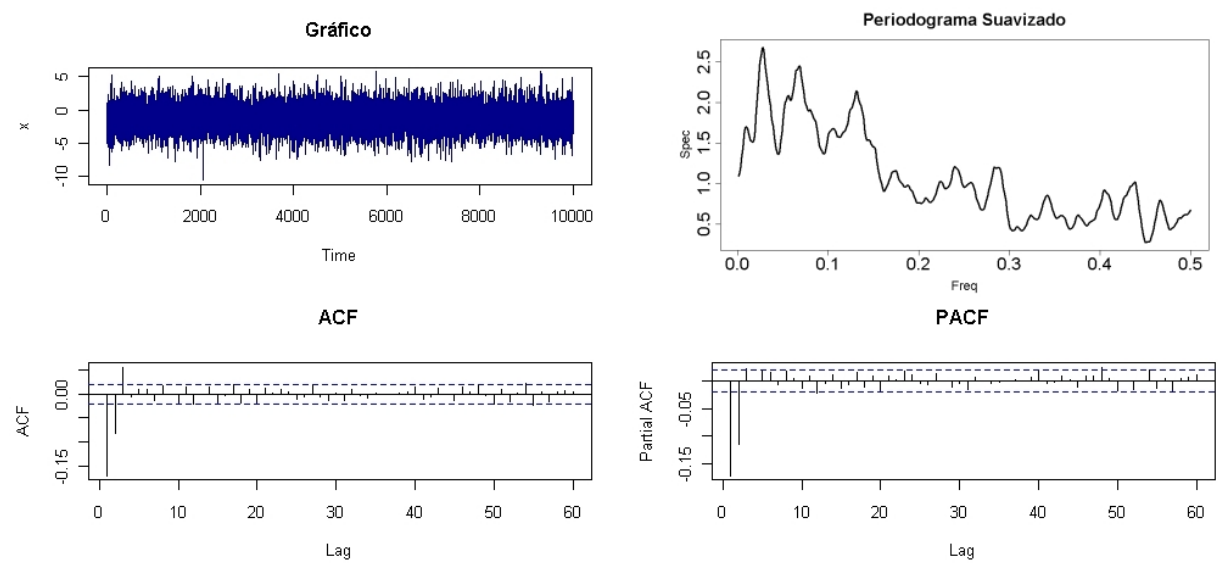

Figura 5.2: Gráficos da série $X_{2}(t)$.
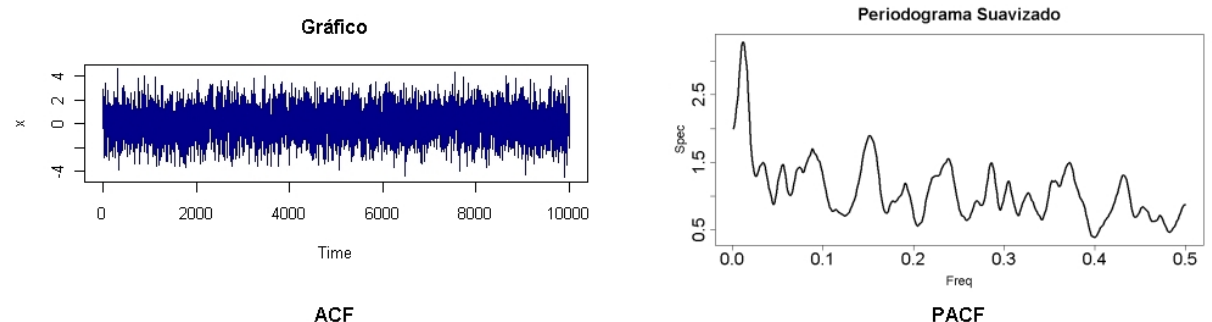

ACF
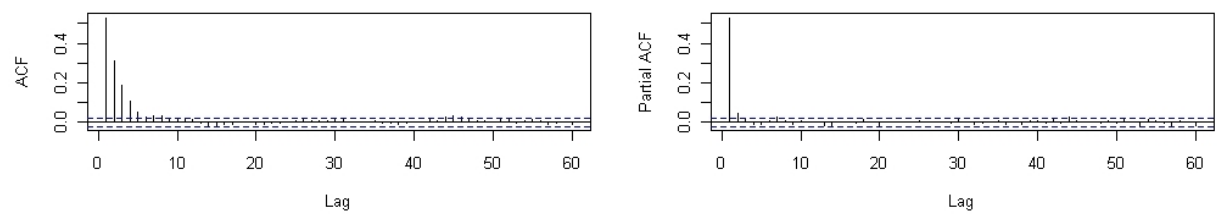

Figura 5.3: Gráficos da série $X_{3}(t)$. 

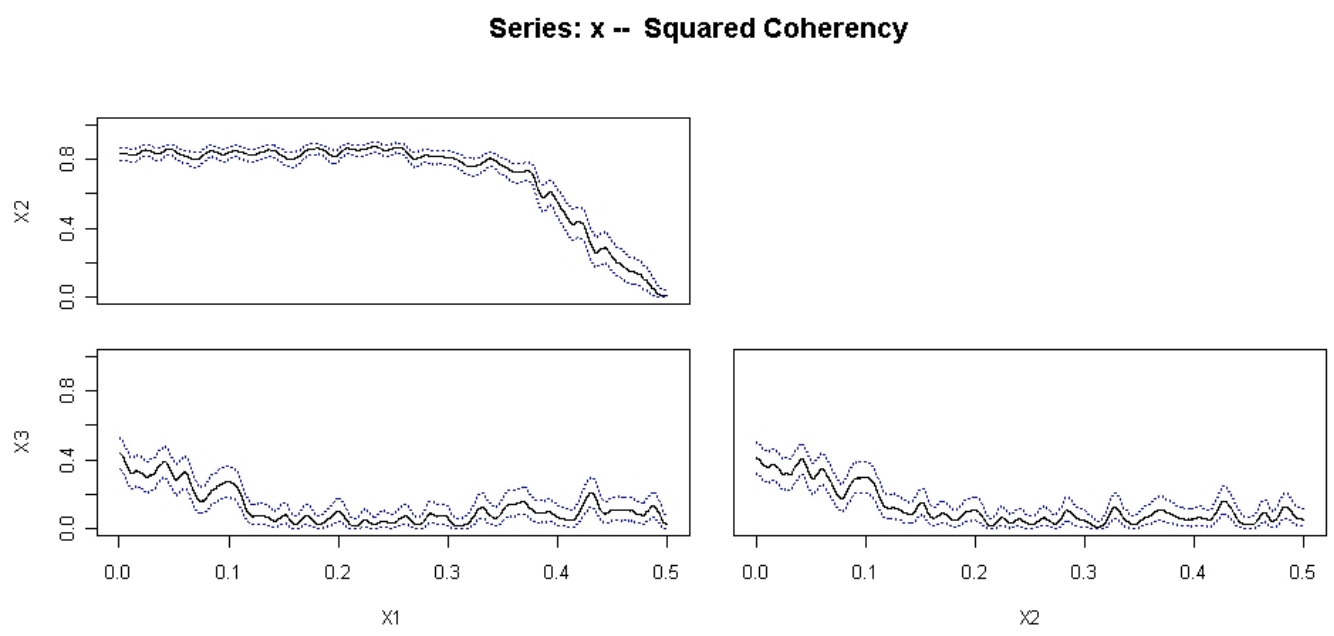

Figura 5.4: Funções de coerência $X_{1}(t), X_{2}(t)$ e $X_{3}(t)$.

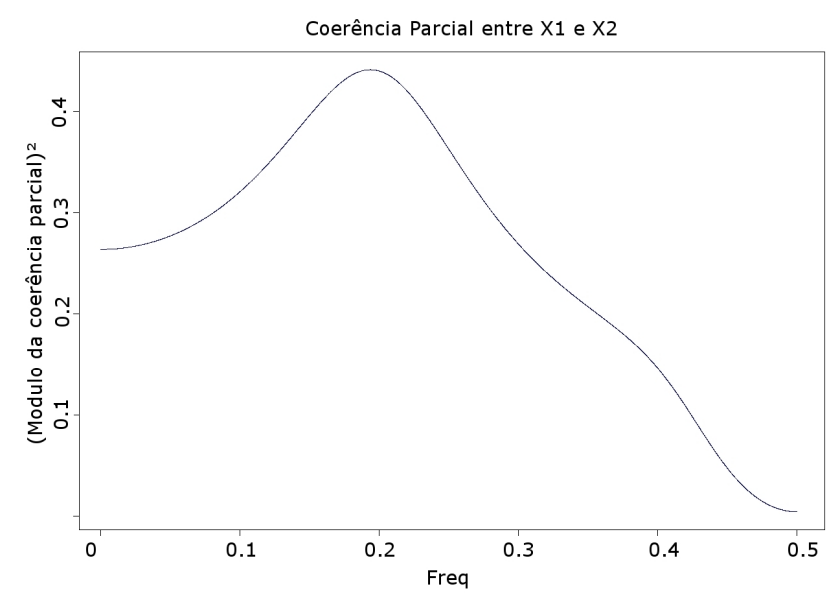

Figura 5.5: Módulo da função de coerência parcial entre $X_{1}(t)$ e $X_{2}(t)$.

funções de coerência parcial utilizamos o critério de Akaike(AIC) para comparação dos modelos, limitado a um VAR(10) para o ajuste dos dados. Já para o cálculo das PDCs utilizamos o critério de AIC limitado a um $\operatorname{VAR}(3)$. 


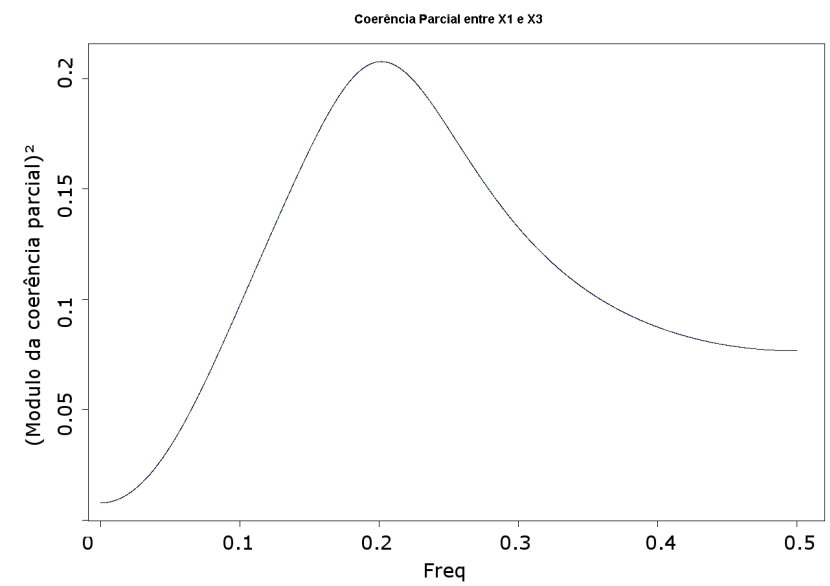

Figura 5.6: Módulo da função de coerência parcial entre $X_{1}(t)$ e $X_{3}(t)$.

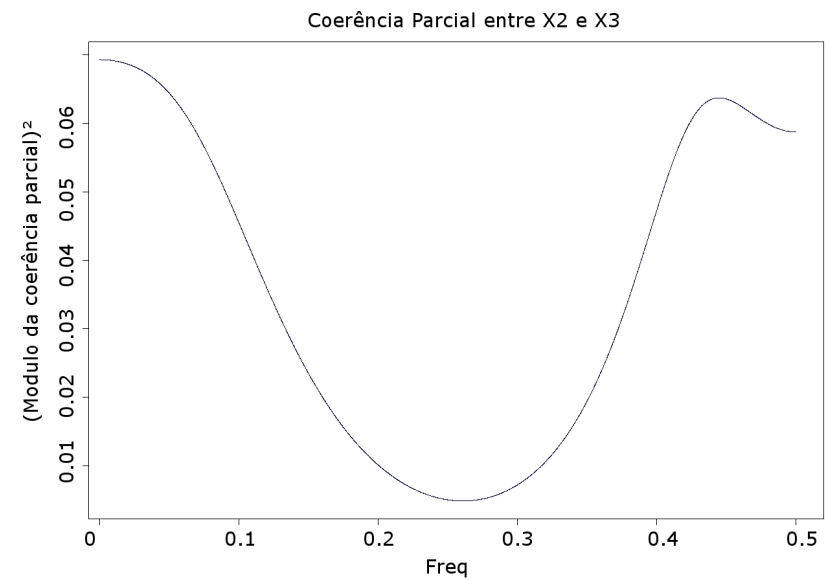

Figura 5.7: Módulo da função de coerência parcial entre $X_{2}(t)$ e $X_{3}(t)$.
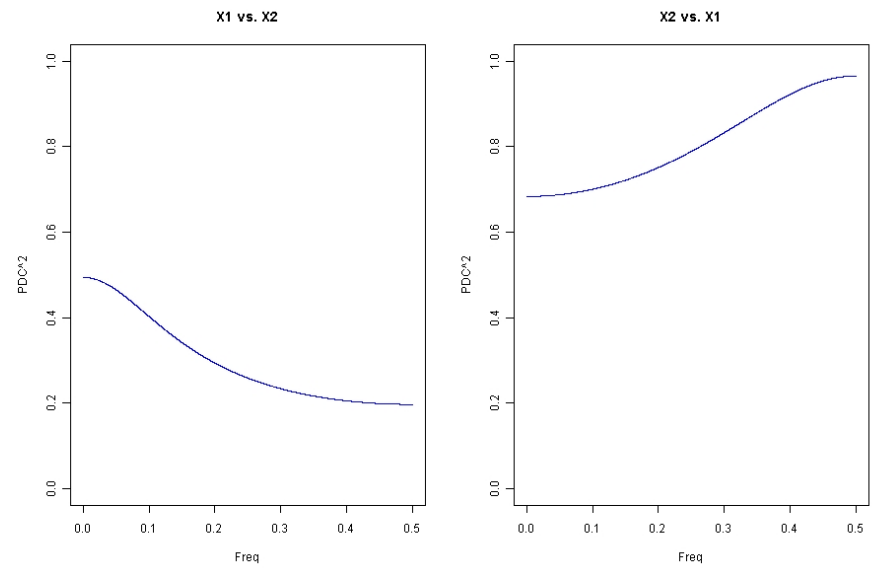

Figura 5.8: Quadrado do módulo das PDCs entre $X_{1}(t)$ e $X_{2}(t)$. 

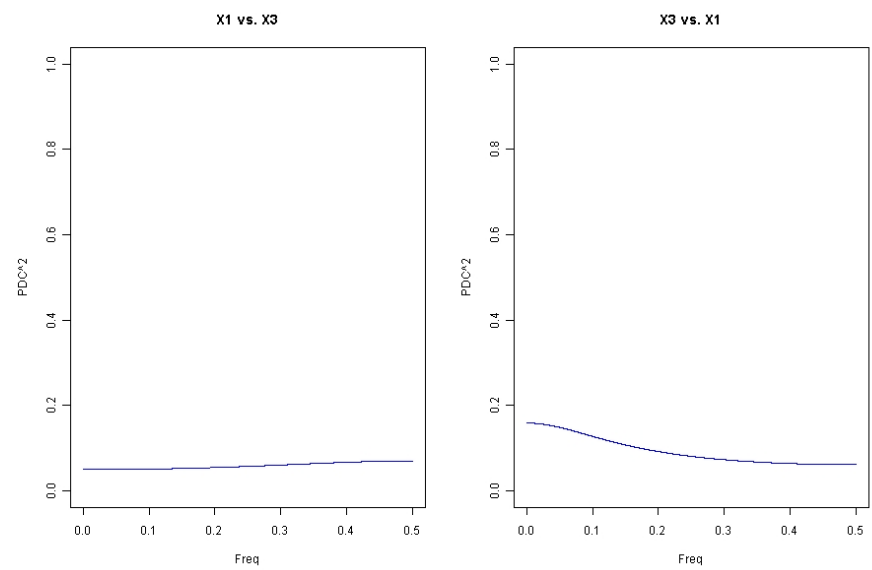

Figura 5.9: Quadrado do módulo das PDCs entre $X_{1}(t)$ e $X_{3}(t)$.
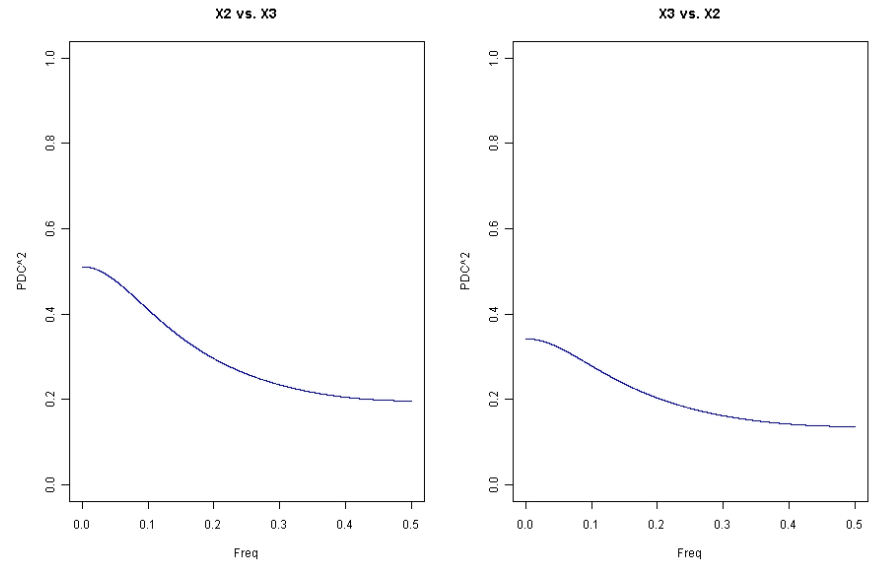

Figura 5.10: Quadrado do módulo das PDCs entre $X_{2}(t)$ e $X_{3}(t)$. 


\subsection{Resultados nos dados de EEG}

A partir de dados de EEG coletados em um indivíduo em estado de repouso com olhos fechados, com a freqüência de amostragem de $250 \mathrm{~Hz}$, temos as séries de EEG com tamanho aproximado de 200.000 observações. Foram coletadas informações de 32 canais, mas nos atentaremos à análise de 8 deles, em dois grupos distintos. As séries estudadas representam as diferenças de potencial entre o ponto de coleta do eletrodo e o ponto médio entre os eletrodos $P_{z}$ e $C_{z}$, como apresentado na figura 5.11. Analisamos a relação entre os canais F3, F4, C3 e C4 comparados dois a dois. Analogamente, foram analisados o grupo de informações coletadas nos eletrodos P3, P4, O1 e O2. A posição destes 8 eletrodos é indicada na figura 5.11 .

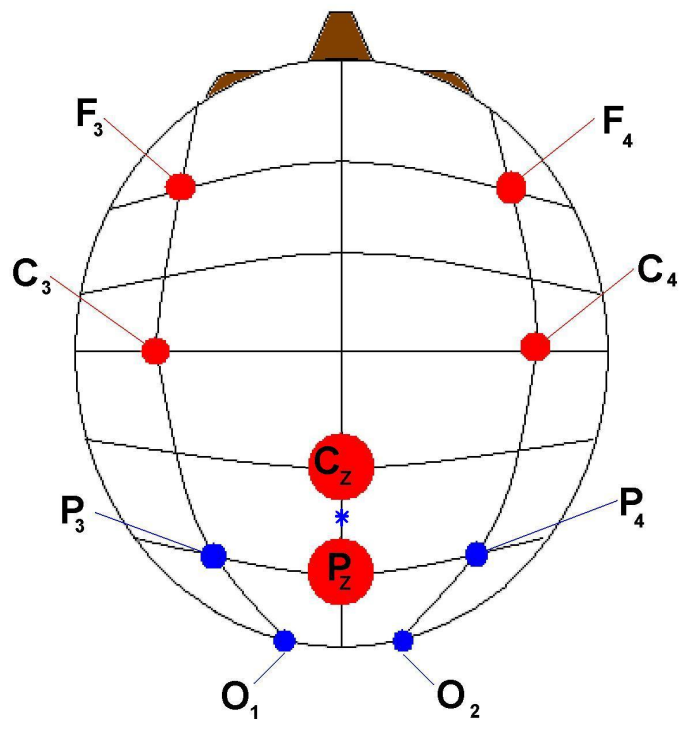

Figura 5.11: Posições dos eletrodos para coleta de EEG

Sabemos, no entanto, por conta da alta freqüência de amostragem e da grande quantidade de dados, que podemos encontrar problemas como memória longa e não-estacionariedade no estudo das séries de EEG. A figura 5.12 como evidencia tal fato. Além disso, para eliminar possíveis ruídos ocasionados pela mensuração(pensamentos involuntários do paciente, respiração, piscar de olhos, interferências no aparelho etc), optamos por aplicar um filtro "passa-banda", com a banda de filtragem de frequências entre 1 e $100 \mathrm{~Hz}$. Além disso, Bruscato(2000) sugere a construção dos estimadores de espectro com janelas móveis para evitar problemas como fuga de estacionariedade global por exemplo. Para a aplicação do filtro, utilizamos a função ffilter do pacote seewave (desenvolvido por Sueur, J. et al., 2009). A Figura 5.13 apresenta uma amostra das séries originais e das resultantes após a filtragem. Nas figuras 5.14 e 5.15 vemos os periodogramas suavizados para as séries de EEG originais. Nas figuras 5.16 e 5.17 apresentamos os periodogramas para as séries filtradas. Para ambos, foram utilizadas janelas de Daniell com tamanho 20. Notamos pouca 
mudança no periodograma em si, mas uma filtragem maior poderia comprometer a análise dos dados, já que muitas das observações de interesse ocorrem em baixas frequências. Utilizaremos ainda faixas de frequências comuns na literatura para análise de dados de EEG: Gama (frequências maiores que $30 \mathrm{~Hz})$, Beta $(13-30 \mathrm{~Hz})$, Alfa $(8-12 \mathrm{~Hz})$, Theta $(4-8 \mathrm{~Hz})$, Delta(frequências menores que $4 \mathrm{~Hz})$.
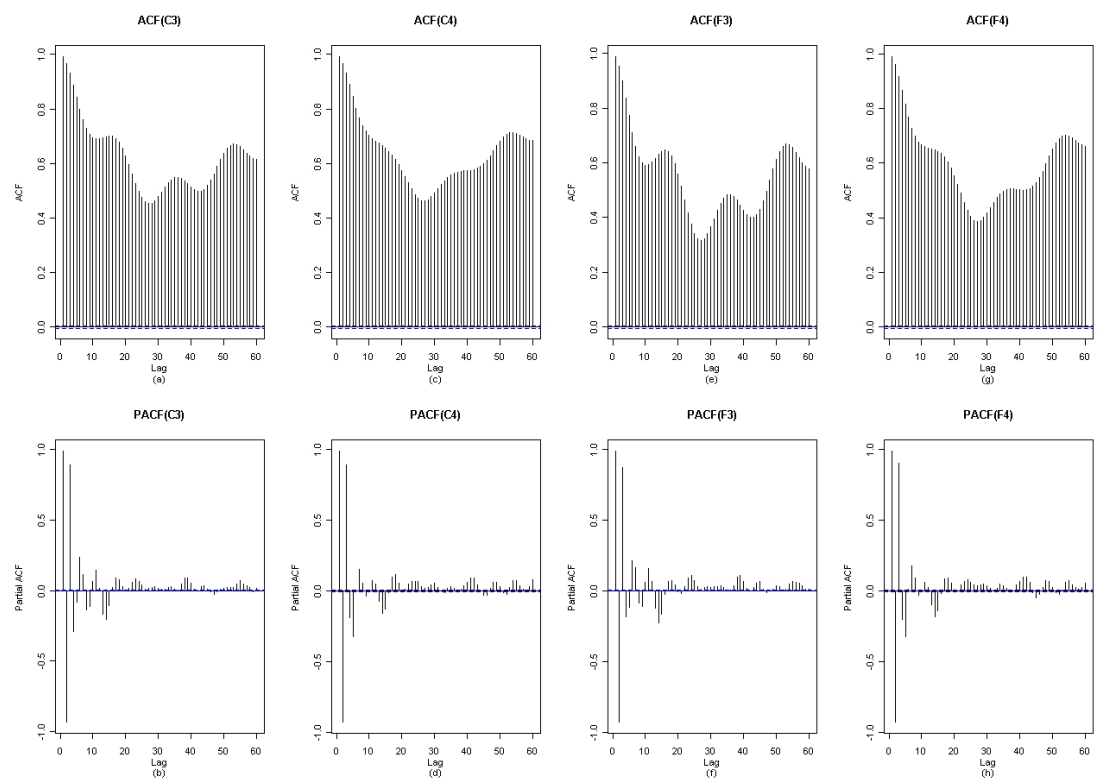

Figura 5.12: Auto-correlações e auto-correlações parciais para as séries de EEG

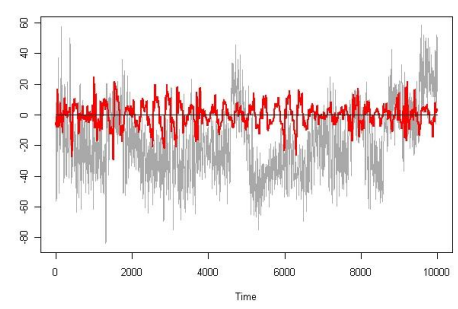

F3

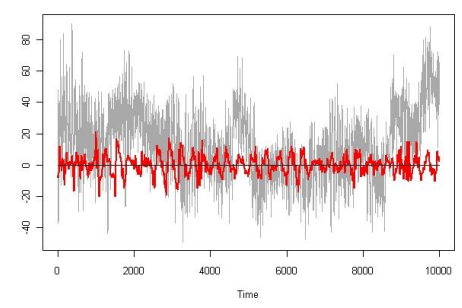

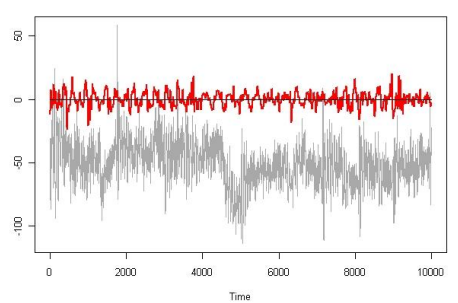

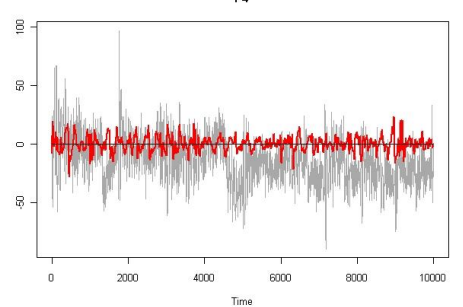

— Série Filtrada

Figura 5.13: Séries originais e resultantes filtradas 

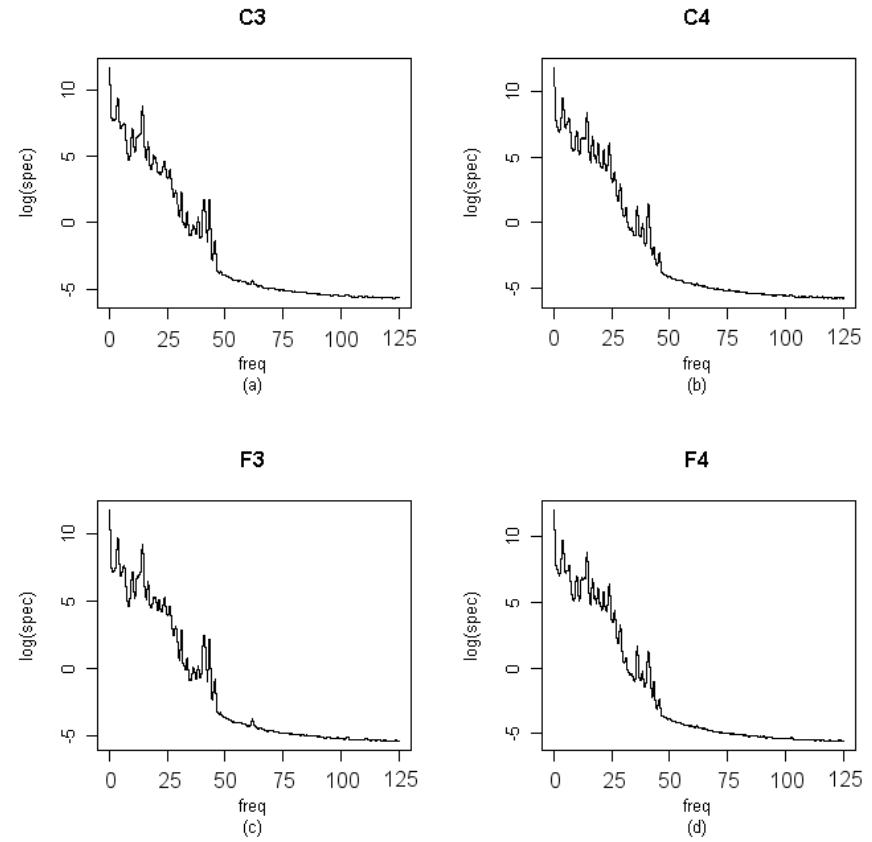

Figura 5.14: Periodogramas suavizados para as séries de EEG - Canais C3, C4, F3 e F4
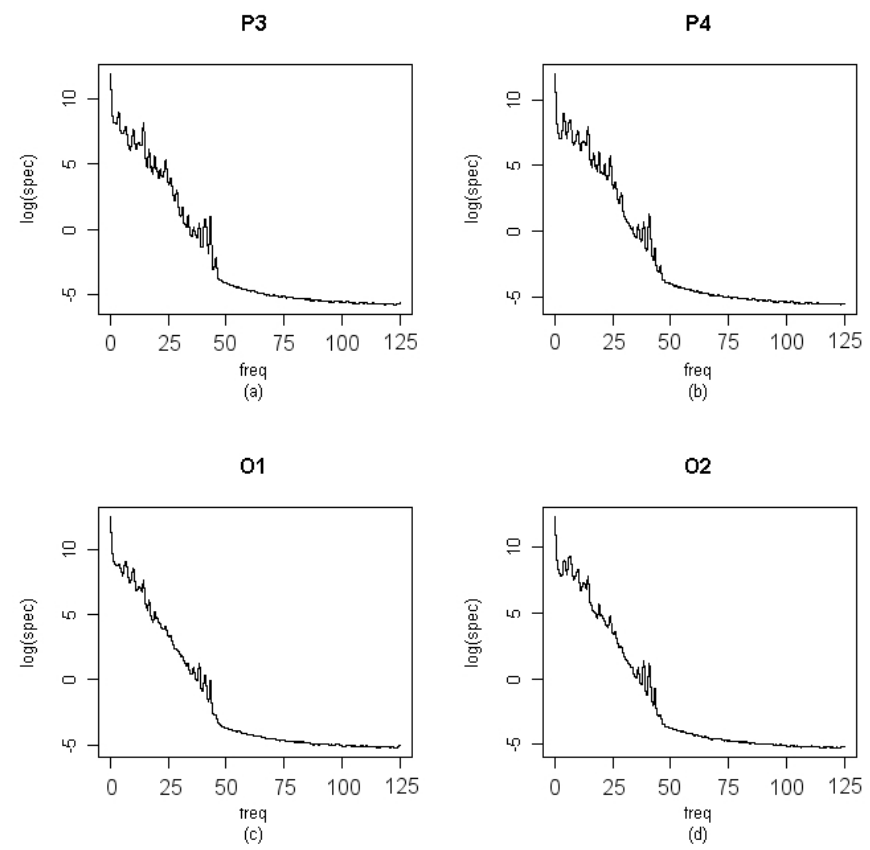

Figura 5.15: Periodogramas suavizados para as séries de EEG - Canais P3, P4, O1 e O2 

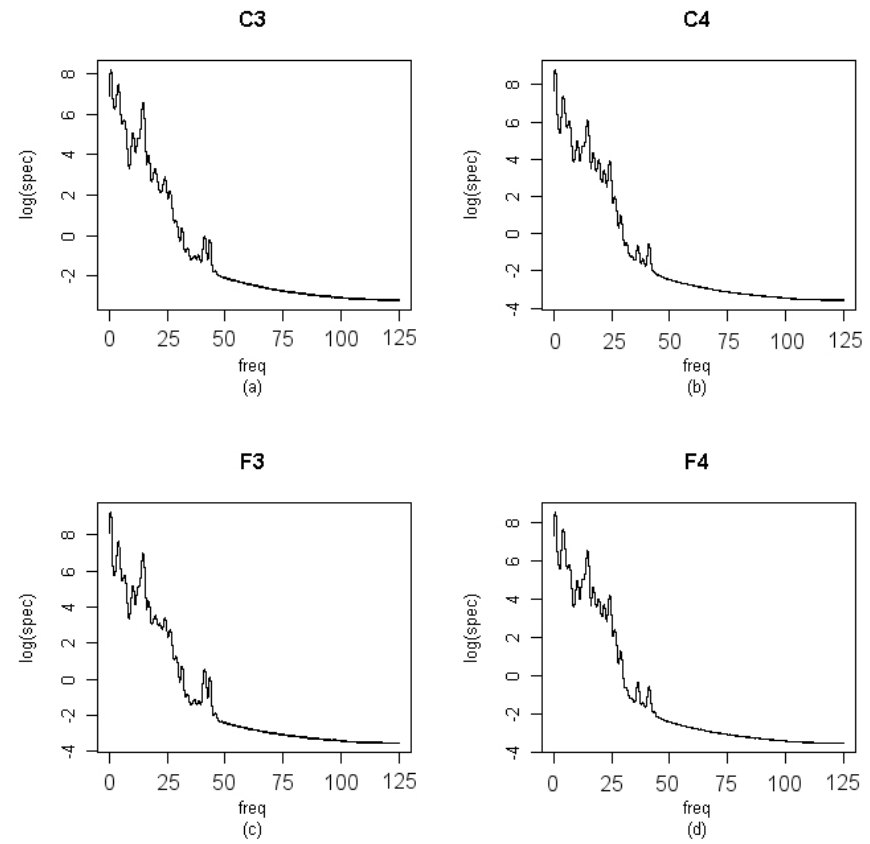

Figura 5.16: Periodogramas suavizados para as séries de EEG após a filtragem - Canais C3, C4, F3 e F4
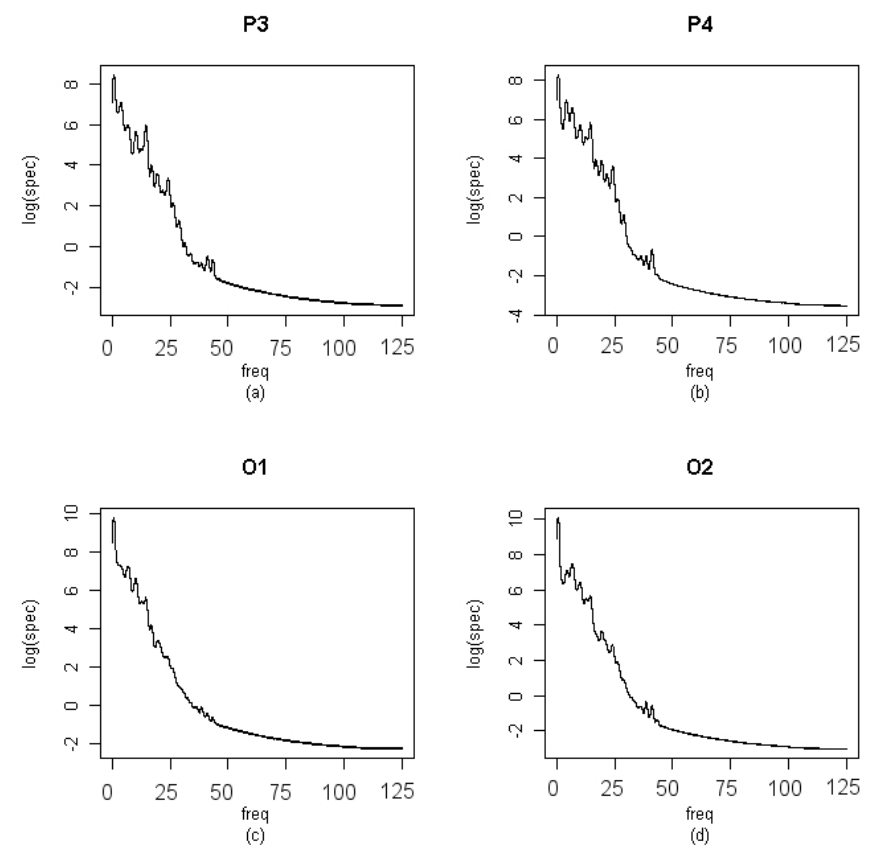

Figura 5.17: Periodogramas suavizados para as séries de EEG após a filtragem - Canais P3, P4, O1 e O2 
Construímos portanto as estimativas da função de coerência parcial utilizando janelas ao longo da série, ou seja, construímos o vetor multivariado:

$$
\begin{aligned}
\mathbf{R}_{J, Y_{a} Y_{b}, X}^{(T)}(\omega)=\left[\left.\mathbf{R}_{Y_{a} Y_{b}, X}^{(T)}(\omega)\right|_{t \in\left(J_{0}=1, J_{1}\right)}, \ldots,\right. & \\
& \left.\left.\quad \mathbf{R}_{Y_{a} Y_{b}, X}^{(T)}(\omega)\right|_{t \in\left(J_{k-1}, J_{k}\right)} \ldots,\left.\mathbf{R}_{Y_{a} Y_{b}, X}^{(T)}(\omega)\right|_{t \in\left(J_{K-1}, J_{K}=T\right)}\right]_{\left[\frac{M}{2}\right] \times K}
\end{aligned}
$$

onde $M$ é o tamanho da janela, $\left\{\left(J_{k-1}, J_{k}\right), \mathrm{k}=1, \ldots, \mathrm{K}\right\}$ é um conjunto de intervalos encaixados igualmente espaçados tal que

$$
\bigcup_{k=1}^{K}\left(J_{k-1}, J_{k}\right)=(1, T)
$$

e $\mathrm{K}=\left[\frac{T}{M}\right]$ (maior inteiro menor ou igual a $\frac{T}{M}$ ).

No Apêndice B apresentamos os gráficos de $\left|\mathbf{R}_{J, Y_{a} Y_{b}, X}^{(T)}(\omega)\right|$ (espectrogramas) para as séries de EEG à medida que percorremos as séries com a janela de $\mathrm{M}=2000$ (figuras B.1 a B.6). As figuras B.7 a B.12 mostram as curvas médias dessas funções com seus desvios-padrão. Devido à grande quantidade de gráficos desta seção, optamos por apresentar no corpo do trabalho apenas os mais relevantes. Todos os gráficos de coerência parcial e PDC encontram-se on Apêndice A. Tanto nos modelos AR utilizados ao longo das janelas para estimativa da coerência parcial quanto aqueles utilizados para a estimativa da PDC foram estimados considerando o critério de AIC para a escolha da ordem do modelo, limitando a ordem do AR à um modelo auto-regressivo de décima quinta ordem.

Note que temos maior atividade nos canais C4, F4, P3, O1 e O2. Uma atividade muito grande entre as relações dos canais ocipitais era de fato esperada, como descrito em Kaminski and Blinowska(2004). 

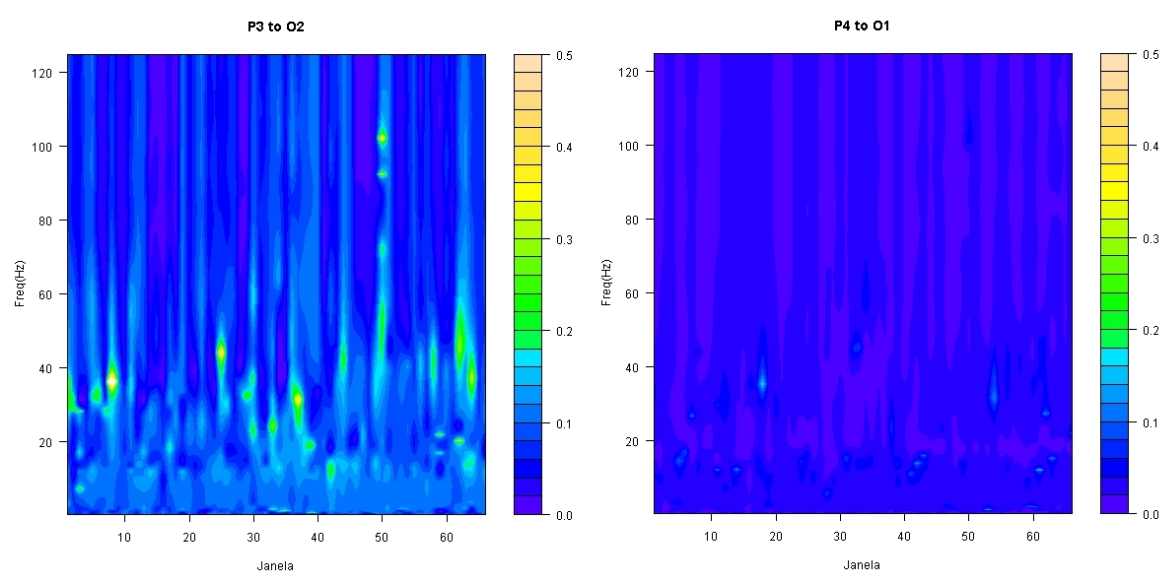

Figura 5.18: Coerência parcial com janela móvel entre os sinais P3↔O2 e P4↔O 1
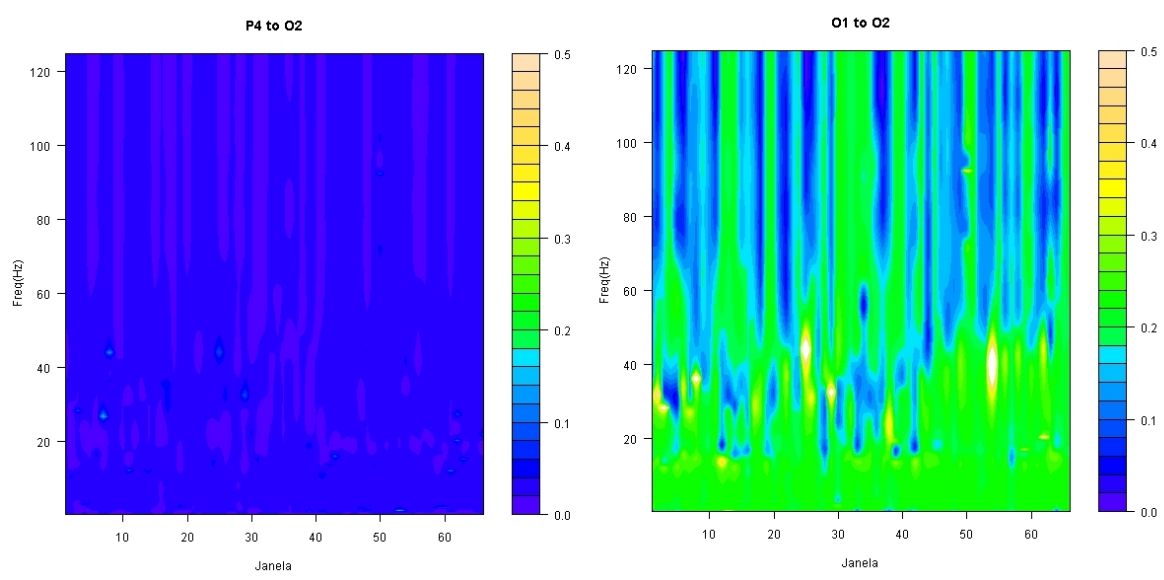

Figura 5.19: Coerência parcial com janela móvel entre os sinais $\mathrm{P} 4 \leftrightarrow \mathrm{O} 2$ e $\mathrm{O} 1 \leftrightarrow \mathrm{O} 2$

Uma das situações com menor atividade de coerência parcial ocorre quando comparamos nas relações $\mathrm{P} 4 \leftrightarrow \mathrm{O} 1$ e P4 $\leftrightarrow \mathrm{O} 2$. Vemos também que há uma grande atividade em frequências beta para as relações entre os canais ocipitais (figura 5.19).

Já quando analisamos as curvas médias, vemos que em quase todos os gráficos de coerência parcial, encontramos picos dispersão(maior variabilidade) nas frequências mais altas, acima de 30 $\mathrm{Hz}$, por vezes próximos a $40 \mathrm{~Hz}$, por outras próximos a $55 \mathrm{~Hz}$. Uma exceção a esta regra ocorre nas relações entre canais $\mathrm{P} 3 \leftrightarrow \mathrm{O} 1$. 

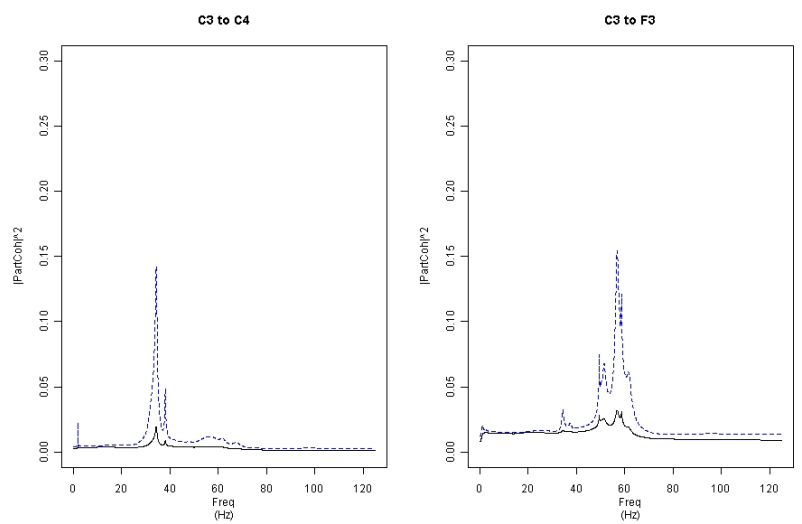

Figura 5.20: Curva média das janelas móveis das coerências parciais entre os sinais $\mathrm{C} 3 \leftrightarrow \mathrm{C} 4$ e $\mathrm{C} 3 \leftrightarrow \mathrm{F} 3$. Em preto a curva da média e em azul(tracejado) o desvio-padrão.
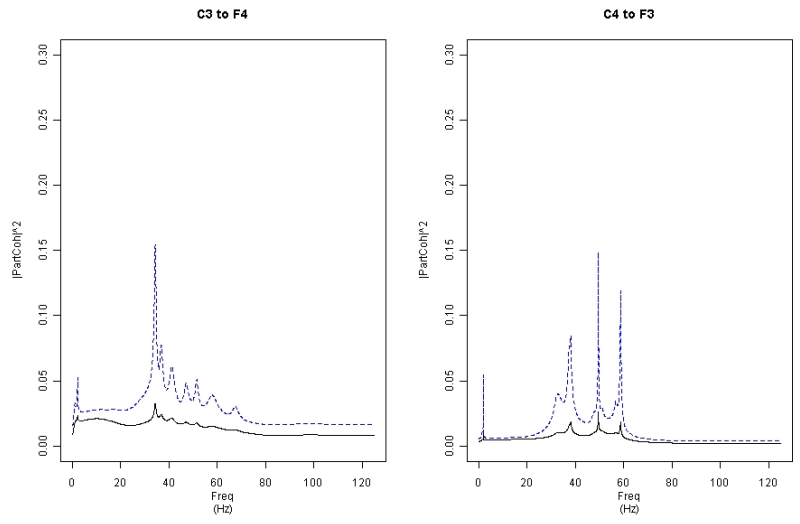

Figura 5.21: Curva média das janelas móveis das coerências parciais entre os sinais $\mathrm{C} 3 \leftrightarrow \mathrm{F} 4$ e $\mathrm{C} 4 \leftrightarrow \mathrm{F} 3$. Em preto a curva da média e em azul(tracejado) o desvio-padrão.
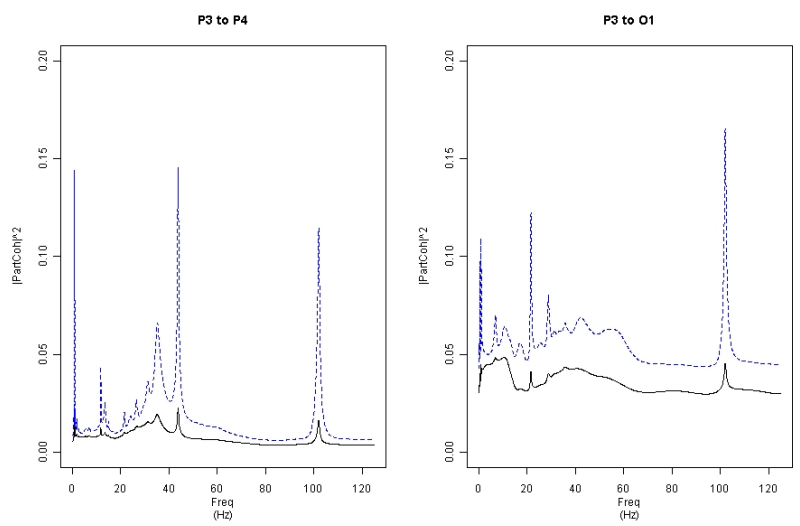

Figura 5.22: Curva média das janelas móveis das coerências parciais entre os sinais $\mathrm{P} 3 \leftrightarrow \mathrm{P} 4$ e $\mathrm{P} 3 \leftrightarrow \mathrm{O} 1$. Em preto a curva da média e em azul(tracejado) o desvio-padrão. 
Assim como as funções de coerência parcial, as funções de coerência parcial direcionada(PDC) sofrem influência de não-estacionariedade e fuga de normalidade. Utilizamos, por conta disso, as séries filtradas e construimos as funções com janelas móveis de tamanho 2000. Além disso, são ajustados modelos auto-regressivos para estimarmos a PDC em cada janela, usando como critério para a escolha da ordem, o AIC. Como este procedimento é feito de maneira automática, deve-se avaliar a qualidade dos resíduos resultantes deste ajuste. As figuras 5.23 e 5.24 apresentam os resíduos de cada série de EEG ao longo das janelas. Apresentamos assim, nas figuras 5.23 e 5.24 os níveis descritivos ( $p$-value)para o teste de Shapiro-Wilk de normalidade dos resíduos. Vemos que na maioria das estimativas das janelas, temos níveis descritivos maiores que $5 \%$, o que sugere um bom ajuste dos modelos AR em questão.

As figuras B.13 a B.24 mostram, para cada par de séries, a evolução do quadrado dos módulos das coerências parciais direcionadas ao longo das janelas. As figuras de B.25 a B.36 apresentam a curva média e o desvio-padrão dentro das janelas que compõem as figuras B.13 a B.24. Foram plotados os quadrados dos módulos das PDCs pois podemos interpretá-los como o percentual de informação que sai da série de origem para a série de destino. Para o primeiro grupo de canais, os casos em que mais notamos troca de informação ocorrem entre os canais $\mathrm{F} 3 \rightarrow \mathrm{C} 3, \mathrm{~F} 3 \rightarrow \mathrm{C} 4, \mathrm{~F} 4 \rightarrow \mathrm{C} 4$, F3 $\rightarrow$ F4. Já no grupo de canais ocipitais e parietais, vemos mais energia nas PDCs de P3 $\rightarrow$ P4, $\mathrm{P} 3 \rightarrow \mathrm{O} 2$.
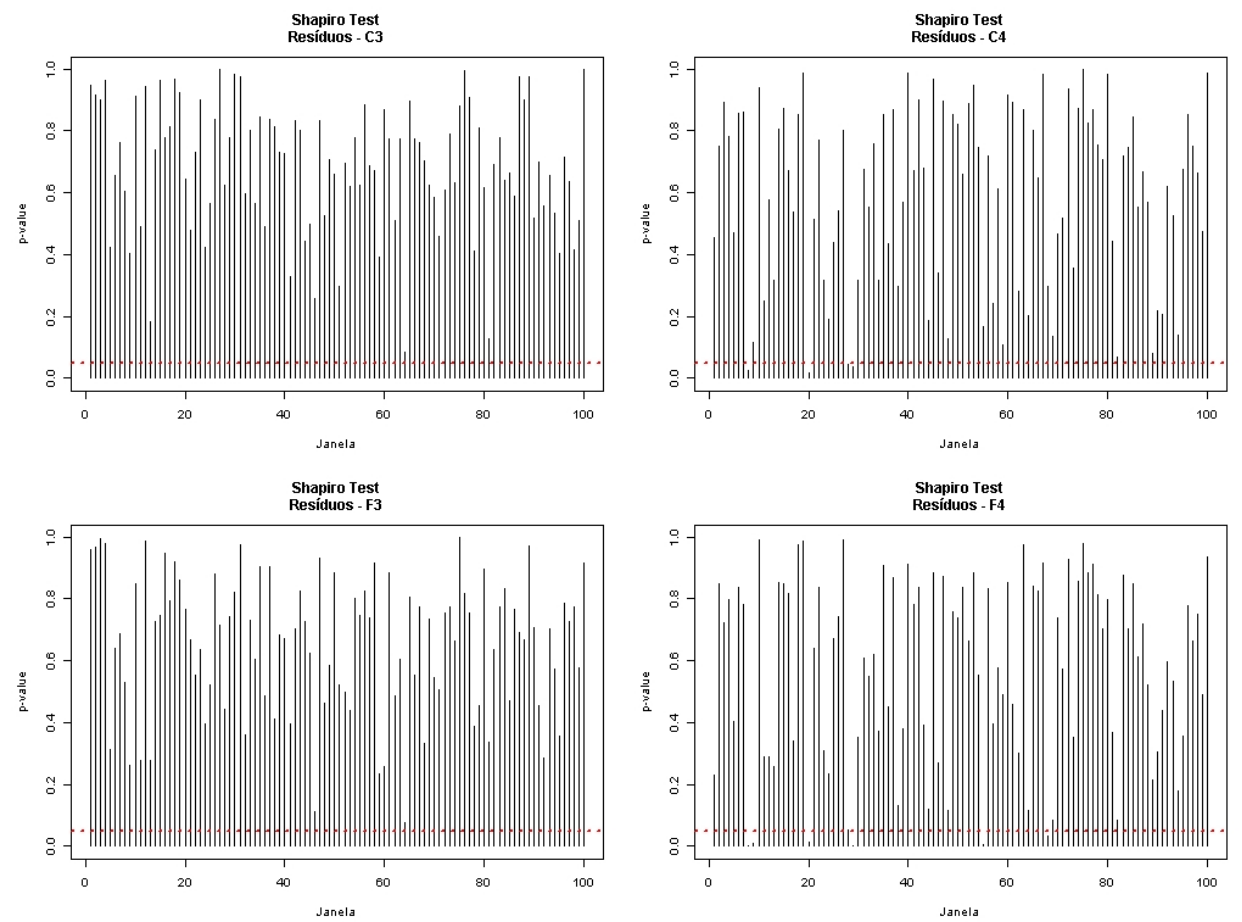

Figura 5.23: Testes de normalidade para os resíduos ao longo das janelas - eletrodos centrais (C3 e C4) e frontais $(\mathrm{F} 3$ e F4) 

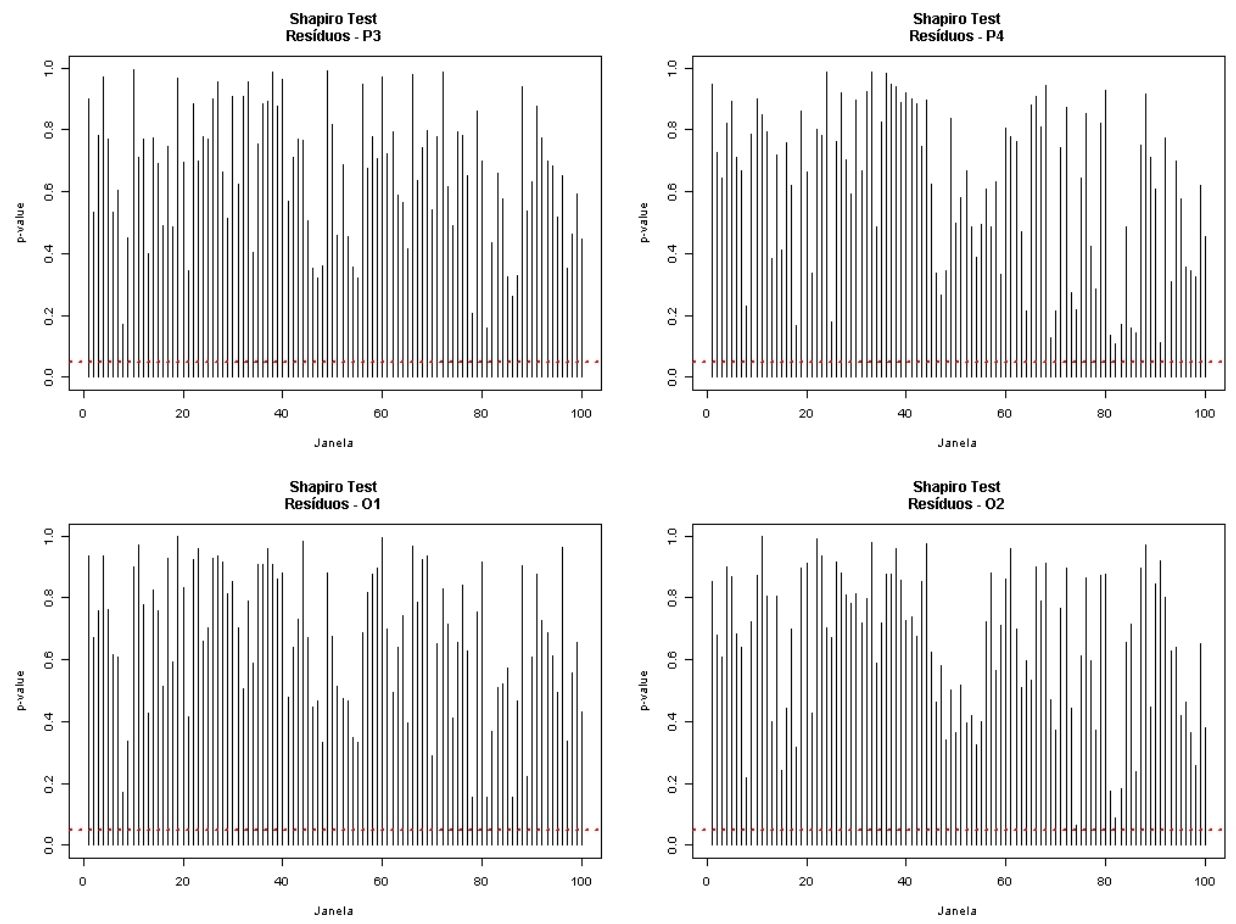

Figura 5.24: Testes de normalidade para os resíduos ao longo das janelas - eletrodos parietais (P3 e P4) e ocipitais (O1 e O2)

Para as funções de PDC(figuras B.13 a B.36 do Apêndice B), notamos que há diversos casos em que há fluxo de informação em frequências beta. Na figura 5.25 vemos que o eletrodo C3 é um ponto de constante recepção de informação em frequências beta, com origem nos canais frontais. Chama ainda atenção a troca de informações (figura 5.26)entre canais parietais (P3 e P4), e canais P3 e O1, algo já identificado nas análises de coerência parcial. Vemos no entanto que a origem do sinal ocorre no eletrodo P3 para os demais. Na figura 5.27 apresentamos casos em que identificamos picos de energia em frequências alfa nos sentidos $\mathrm{P} 3 \rightarrow \mathrm{O} 2$ e $\mathrm{P} 4 \rightarrow \mathrm{O} 1$.
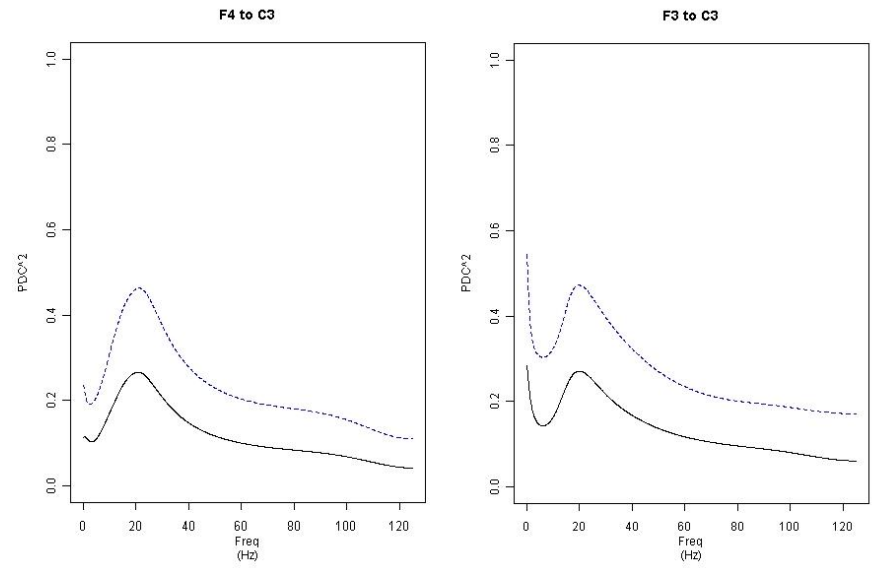

Figura 5.25: Coerência parcial direcionada com janela móvel entre os sinais F3 $\rightarrow$ C3 e F4 $\rightarrow$ C3 

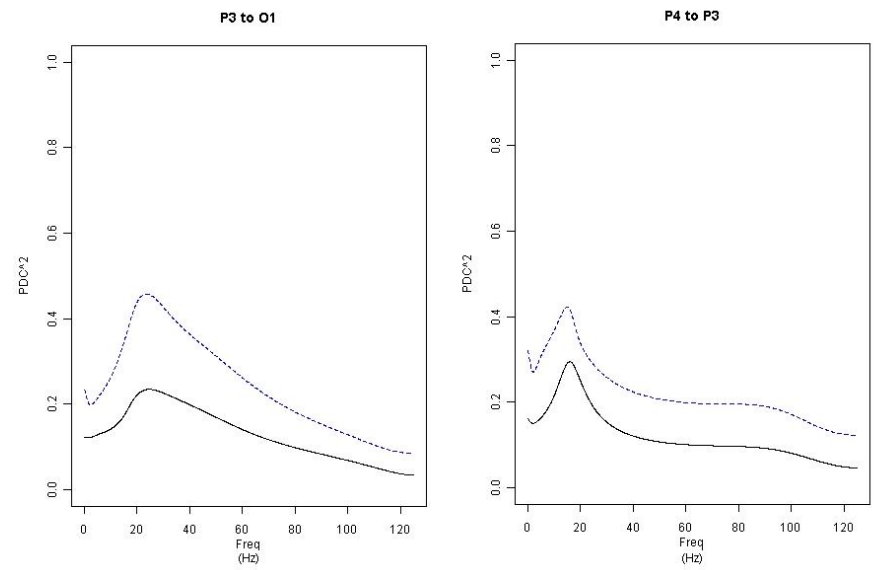

Figura 5.26: Coerência parcial direcionada com janela móvel entre os sinais P3 $\rightarrow$ P4 e P3 $\rightarrow$ O1
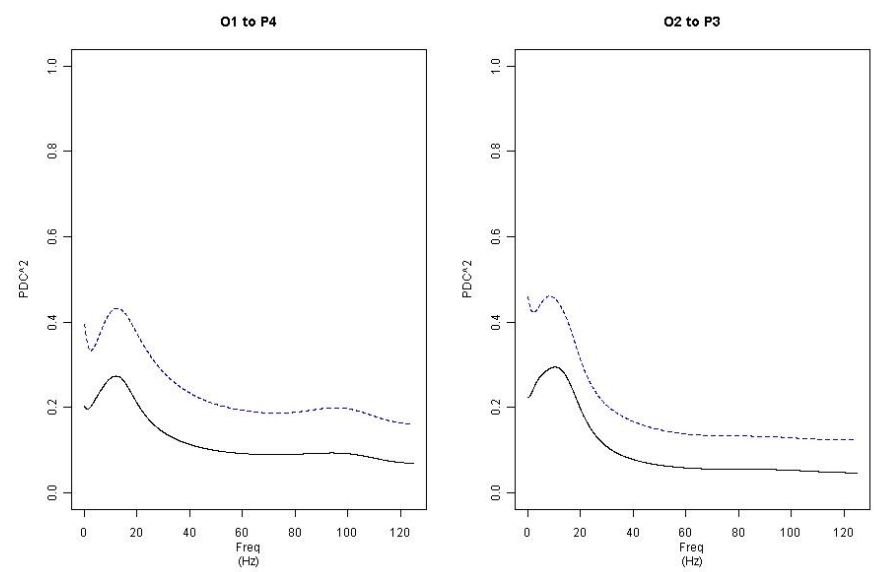

Figura 5.27: Coerência parcial direcionada com janela móvel entre os sinais $\mathrm{P} 3 \rightarrow \mathrm{O} 2$ e $\mathrm{P} 4 \rightarrow \mathrm{O} 1$

\subsection{Discussão}

As funções de correlação e coerência usuais foram e são largamente utilizadas como ferramentas para a interpretação da relação entre variáveis. Todavia, há muitas limitações quanto à interação entre variáveis. Uma de suas limitações, por exemplo, é avaliar o sentido ao qual o fluxo de informações percorre. Isto acaba por tornar difícil a interpretação dos resultados, já que não há informação sobre uma possível direção na relação entre as variáveis estudadas. Outra limitação se dá quando consideramos que outras variáveis podem influenciar na relação das variáveis estudadas. Isto pode ocasionar em falsas conclusões sobre a relação entre as variáveis de interesse.

Utilizando funções de coerência direcionada (Saito and Harashima, 1981) e coerência parcial direcionada, é possivel entender não somente o comportamento de sincronia mútua entre as séries, mas também os aspectos de influência da primeira série na segunda e vice-versa. Apesar de não termos uma relação de causalidade comprovada quando aplicamos as funções DC e PDC, temos a 
sugestão de que essa relação de fato existe. Podemos, a partir daí, construir testes de hipóteses e avaliar a relação entre as variáveis(veja Takahashi et al., 2007).

Particularmente em dados de EEG e fMRI, são estudadas a conectividade funcional e estrutual de diferentes regiões do cérebro que possuem funções e atribuições distintas. As análises de tomografia computadorizada e ressonância magnética são exames de caráter morfológico(ou estrutural) ao passo que nos dados de EEG temos uma análise funcional(quantitativa) dos dados. De uma forma ou de outra, torna-se essencial a assimetria - inequivalência nos sinais de 'ida' e 'volta' nas relações entre variáveis para o correto entendimento das relações entre módulos neuronais de encéfalo. É preciso identificar a origem da informação, o solicitante da tarefa, assim como o seu receptor, executor da tarefa.

Vemos, por exemplo, em Kus et al.(2004), uma outra análise de dados de EEG através de funções semelhantes à PDC, na qual o autor traz à discussão a importância de identificar a origem do sinal em uma relação de causa-efeito. Neste contexto, temos a PDC como uma importante ferramenta na análise de dados multi-canal, já que sua assimetria na estimação do fluxo de informação trocada pelos canais é interpretada como o percentual de informação que sai do canal de origem e vai para o canal de destino.

Por outro lado ainda, supondo que nosso processo multivariado seja um VAR de ordem finita, aspectos computacionais trazem à frente a função de coerência parcial direcionada em relação à função de coerência direcionada, já que a matriz $\mathbf{H}($.$) dada em 4.4$ não necessita ser calculada para cada frequência de interesse. A não dependência da PDC desta matriz $\mathbf{H ( . )}$ implica numa maior estabilidade da função calculada, já que, do ponto de vista computacional, a inversão da matriz de coeficientes pode apresentar problemas de singularidade, por exemplo, em casos de coeficientes estimados com amostras pequenas. Em determinados momentos nos quais se vê a necessidade de estudar o efeito isolado de dois canais, eliminando (ou ao menos reduzindo) a influência dos demais canais de estudo, vemos novamente a vantagem em se utilizar a função de coerência parcial direcionada. Isto não é, no entanto, válido se tivermos um processo com estruta de médias móveis multivariadas (VARMA com coeficientes autoregressivos nulos), já que sua representação como VAR se dá com um VAR de ordem infinita.

Ginter Jr. et al.(2005) e Biswal et al.(1995) descrevem a relação entre regiões pré-motoras (regiões frontais, F3 e F4) e motoras (canais centrais, canais C3 e C4, por exemplo) durante a execução de movimentos(ou de movimentos imaginários). Neste contexto, vemos nas figuras B.14 a B.17 nítida troca de informações entre os canais frontais e centrais em frequências beta, com origens nos canais frontais. Vemos também relação inter-hemisférios de F3 para F4, como na figura B.18, também em frequências beta. Biswal et al.(1995) e van den Heuvel et al.(2009) descrevem, do ponto de vista médico, estas relações entre regiões motoras e pré-motoras a partir de dados 
de fMRI(functional Magnetic Resonance Imaging). Picos existentes em maiores frequências, como evidentes na figura B.14, C3 $\rightarrow$ F3, são resultado de ruídos eventualmente não removidos nas séries, durante a filtragem.

A partir dos resultados obtidos mensurando indivíduos no mesmo estado de estímulo - ou seja, em repouso, com olhos fechados - Kaplan and Shishkin(2000) concluíram sobre a relação significativa entre os eletrodos de posição F3, F4, C3 e C4 assim como entre os eletrodos nas posições P3, $\mathrm{P} 4$, O1 e $\mathrm{O} 2$ formando duas redes de relacionamento entre canais. Em seu trabalho, Kaplan and Shishkin(2000) utilizaram técnicas não paramétricas (teste de Wilcoxon pareado, n=12) para comparar a relação entre os canais.

Concluimos, porém, neste trabalho, não apenas sobre as relações entre estes canais, mas também sobre qual canal é o 'originário' da informação e qual o canal 'receptor'. Em outras palavras, damos a idéia não só de direção da informação, mas também o sentido percorrido por ela - assim como em quais frequências estas relações se apresentam mais fortes.

Se analisarmos as figuras de PDC para a rede de ligações entre canais parietais e ocipitais, encontraremos relações semelhantes àquelas descritas por Kus et al. (2004). Vemos uma forte transferência de informação com saída nos canais ocipitais para os canais parietais, notademente nas figuras B.21, B.22 e B.23 em faixas de frequência alfa. Note que a volta não ocorre com a mesma intensidade. Temos apenas na figura B.20 um grande fluxo informação saindo de P3 para O1, em frequências beta e gama. Estas relações entre canais ocipitais e parietais(veja Van den Heuvel, 2009) são conhecidas na literatura como core networks, e são aquelas que controlam a visão e a atenção sensorial. Já as relações entre regiões posteriores com regiões mediais-frontais são denominadas de default mode networks e controlam movimentos musculares voluntários, como descrito em Biswal et al.(1995) e Ginter et al.(2005).

Encontramos ainda diversas relações entre canais inter-hemisférios. Vemos na figura B.19 picos de energia em frequências beta e gama para a relação $\mathrm{P} 3 \rightarrow \mathrm{P} 4$, ao passo que vemos a relação P4 $\rightarrow$ P3 concentrada ao redor das frequências de faixa alfa e beta. Para os canais ocipitais, os quais têm PDC dadas na figura B.24, vemos uma maior concentração de saída de informação de O2 para $\mathrm{O} 1$ do que de $\mathrm{O} 1$ para $\mathrm{O} 2$. As relações que envolvem os canais ocipitais como origem da informação mostram, em geral, picos em frequências mais baixas, em geral na faixa alfa.

As funções DC e PDC e suas aplicações possibilitaram muitos avanços não somente na teoria de séries temporais, mas também na área médica. A partir de Saito e Harashima(1981) e Baccala e Sameshima(2001), temos diversos artigos apresentando maneiras diferentes de estimar a relação direcionada entre variáveis. Temos, por exemplo a função Directed Transfer Function(DTF) em Kaminski(1991) e a função Relative Power Contribution(RPC) em Yamashita et al.(2005). A DTF 
baseia-se também em ajustes de modelos AR para estimar a propagação(direção) da informação e propriedades espectrais dos sinais investigados. Já a RPC é definida como a taxa de contribuição de cada variável(ou par de variáveis) tem sobre o espectro total do processo em estudo.

Friston et al. (2003) apresentam a idéia de modelagem dinâmica de causalidade (dynamic causal modelling - DCM). De uma maneira geral, de fato podemos concluir sobre causalidade com a DCM, o que a torna uma das técnicas mais completas neste contexto. A idéia central por trás da DCM é tratar o cérebro como um sistema determinístico não-linear dinâmico com variáveis de entrada produzindo variáveis de saída. No entanto, a DCM precisa ter indicadas previamente a existência de relação entre as variáveis. Uma outra possível forma de análise para a PDC é, por exemplo, indicar quais são essas relações prévias para a função DCM. Dessa maneira, pode-se concluir sobre a causalidade sugerida na análise de PDC. 


\section{Capítulo 6}

\section{Considerações Finais}

Neste estudo, vimos diversas funções que avaliam o grau de relação entre séries de tempo multivariadas no domínio da frequência. A mais simples de todas, a função de coerência, nada mais é do que uma função análoga à função de correlação do domínio do tempo. A função de coerência parcial mede o grau de relação entre duas séries após removidos efeitos de outras séries no processo.

Tanto na função de coerência parcial quanto na função de coerência parcial direcionada, vimos que para os dados de EEG, a alta frequência de amostragem e a grande quantidade de dados pode causar problemas como memória longa e fuga de nomalidade dos resíduos dos modelos autoregressivos multivariados, o que gera problemas sérios de estimação da coerência parcial. Como solução, utilizamos o artifício de estimar as funções de coerência parcial e PDC em janelas móveis ao longo do intervalo de observações, contruindo assim um conjunto de estimativas de funções, ordenados ao longo das janelas. A partir daí, apresentamos os gráficos das figuras B.1 a B.6.

Outra maneira de estimar a relação entre séries temporais multivariadas pode ser feito por meio de análise de ondaletas. Percival and Walden(2007) discutem extensivamente sobre essa metodologia aplicada na mensuração de relação entre séries temporais. Torrence and Compo(1998) apresentam um guia prático de aplicações de ondaletas no estudo de séries temporais multivariadas. Outras aplicações de ondaletas no estudo de interações em séries temporais podem ser vistas em Torrence and Webster (1999) e Grinsted et al. (2004). 


\title{
Apêndice A
}

\section{Sintaxe R - Funções utilizadas}

\author{
Função de Coerência Parcial
}

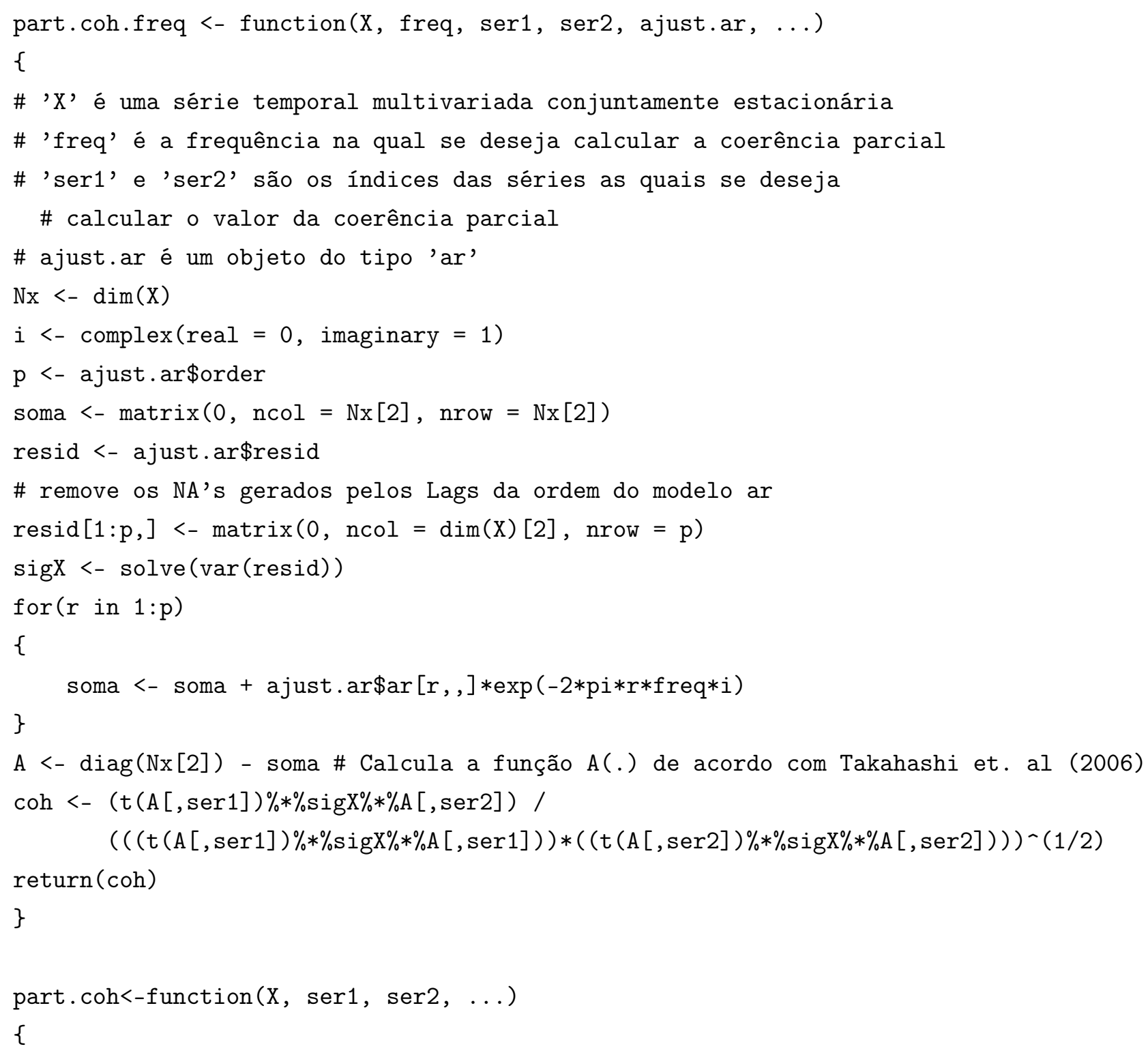




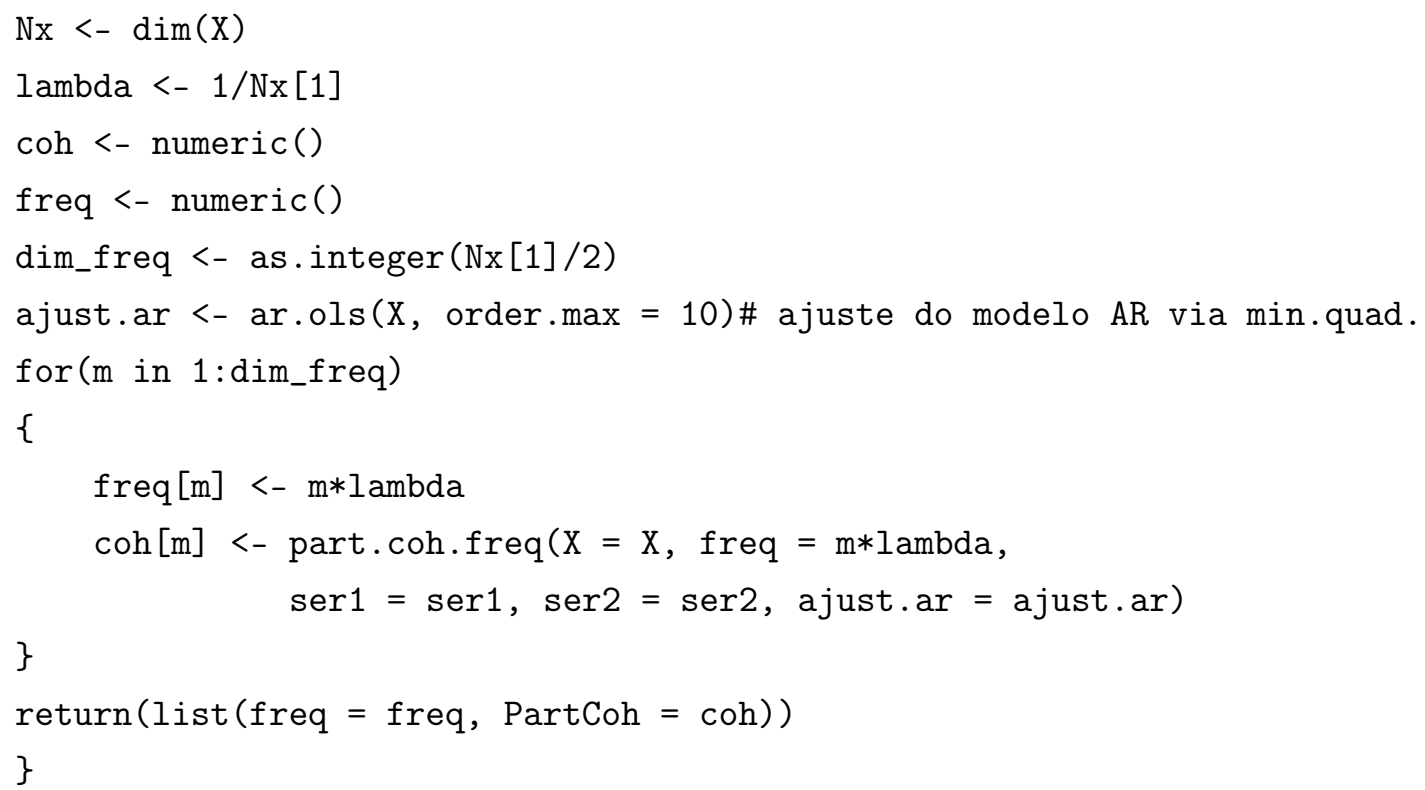




\section{Função de Coerência Parcial Direcionada}

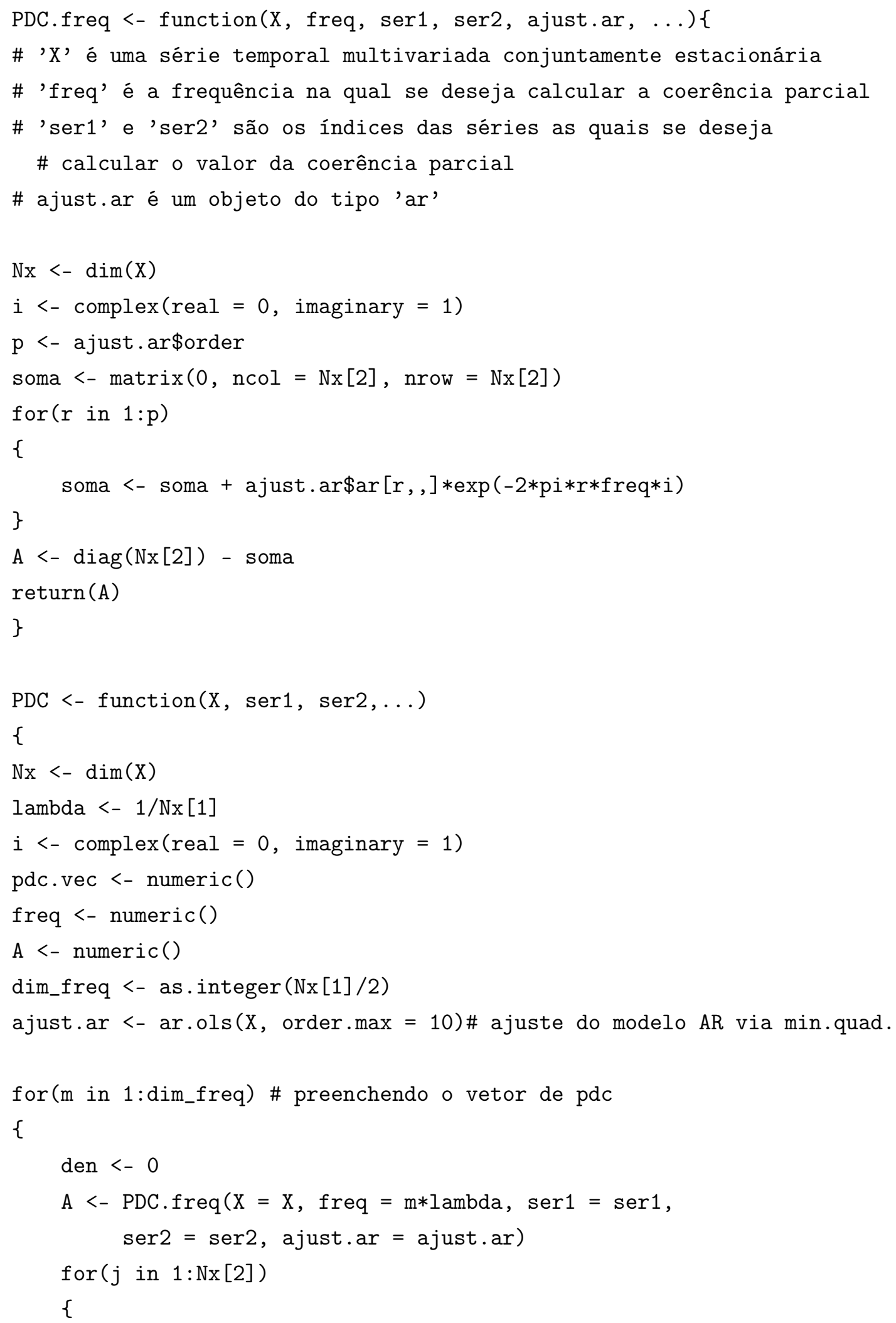




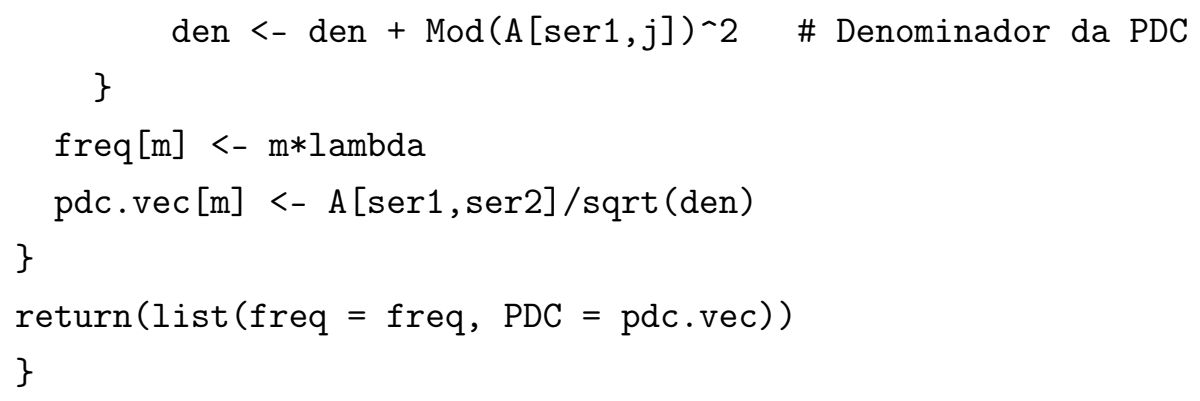

Função de Coerência Parcial com janela móvel

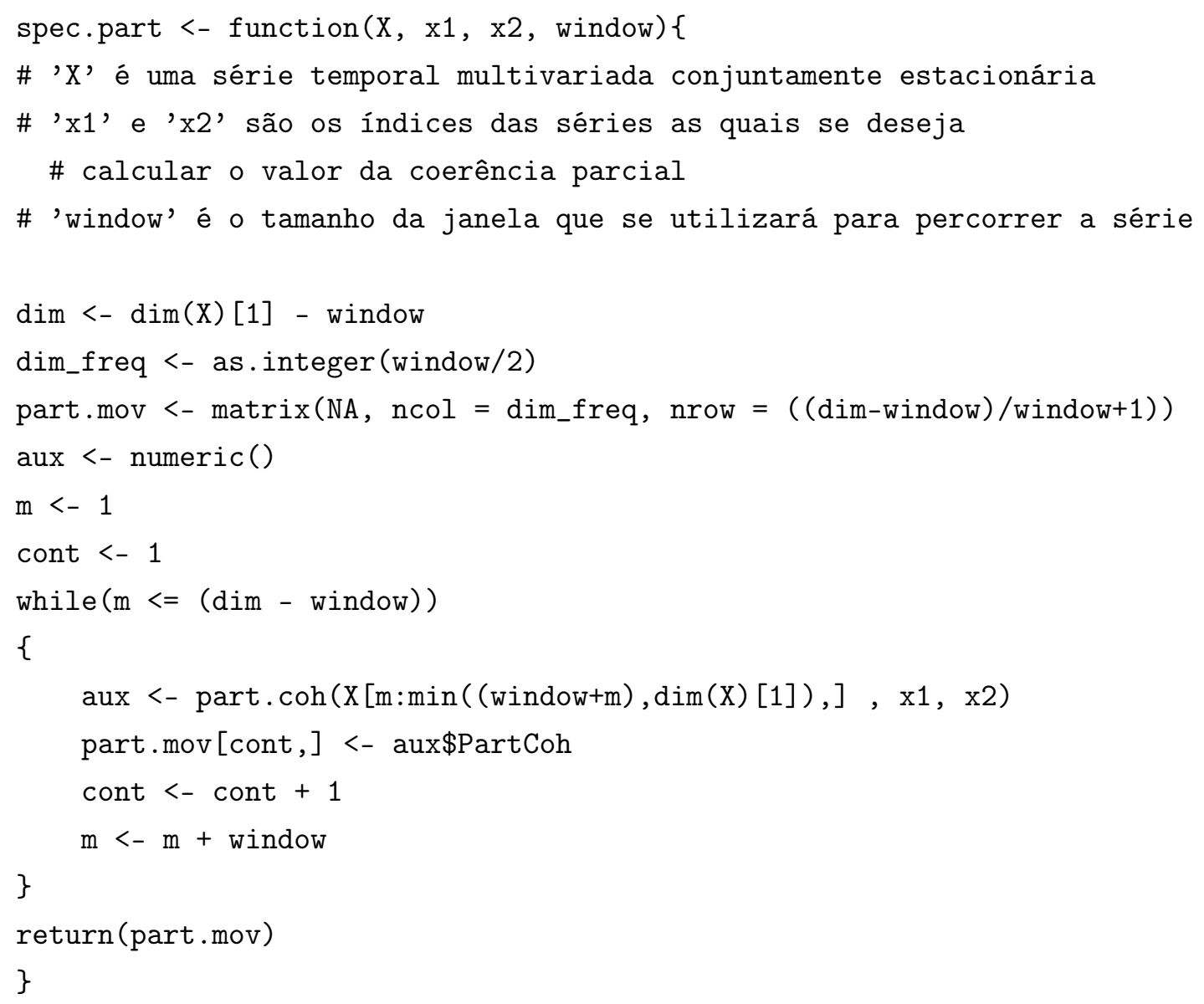

Função de Coerência Parcial Direcionada com janela móvel

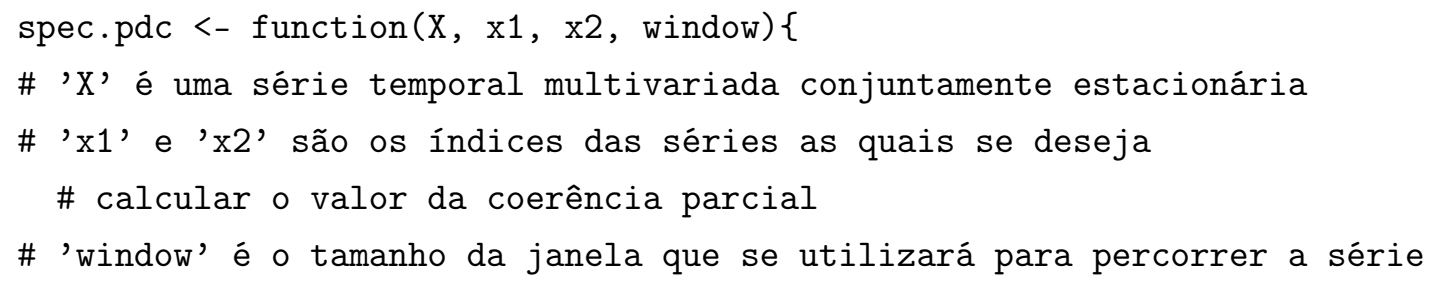




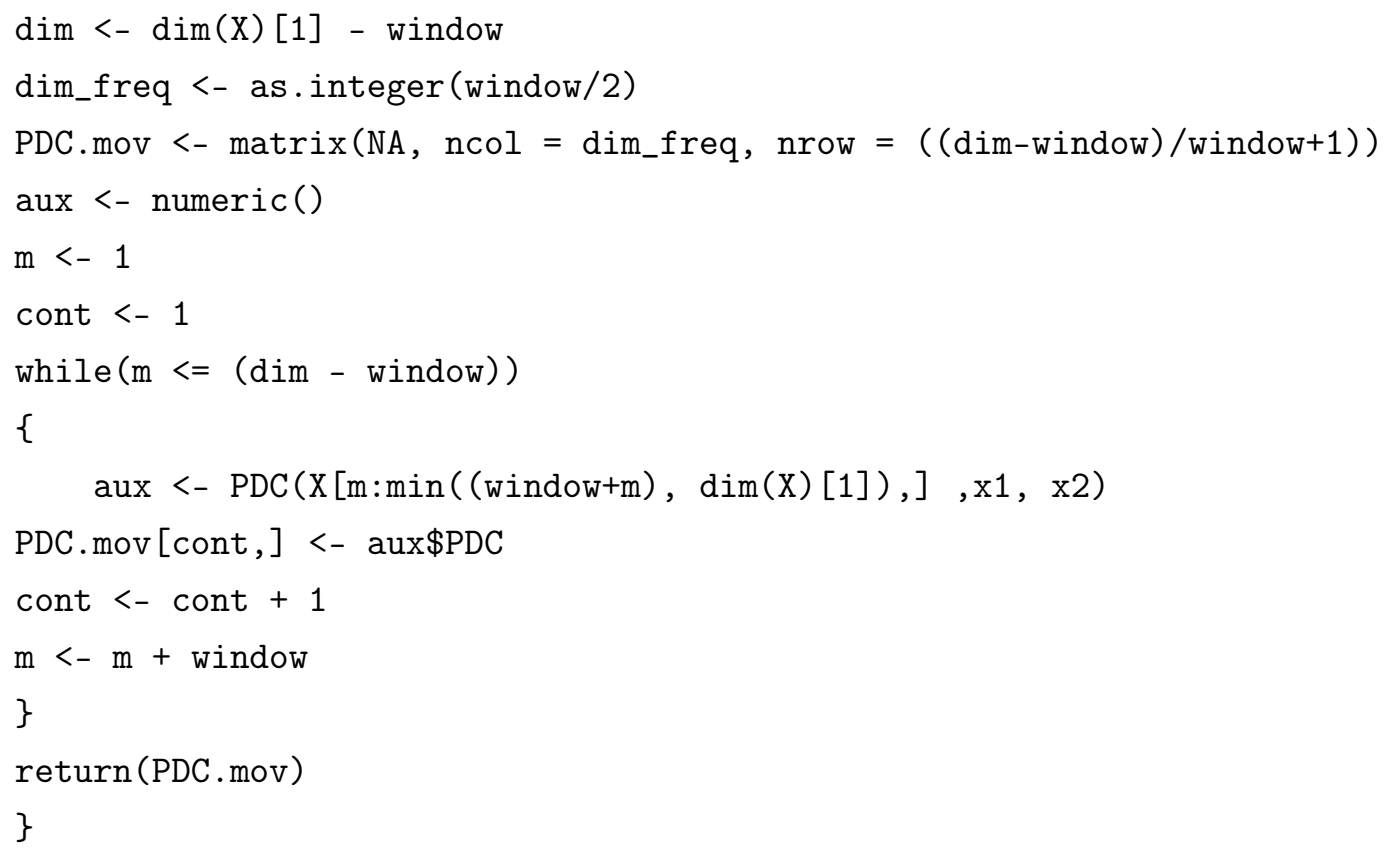




\section{Apêndice B}

\section{Gráficos e Figuras de Coerência Parcial e PDC}
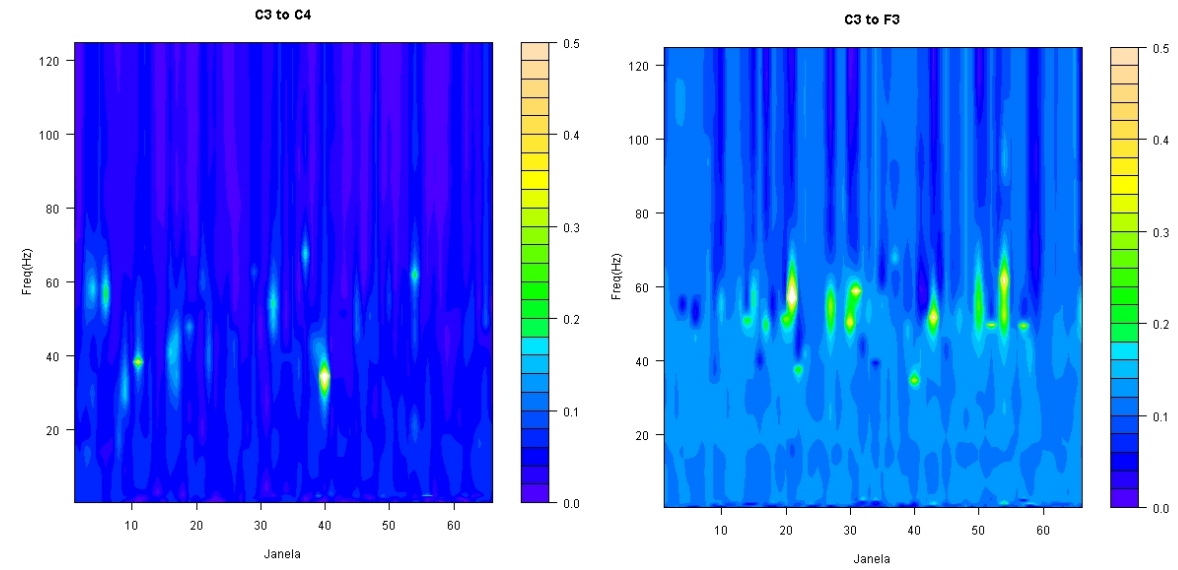

Figura B.1: Coerência parcial com janela móvel entre os sinais C3↔C4 e C3↔F3
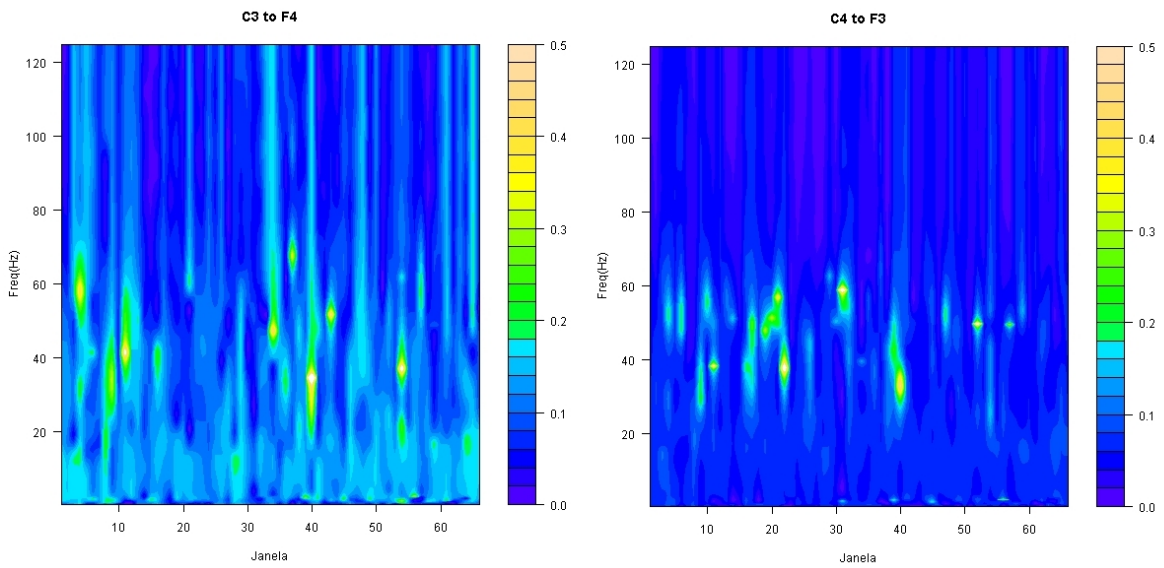

Figura B.2: Coerência parcial com janela móvel entre os sinais C3↔F4 e C4↔F3 

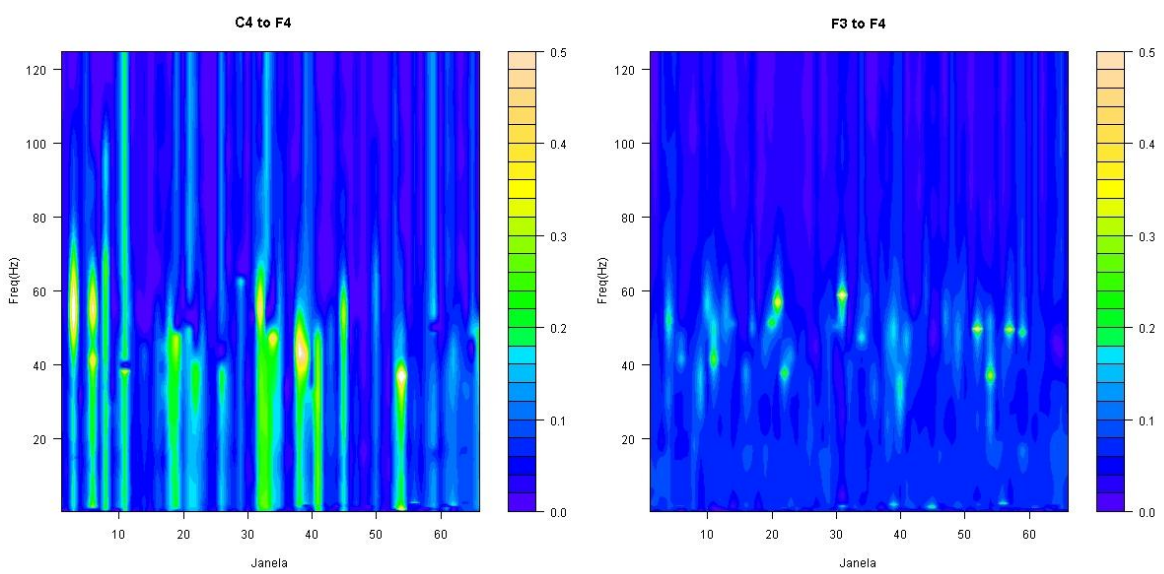

Figura B.3: Coerência parcial com janela móvel entre os sinais $\mathrm{C} 4 \leftrightarrow \mathrm{F} 4$ e F3↔F4
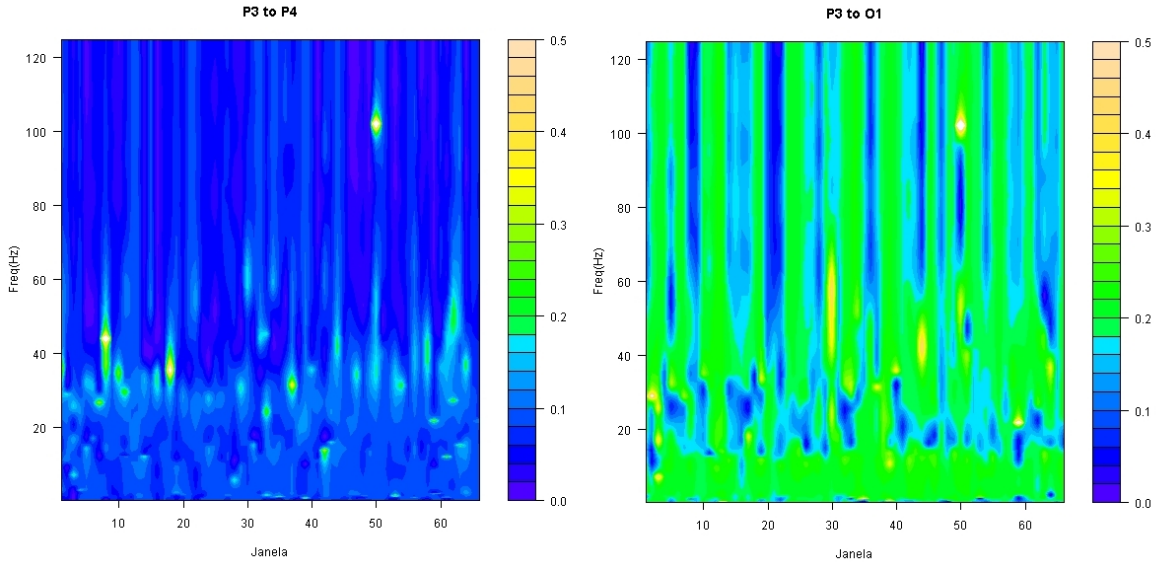

Figura B.4: Coerência parcial com janela móvel entre os sinais P3↔P4 e P3↔O1 

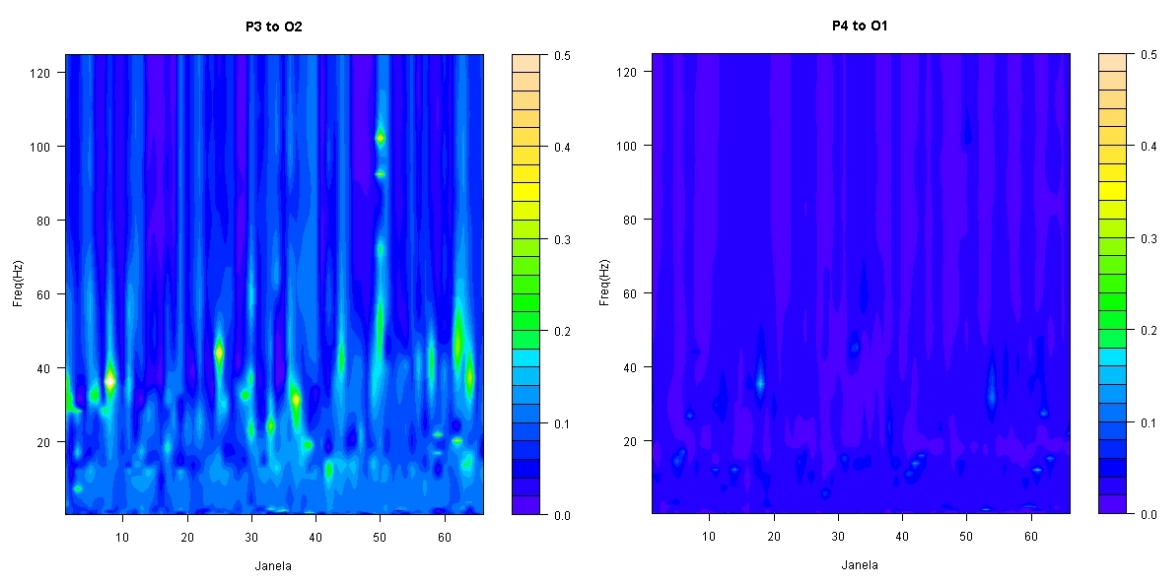

Figura B.5: Coerência parcial com janela móvel entre os sinais $\mathrm{P} 3 \leftrightarrow \mathrm{O} 2$ e $\mathrm{P} 4 \leftrightarrow \mathrm{O} 1$
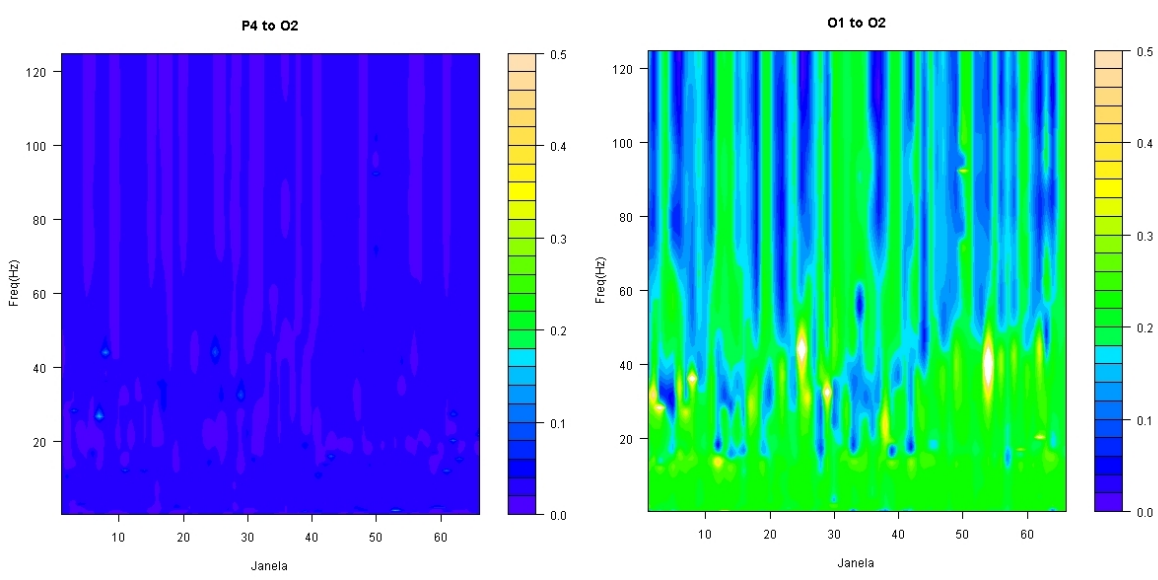

Figura B.6: Coerência parcial com janela móvel entre os sinais $\mathrm{P} 4 \leftrightarrow \mathrm{O} 2$ e $\mathrm{O} 1 \leftrightarrow \mathrm{O} 2$
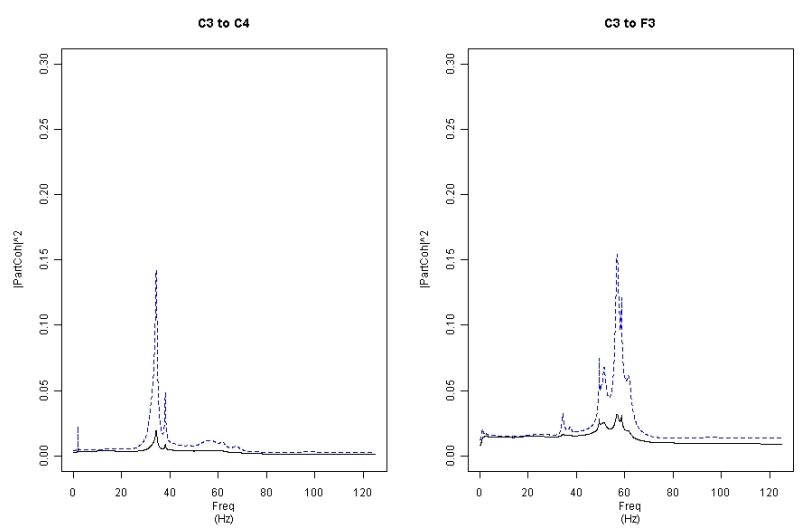

Figura B.7: Curva média das janelas móveis das coerências parciais entre os sinais $\mathrm{C} 3 \leftrightarrow \mathrm{C} 4$ e C3↔F3. Em preto a curva da média e em azul(tracejado) o desvio-padrão. 

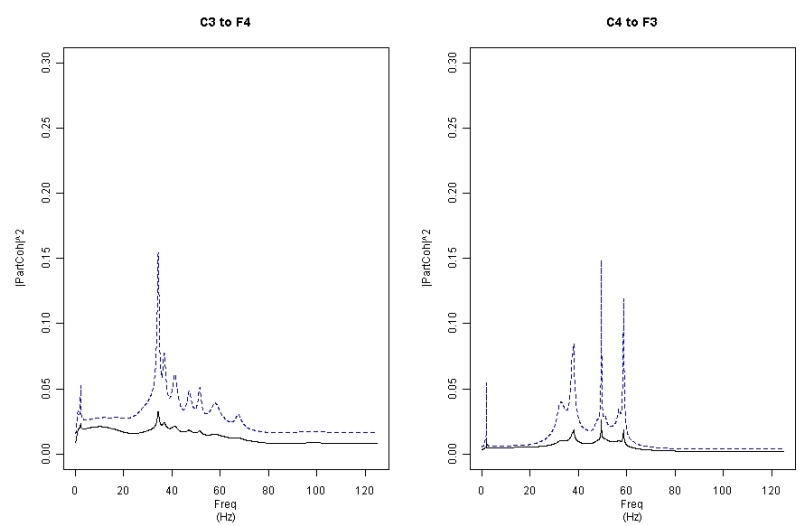

Figura B.8: Curva média das janelas móveis das coerências parciais entre os sinais $\mathrm{C} 3 \leftrightarrow \mathrm{F} 4$ e $\mathrm{C} 4 \leftrightarrow \mathrm{F} 3$. Em preto a curva da média e em azul(tracejado) o desvio-padrão.
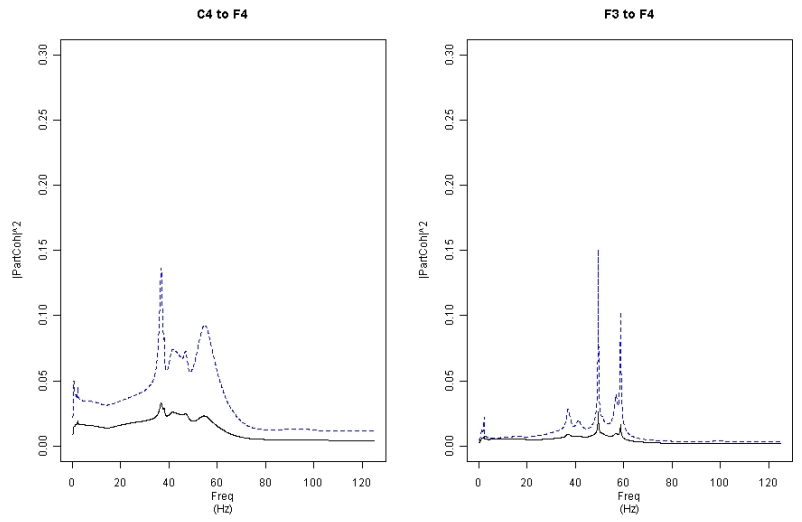

Figura B.9: Curva média das janelas móveis das coerências parciais entre os sinais $\mathrm{C} 4 \leftrightarrow \mathrm{F} 4$ e F3↔F4. Em preto a curva da média e em azul(tracejado) o desvio-padrão.
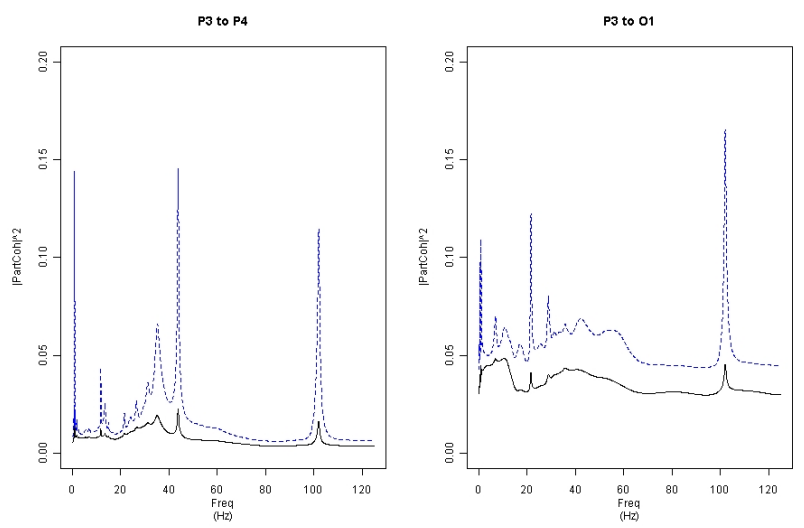

Figura B.10: Curva média das janelas móveis das coerências parciais entre os sinais $\mathrm{P} 3 \leftrightarrow \mathrm{P} 4$ e $\mathrm{P} 3 \leftrightarrow \mathrm{O} 1$. Em preto a curva da média e em azul(tracejado) o desvio-padrão. 

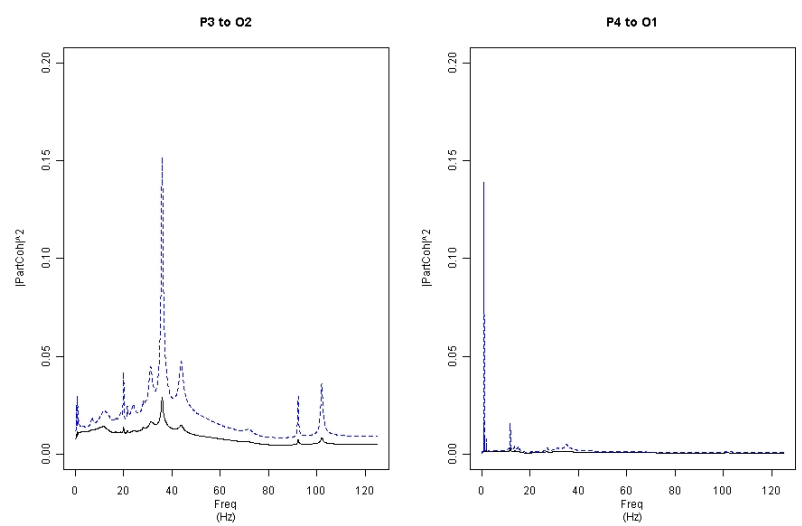

Figura B.11: Curva média das janelas móveis das coerências parciais entre os sinais $\mathrm{P} 3 \leftrightarrow \mathrm{O} 2$ e $\mathrm{P} 4 \leftrightarrow \mathrm{O} 1$. Em preto a curva da média e em azul(tracejado) o desvio-padrão.
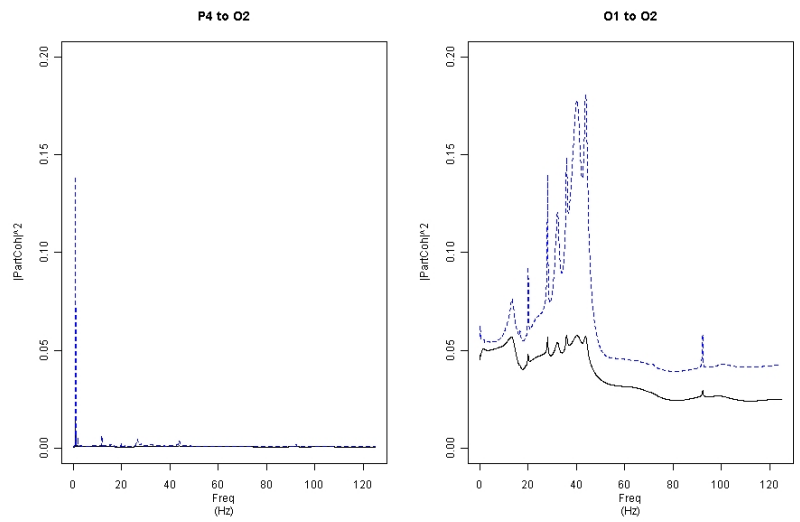

Figura B.12: Curva média das janelas móveis das coerências parciais entre os sinais $\mathrm{P} 4 \leftrightarrow \mathrm{O} 2$ e $\mathrm{O} 1 \leftrightarrow \mathrm{O} 2$. Em preto a curva da média e em azul(tracejado) o desvio-padrão.
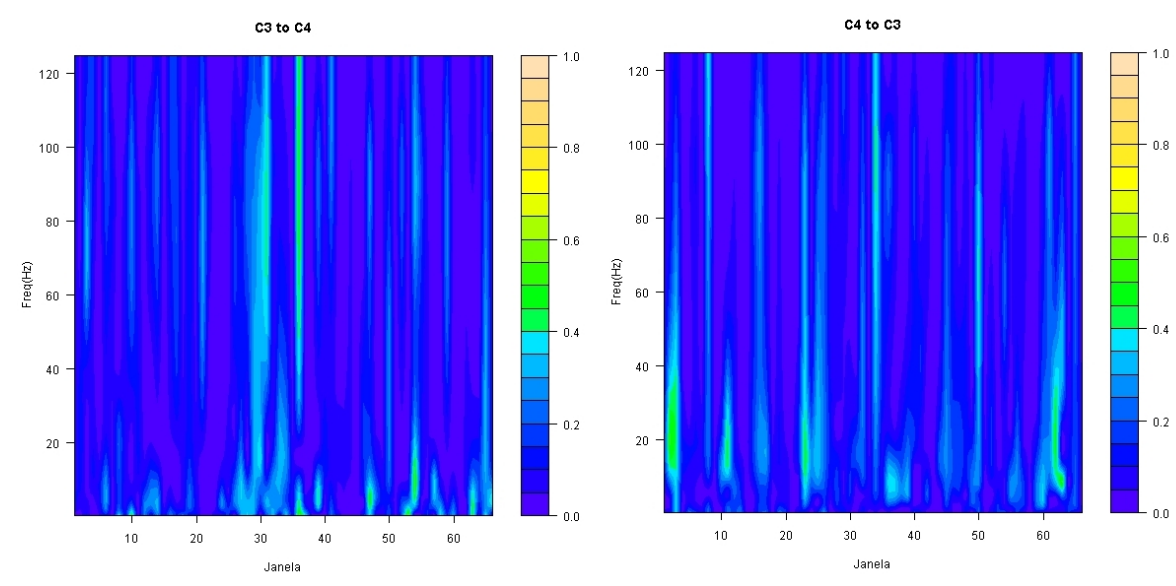

Figura B.13: Coerência parcial direcionada com janela móvel entre os sinais C3 e C4 

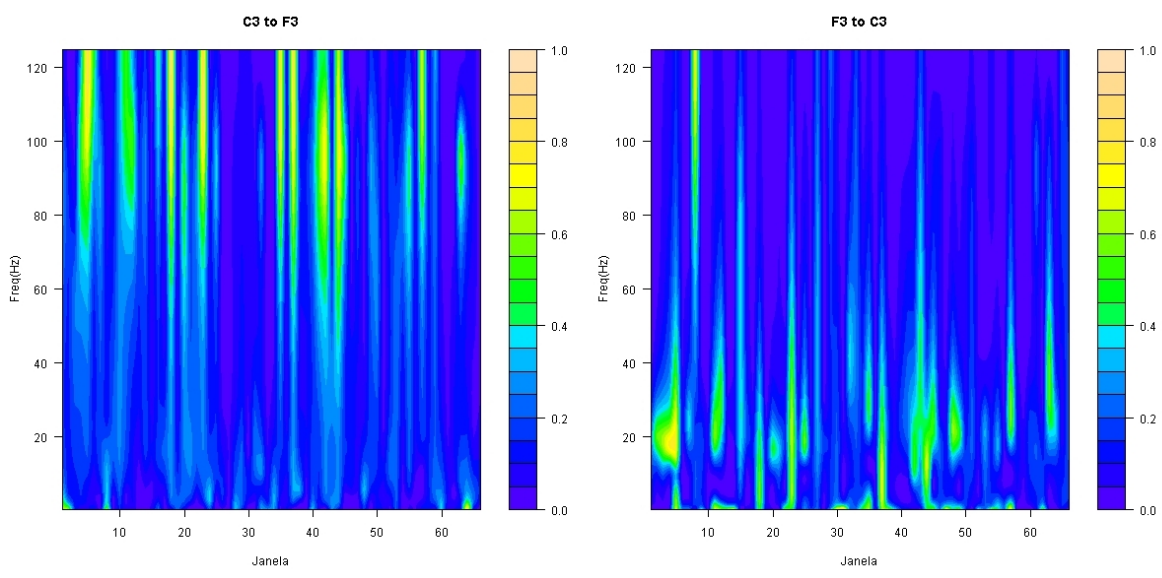

Figura B.14: Coerência parcial direcionada com janela móvel entre os sinais C3 e F3
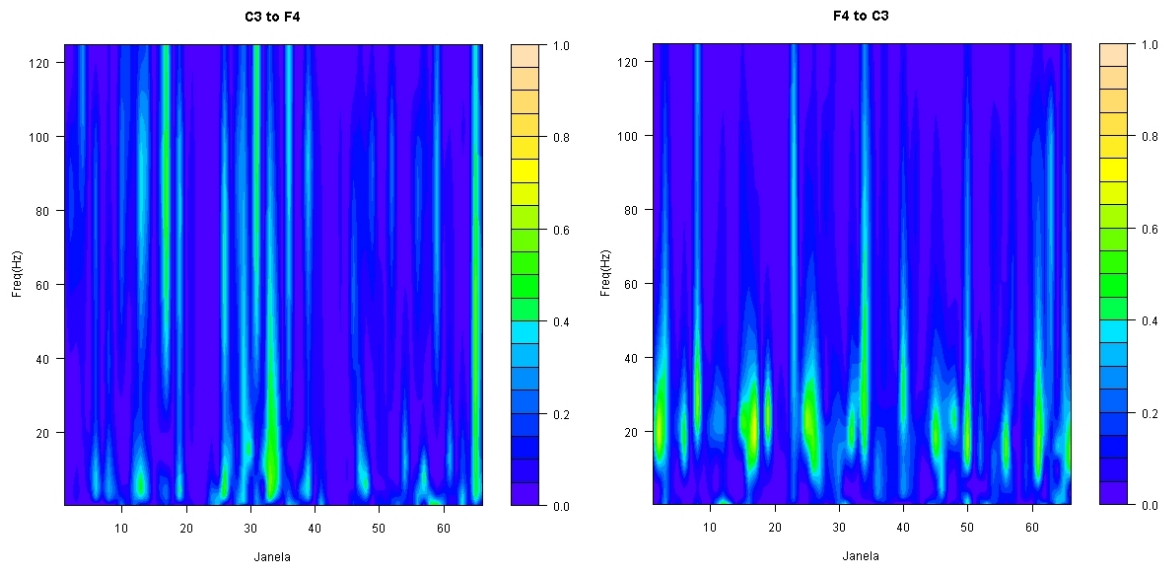

Figura B.15: Coerência parcial direcionada com janela móvel entre os sinais C3 e F4 

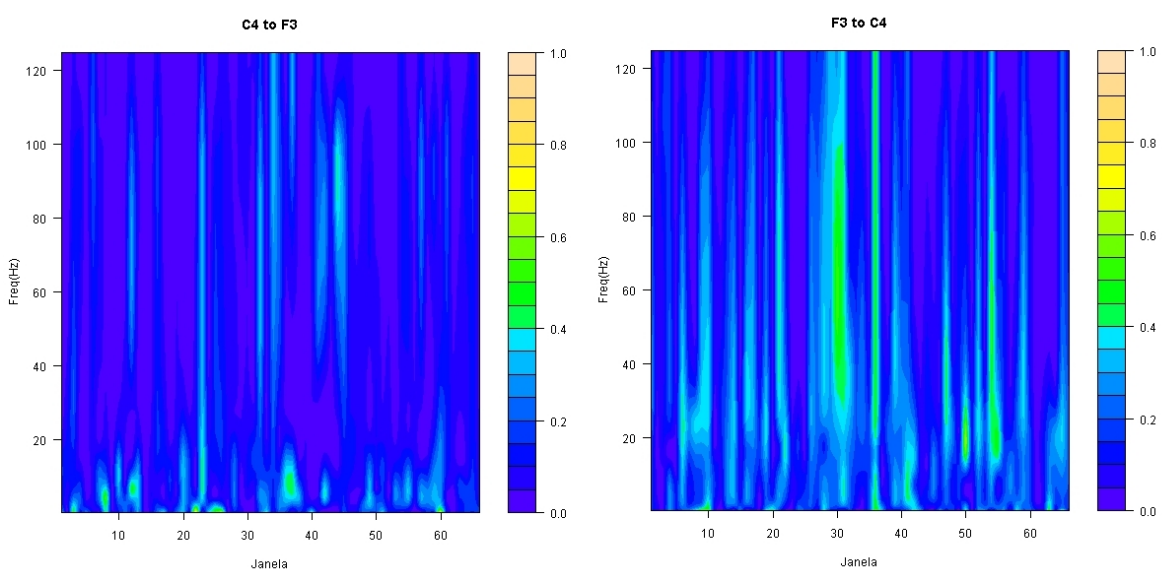

Figura B.16: Coerência parcial direcionada com janela móvel entre os sinais C4 e F3
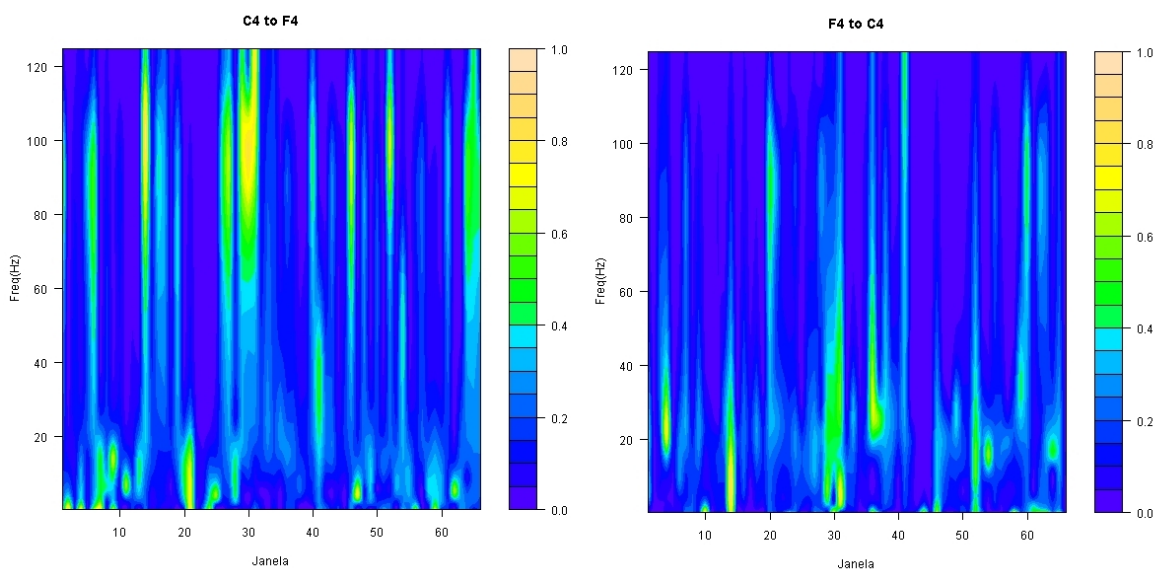

Figura B.17: Coerência parcial direcionada com janela móvel entre os sinais C4 e F4
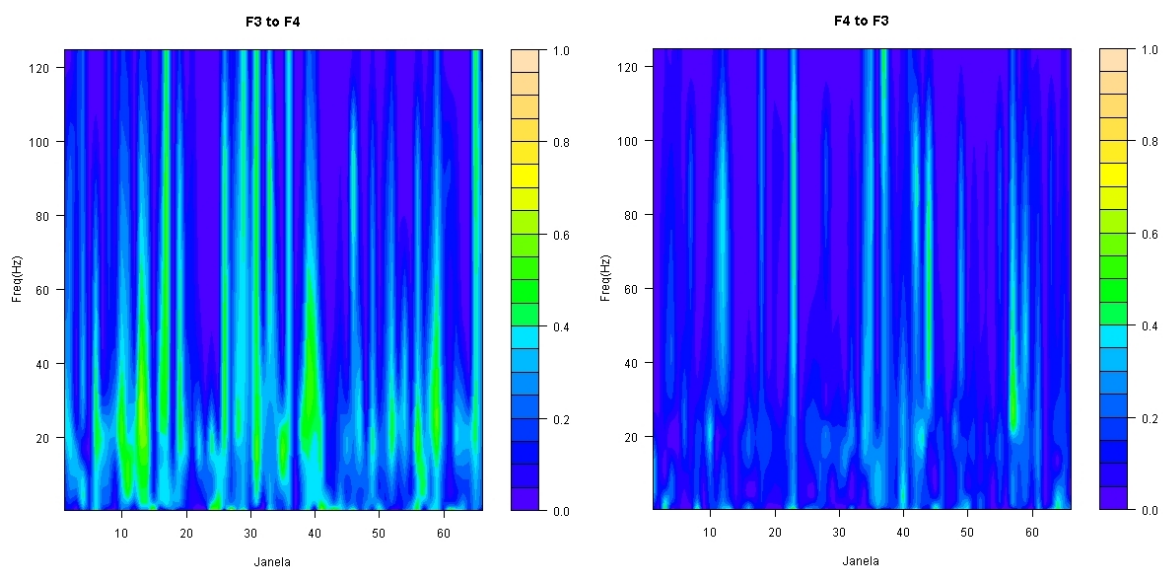

Figura B.18: Coerência parcial direcionada com janela móvel entre os sinais F3 e F4 

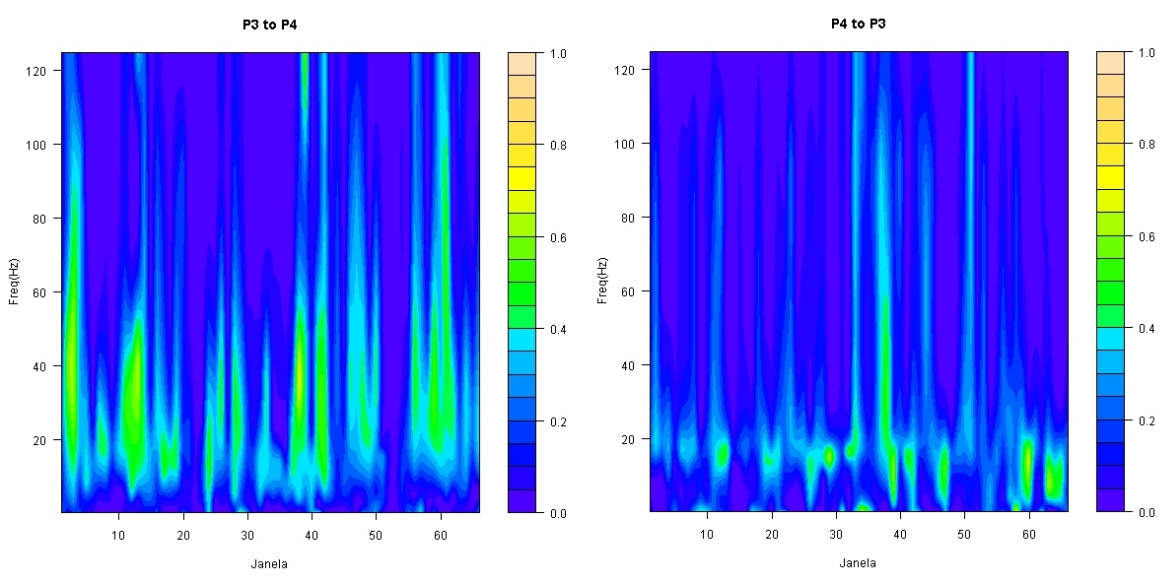

Figura B.19: Coerência parcial direcionada com janela móvel entre os sinais P3 e P4
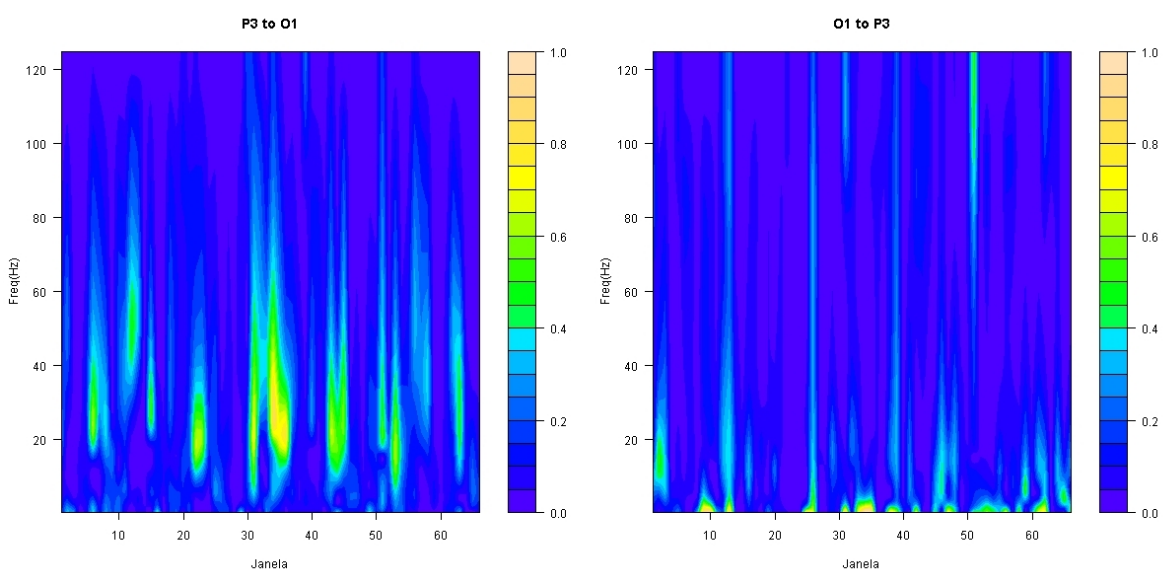

Figura B.20: Coerência parcial direcionada com janela móvel entre os sinais P3 e O1
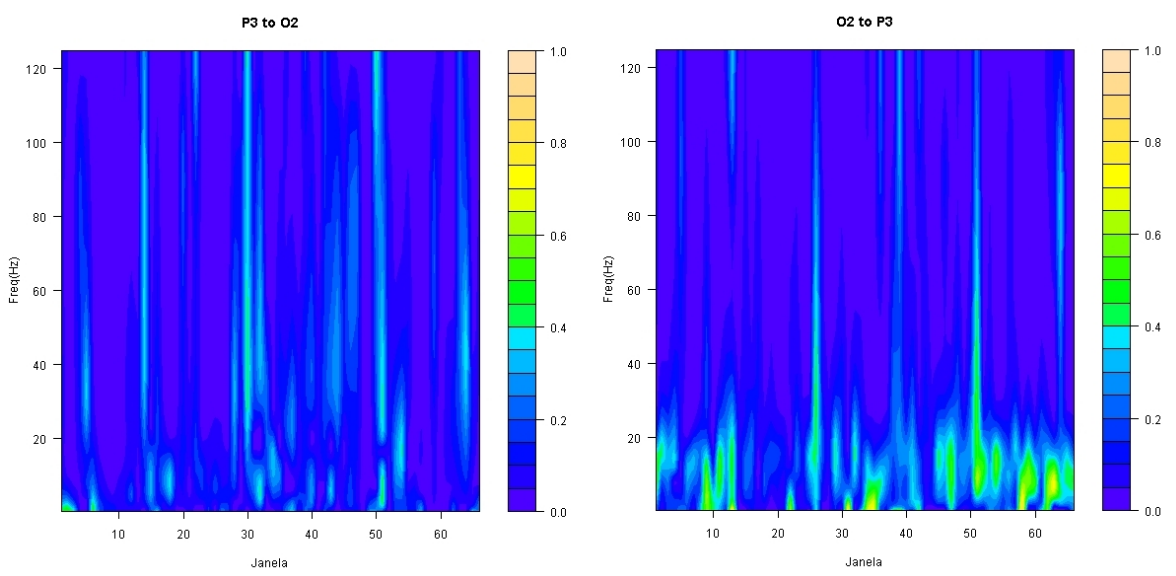

Figura B.21: Coerência parcial direcionada com janela móvel entre os sinais P3 e O2 

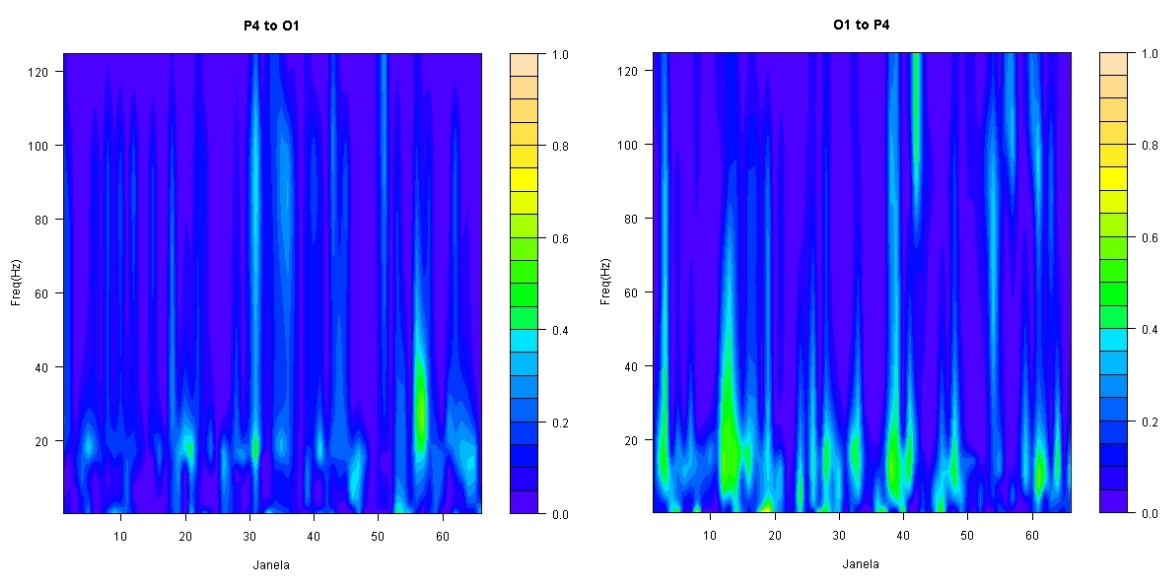

Figura B.22: Coerência parcial direcionada com janela móvel entre os sinais P4 e O1
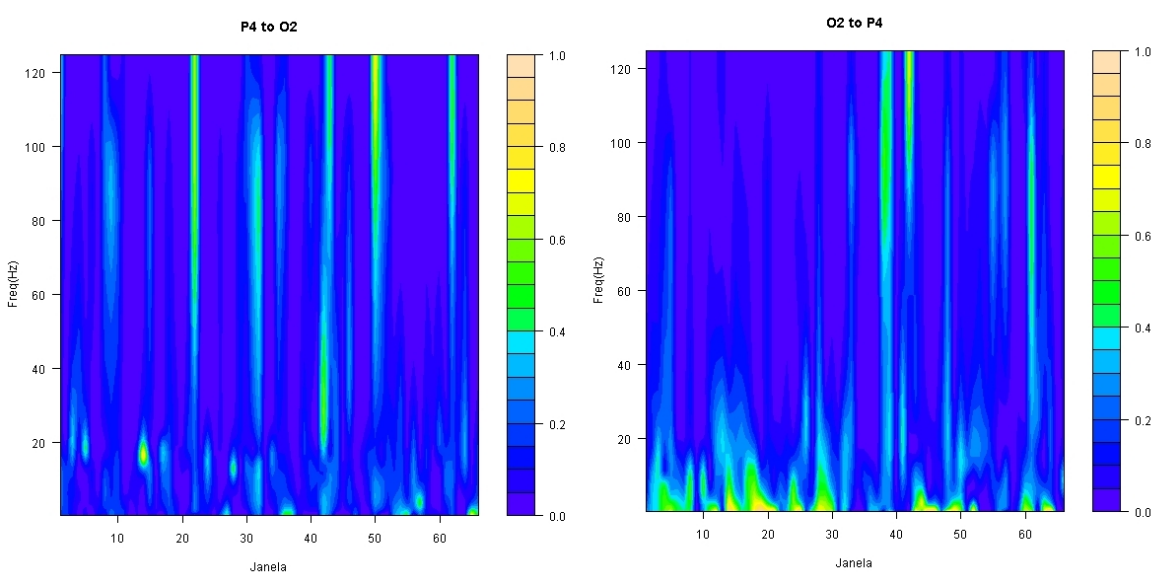

Figura B.23: Coerência parcial direcionada com janela móvel entre os sinais P4 e O2
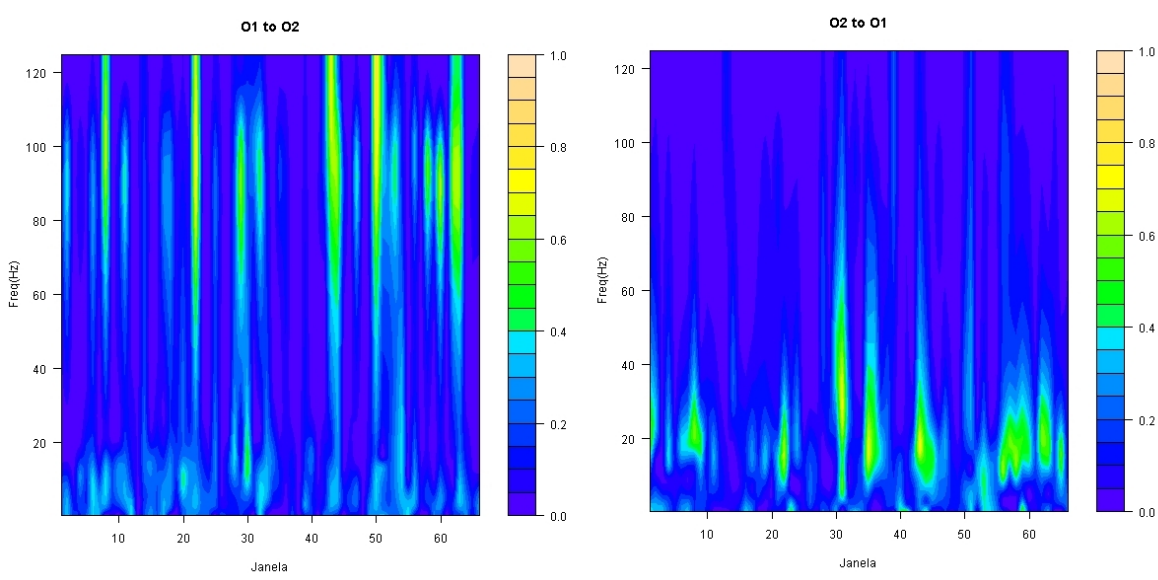

Figura B.24: Coerência parcial direcionada com janela móvel entre os sinais O1 e O2 

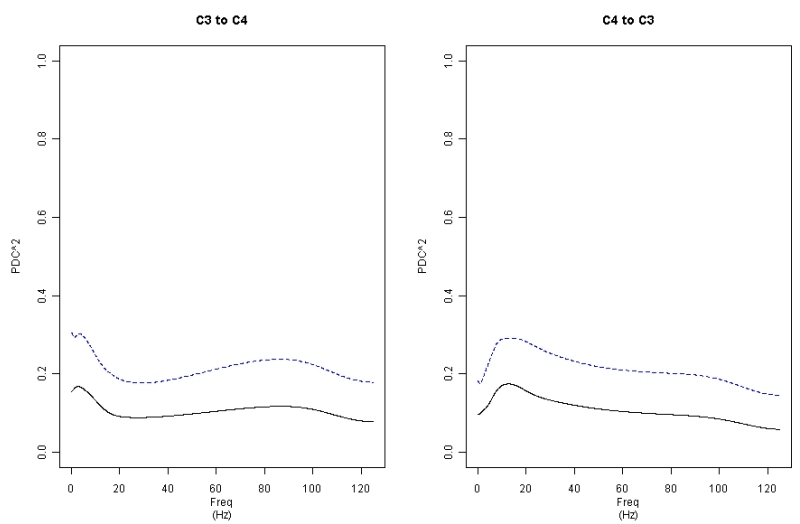

Figura B.25: Curva média das janelas móveis das PDCs entre os sinais C3 e C4. Em preto a curva da média e em azul(tracejado) o desvio-padrão.
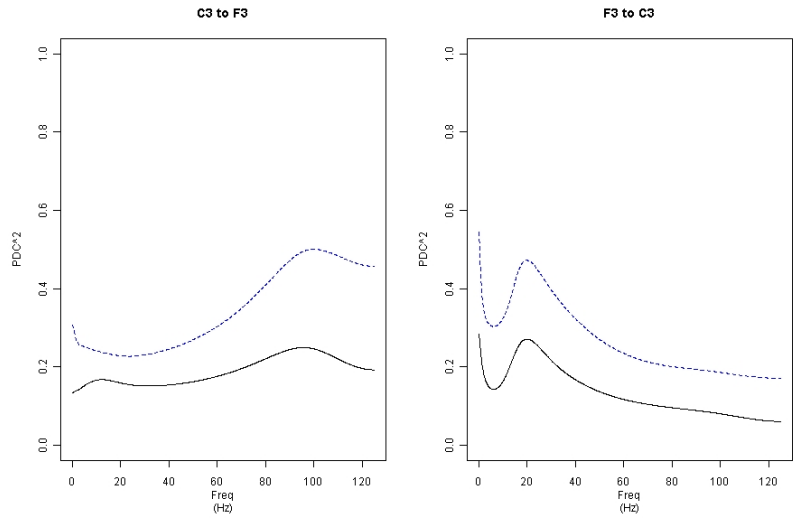

Figura B.26: Curva média das janelas móveis das PDCs entre os sinais C3 e F3. Em preto a curva da média e em azul(tracejado) o desvio-padrão.
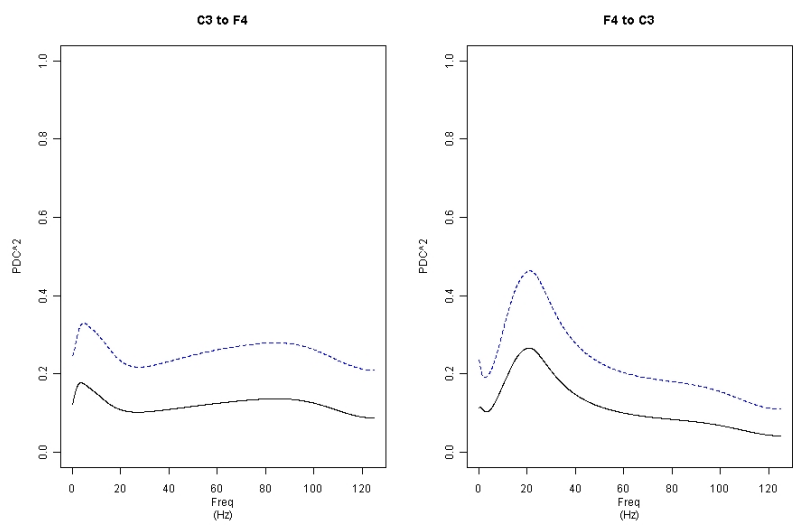

Figura B.27: Curva média das janelas móveis das PDCs entre os sinais C3 e F4. Em preto a curva da média e em azul(tracejado) o desvio-padrão. 

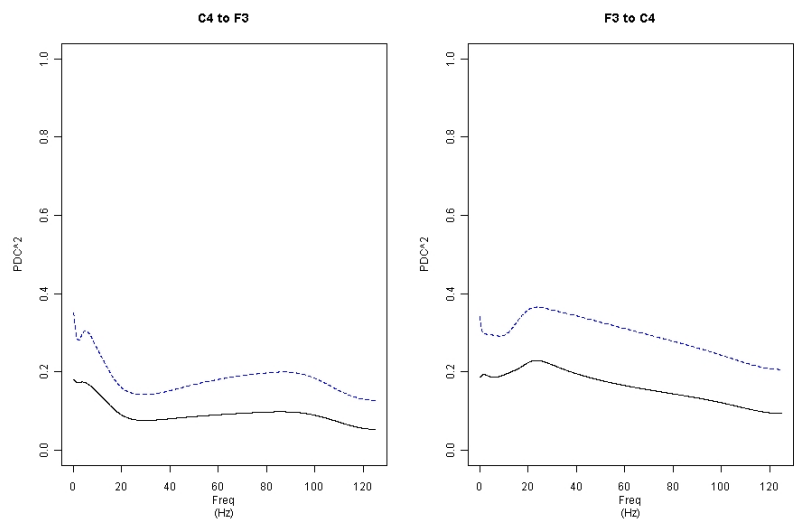

Figura B.28: Curva média das janelas móveis das PDCs entre os sinais C4 e F3. Em preto a curva da média e em azul(tracejado) o desvio-padrão.
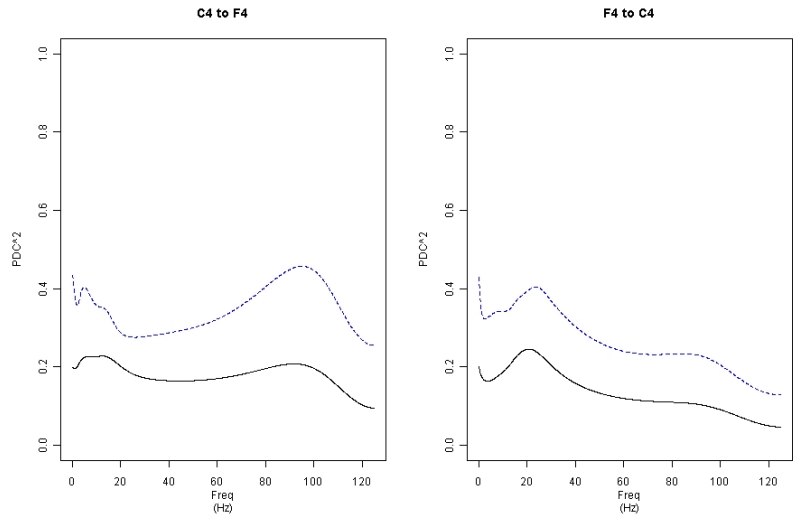

Figura B.29: Curva média das janelas móveis das PDCs entre os sinais C4 e F4. Em preto a curva da média e em azul(tracejado) o desvio-padrão.
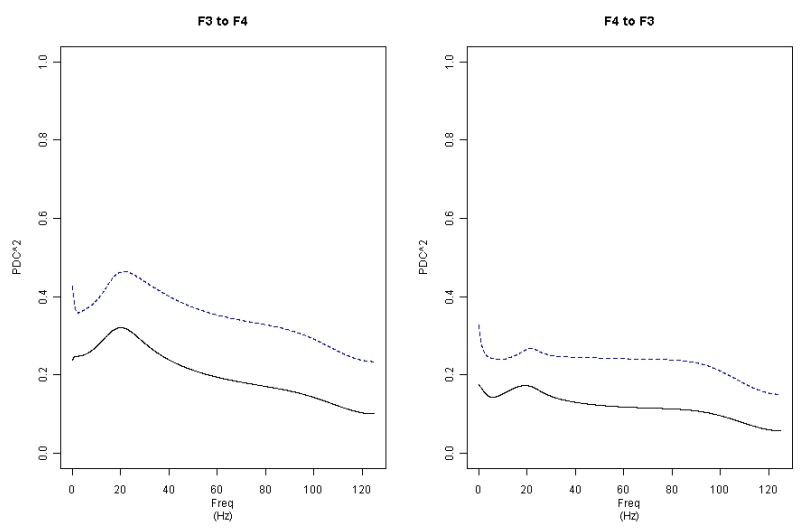

Figura B.30: Curva média das janelas móveis das PDCs entre os sinais F3 e F4. Em preto a curva da média e em azul(tracejado) o desvio-padrão. 

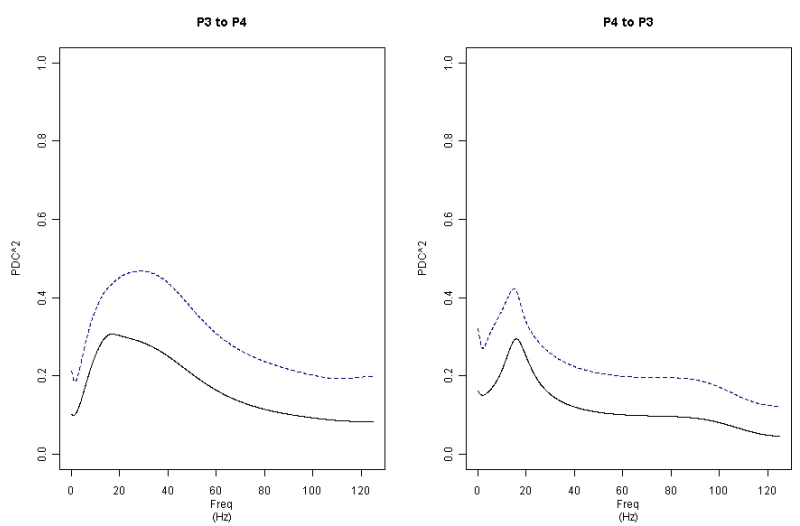

Figura B.31: Curva média das janelas móveis das PDCs entre os sinais P3 e P4. Em preto a curva da média e em azul(tracejado) o desvio-padrão.
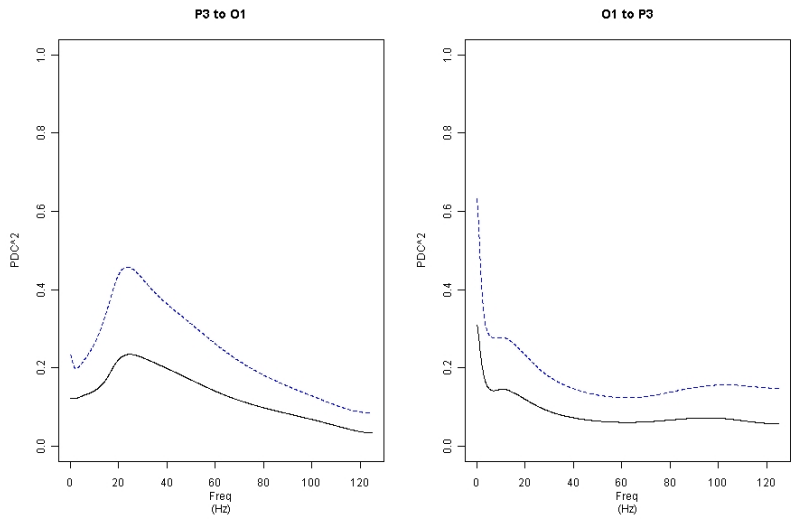

Figura B.32: Curva média das janelas móveis das PDCs entre os sinais P3 e O1. Em preto a curva da média e em azul(tracejado) o desvio-padrão.
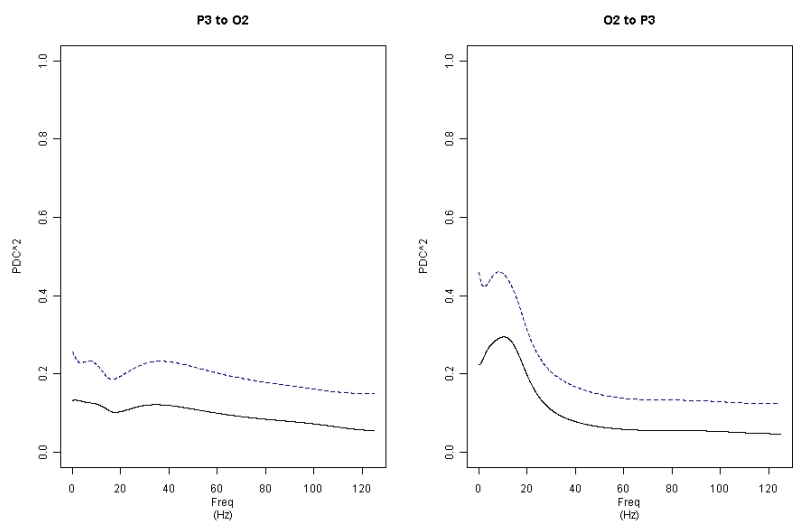

Figura B.33: Curva média das janelas móveis das PDCs entre os sinais P3 e O2. Em preto a curva da média e em azul(tracejado) o desvio-padrão. 

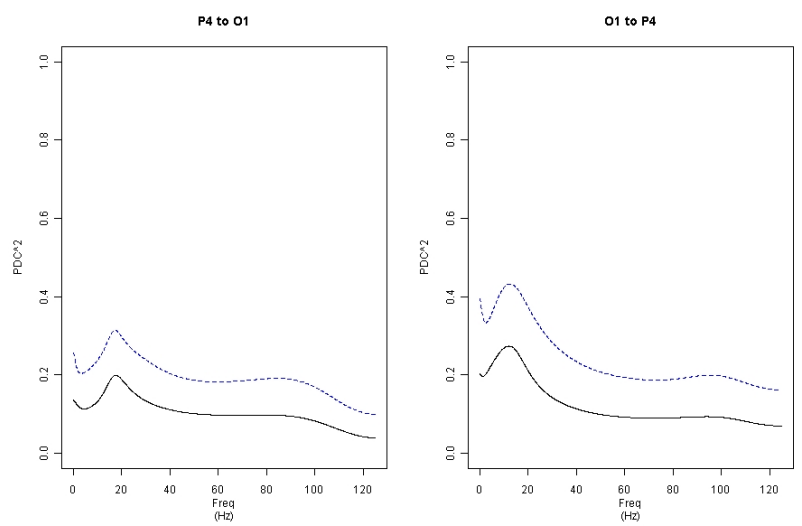

Figura B.34: Curva média das janelas móveis das PDCs entre os sinais P4 e O1. Em preto a curva da média e em azul(tracejado) o desvio-padrão.
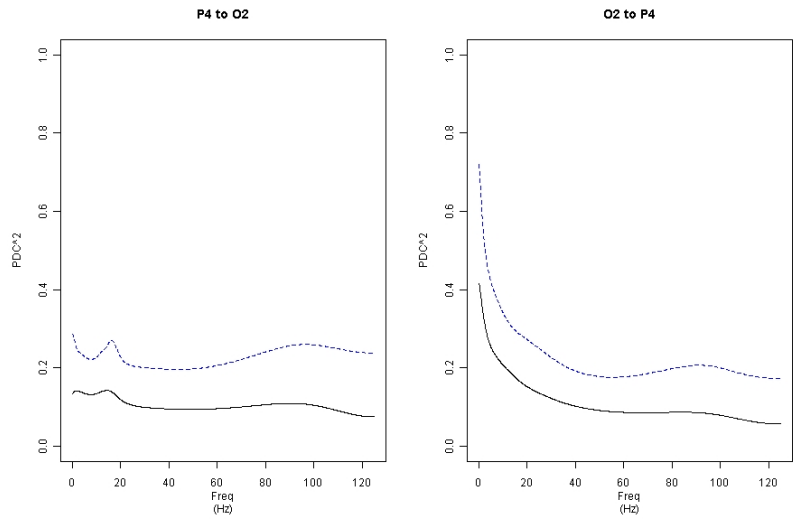

Figura B.35: Curva média das janelas móveis das PDCs entre os sinais P4 e O2. Em preto a curva da média e em azul(tracejado) o desvio-padrão.
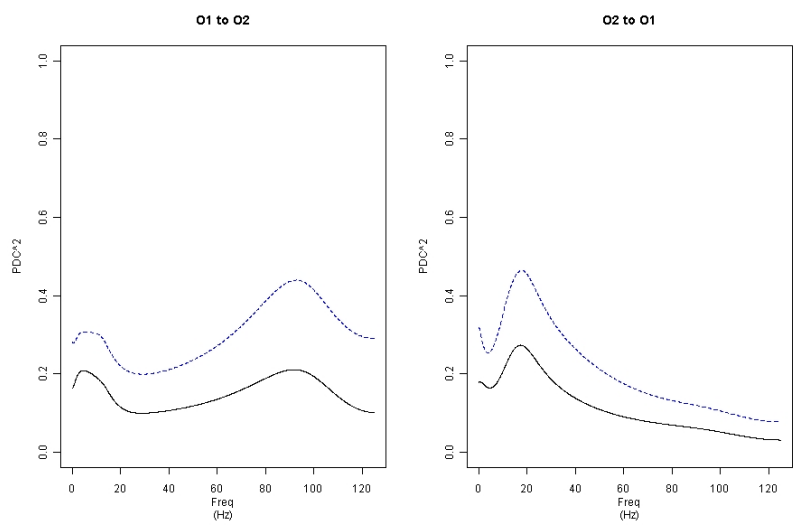

Figura B.36: Curva média das janelas móveis das PDCs entre os sinais O1 e O2. Em preto a curva da média e em azul(tracejado) o desvio-padrão. 


\section{Referências Bibliográficas}

[1] Anghinah, R. (2005). EEG Spectral Coherence, Rev Neurociencias, 13, p.50-53.

[2] Baccalá, L.A. and Sameshima, K. (2001). Partial Directed Coherence: A New Concept in Neural Structure Determination, Biological Cybernetics, 84, p.463-474.

[3] Baccalá, L.A., Sameshima, K. and Takahashi, D.Y. (2006). Computer Intensive Testing for Influence Between Time Series, Handbook of Time Series Analysis, 1.

[4] Barbosa, S.M.(2008). Pacote mAr obtido a partir de www.r-project.org.

[5] Biswal, B., Yetkin, F.Z., Haughton, V.M., Hyde, J.S. (1995). Functional Connectivity in the Motor Cortex of Resting Human Brain Using Echo-planar MRI, Magn Reson Med, 34, p.537541.

[6] Blinowska, K.J., Kus, R. and Kaminski, M. (2004). Granger Causality and Information Flow in Multivariate Processes, The American Phisical Society, Phisical Review, E 70.

[7] Blinowska, K.J., Kus, R. and Kaminski, M., Ginter Jr, J. (2008). Transmission of Brain Activity in Cognitive and Motor tasks, 30th Annual International IEEE EMBS conference, Phisical Review.

[8] Bloomfield, P. (2000). Fourier Analysis of Time Series: An Introduction, 2nd Ed., North Carolina: Wiley.

[9] Brillinger, D.R. (2001). Time Series: Data Analysis and Theory, expanded Ed., New York: Holt, Rinehart and Winston.

[10] Brockwell, P.J. and Davis, R.J. (1991). Time Series: Theory and Methods, 2nd Ed., New York: Springer-Verlag.

[11] Bruscato, A. (2000). Análise Espectral de Processos Não-Estacionários Utilizando a Transformada de Fourier, Dissertação de Mestrado, São Paulo: IME.

[12] Chatfield, C. (1995). The Analysis of Time Series: An Introduction, 5th Ed., London: Chapman \& Hall.

[13] Dahlhaus, R. (2000). Graphical Interaction Models for Multivariate Time Series, Metrika, 51, p.157-172. 
[14] Friston, K.J., Harrison, L. and Penny, W. (2003). Dynamic Causal Modelling, NeuroImage, 19, p.1273-1302.

[15] Ginter Jr., Blinowska, K.J., Kaminski, M., Durka, P.J., Pfurtscheller, G., Neuper, C. (2005). Propagation of EEG Activity in the Beta and Gamma Band During Movement Imagery in Humans, Methods inf Med, 44, p.106-113.

[16] Granger, C.W.J. (1969). Investigating Causal Relations By Econometric Models and CrossSpectral Methods, Econometrica, 37.

[17] Grinsted, A., Moore, J.C. and Jevrejeva, S. (2004). Application of the Cross Wavelet Transform and Wavelet Coherence to Geophysical Time Series, Nonlinear Processes in Geophysics, 11,p.561-566.

[18] van den Heuvel, M.P., Mandl, R.C.W., Kahn, R.S. and Hulshoff Pol, H.E. (2009). Functionally Linked Resting-State Networks Reflect the Underlying Structural Connectivity Architecture of the Human Brain, Humann Brain Mapping.

[19] Kaminski, M. and Blinowska, K.J. (1991). A New Method of the Description of the Information Flow, Biological Cybernetics, 65, p.203-210.

[20] Kaminski, M., Ding, M., Truccolo, W.A. and Bressler, S.T. (2001). Evaluating Casual Relations in Neural Systems: Granger Causality, Directed Transfer Function and Statistical Assessment of Significance, Biological Cybernetics, 85, p.145-157.

[21] Kaminski, M. (2005). Determination os Transmission Patterns in Multichannel Data, Philosophical Transactions os Royal Society, 360, p.947-952.

[22] Kaminski, M., Zygierewicz, J., Kus, R. and Crone, N. (2005). Analysis of Multichannel Biomedical Data, Neurobiologiae Experimentals, 65, p.443-452.

[23] Kaplan, A.Ya., Shishkin, S.L. (2000). Application of the Change-point Analysis to the Investigation of the Brain's Electrical Activity, Nonparametric Statistical Diagnosis: Problems and Methods, p.333-388.

[24] Kus, R., Kaminski, M. and Blinowska, K.J. (2004). Determination os EEG Activity Propagation: Pair-wise Versus Multichannel Estimate, IEEE Transactions on Biomedical Enginner Ing, 51.

[25] Di Martino, A., Scheres, A., Margulies, D.S., Kelly, A.M.C., Uddin, L.Q., Shehzad, Z., Biswal, B., Walters, J.R., Castellanos, F.X., (2008). Functional Connectivity of Human Striatum: a Resting State fMRI Study, Cerebral Cortex.18, p.2735-2747.

[26] Morettin, P.A. (2008). Econometria Financeira - Um Curso de Séries Temporais Financeiras, São Paulo: Ei13 Edgard Blücher. 
[27] Morettin, P.A. e Toloi, C.M.C. (2006). Análise de Séries Temporais, 2a Ed., São Paulo: Edgard Blücher.

[28] Park, D.C. and Lorenz, P.R.(2009). The Adaptative Brain: Aging and Neurocognitive Scaffolding, Annual Reviews of Psychology, 60, p.173-196.

[29] Percival, D.B. and Walden, A.T. (2000). Wavelet Methods for Time Series Analysis, New York: Cambridge University Press.

[30] Percival, D.B. and Walden, A.T. (1993). Spectral Analysis for Physical Applications: Multitaper and Conventional Univariate Techniques, New York: Cambridge University Press.

[31] Pinel, P., Thirion, B., Meriaux, S., Jobert, A. Serres, J., LeBihan, D., Poline, J.B. and Dehaene, S. (2007). Fast Reproducible Identification and Large-Scale Databasing of Individuals Functional Cognitive Networks, BMC Neuroscience, 8, p.91-.

[32] Priestley, M.B. (1981). Spectral Analysis and Time Series, 1 and 2, London: Academic Press.

[33] Saito, Y. and Harashima, H. (1981). Tracking of Information Within Multichannel EEG Record - Causal Analysis in EEG, Recent Advantages in EEG and EMG Data Processing, p.133-146.

[34] Sato, J.R., Takahashi, D.Y., Arcuri, S.M., Baccalá, L.A., Morettin, P.A. and Sameshima, K. (2009). Frequency Domain Connectivity Identification: An Application of Partial Directed Coherence in fMRI, Human Brain Mapping. 30, p.452-461.

[35] Sheline, Y.I., Barch, D.M., Price, J.L., Rundle, M.M., Vaishnavi, S.N., Snyder, A.Z., Mintun, M.A., Wang, S., Coalson, R.S., Raichle, M.E.(200o). The Default Mode Network and SelfReferential Processes in Depression, Proceedings of the National Academy of Sciences of the United States of America. 106, no.6, p.1942-1947.

[36] Shehzad, Z., Kelly, A.M.C., Reiss, P.T., Gotimer, K., Uddin, L.Q., Lee, S.H., Margulies, D.S. Amy, K.R., Biswal, B.B., Petkova, E., Castellanos, F.X., Milham, M. (2009). The Resting Brain: Unconstrained yet Reliable, Cerebral Cortex.

[37] Shumway, R.H. and Stoffer, D.S. (2006). Time Series Analysis and Its Applications, 2nd Ed., New York: Springer-Verlag.

[38] Sueur, J., Aubin, T. and Simonis, C. (2009). Pacote seewave obtido a partir de www.r-project.org.

[39] Takahashi, D.Y. ; Baccalá, L.A., Sameshima, K. (2007). Connectivity Inference via Partial Directed Coherence: Asymptotic Results, Journal of Applied Statistics, 34, p.1259-1273.

[40] Takahashi, D.Y. ; Baccalá, L.A., Sameshima, K. (2007a). On Partial Directed Coherence Factor, Granger Causality and Information Theory, 10th Tamagawa-Riken Brain Dynamics Brain Forum - DBF07. 
[41] Thirion, B., Dodel, S. and Poline, J.B. (2005). Detection of Signal Synchronizations in RestingState fMRI datasets, NeuroImage, 29, p.321-327.

[42] Torrence, C. and Webster, P.J. (1999). Interdecadal Changes in the ENSO - Monsoon System, Journal of Climate, 12, p.2679-2690.

[43] Torrence, C. and Compo, G.P. (1998). A practical Guide to Wavelet Analysis, Bulletin of the American Meteorological Society, 79, p.61-78.

[44] Uddin, L.Q., Kelly, A.M.C., Biswal, B.B., Castellanos, F.X. and Milham, M.P.(2008). Functional Connectivity of Default Mode Network Components: Correlation, Anticorrelation and Causality, Human Brain Mapping.

[45] Yamashita, O., Sadato, N., Okada, T. and Ozaki, T. (2005). Evaluating Frequency-Wise Directed Connectivity of BOLD Signals Applying Relative Power Contribution With Linear Multivariate Time-Series Models, NeuroImage, 25, p.478-490.

[46] Zheng, X. and Rajapakse, J.C. (2006). Learning Functional Structure from fMR Images, NeuroImage. 